\title{
Inverse scattering theory for discrete Schrödinger operators on the hexagonal lattice
}

\author{
Kazunori Ando \\ Graduate school of pure and applied science, University of Tsukuba \\ Tennoudai, Tsukuba, Ibaraki 305-8571, Japan \\ email: ando@math.tsukuba.ac.jp
}

November 20, 2018

\begin{abstract}
We consider the spectral theory for discrete Schrödinger operators on the hexagonal lattice and their inverse scattering problem. We give a procedure for reconstructing the compactly supported potential from the scattering matrix for all energies. The same procedure is applicable for the inverse scattering problem on the triangle lattice.
\end{abstract}

2000 Mathematics Subject Classification: 35P25, 39A12, 47B39, 81U40

\section{Introduction}

Let us begin with recalling the study of inverse scattering problems for Schrödinger operators on $\mathbb{R}^{n}$. For $n=1$, it was solved by Gel'fand and Levitan [7, Marčhenko [17] in 1950's. For $n=3$, Faddeev [5] established the uniqueness of the potential with given scattering matrix by using high-energy Born approximation soon after that. In the 1970's, Faddeev [6] and Newton [20] investigated an analogue of Gel'fand-Levitan theory for the multidimensional case by making use of Faddeev's Green operator. In the late 1980's, there was a breakthrough, which is called $\bar{\partial}$-approach, in this direction by Beals and Coifman [1, Nachman and Ablowitz [19, Khenkin and Novikov 21], Weder [25]. See e.g. a survey article of Isozaki [1].

In discrete settings, it was pointed out early that the theory of Gel'fandLevitan-Marčhenko is applicable for the discrete Schrödinger operator on $\mathbb{Z}$ (e.g. Case and Kac [2]). On $\mathbb{Z}^{n}, n \geq 2$, Isozaki and Korotyaev [9] studied the inverse scattering problem for the discrete Schrödinger operator with a compactly supported potential, recently. They used a kind of complex Born approximation, and derived a procedure for reconstructing the potential. Furthermore, they got a partial result about the inverse scattering problem from a fixed energy by using a discrete analogue of Faddeev's Green operator. 
In this paper, we consider the inverse scattering problem for the discrete Schrödinger operator on the hexagonal lattice, and derive a reconstruction procedure for the potential from the scattering matrix of all energies. The hexagonal lattice is a sort of two dimensional lattice, which covers the plane by equilateral hexagons with honeycomb structure. We regard the hexagonal lattice as graph. Before stating our main theorem, let us briefly recall the graph theory.

We denote by $G=(V(G), E(G))$ the graph that consists of a vertex set $V(G)$, whose cardinality is at most countable, and an edge set $E(G)$, each element of which connects a pair of vertices. Let $v, u \in V(G)$ and $e \in E(G)$. We denote by $v \stackrel{e}{\sim} u$, or simply $v \sim u$, when $v$ is adjacent to $u$ by $e$; by $N_{v}=\{u \in V(G) ; v \sim u\}$ the set of vertices which are adjacent to $v$. We also denote by $\operatorname{deg}(v)=\sharp N_{v}$ the degree of $v$. We assume that the graph $G$ is connected, which implies that $\operatorname{deg}(v)>0$ for any $v \in V(G)$, locally finite, that is, $\operatorname{deg}(v)<\infty$ for any $v \in V(G)$, and simple, that is, there are neither self-loops nor multiple edges. Here, a self-loop is an edge which joins a vertex to itself, and multiple edges are two or more edges which join the same two vertices. The discrete Laplacian $\Delta_{d}$ on $G$ is defined as

$$
\left(\Delta_{d} \hat{f}\right)(v)=\frac{1}{\operatorname{deg}(v)} \sum_{u \in N_{v}} \hat{f}(u)-\hat{f}(v)
$$

for the function $\hat{f}$ on $V(G)$. It is well-known that $-\Delta_{d}$ is a bounded, self-adjoint operator on

$$
l^{2}(G)=\left\{\hat{f}: V(G) \rightarrow \mathbb{C} ;\|\hat{f}\|_{l^{2}(G)}^{2}=\sum_{v \in V(G)}|\hat{f}(v)|^{2} \operatorname{deg}(v)<\infty\right\},
$$

and $\sigma\left(-\Delta_{d}\right) \subset[0,2]$. See e.g. Chung [4].

Let $G$ be the hexagonal lattice. Figure 1 illustrates the hexagonal lattice, where the vertices are represented as big black and white dots and the edges as segments between two of them. For simplicity's sake, we adopt as free Hamil-

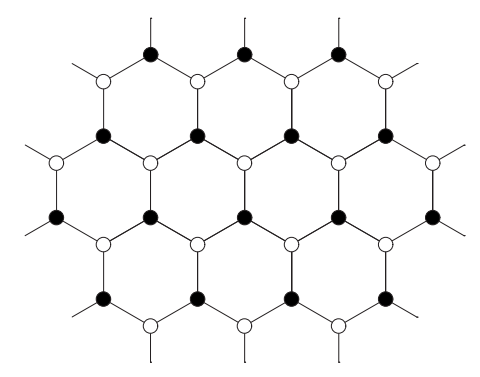

Figure 1: the hexagonal lattice as graph

tonian the operator which is unitarily equivalent to $3\left(\Delta_{d}+1\right)$ on the hexagonal 
lattice. See Section 2 for the details. The discrete Schrödinger operator is defined as the free Hamiltonian plus the potential $\hat{q}$, which is a multiplication operator by a real-valued function: $(\hat{q} \hat{f})(v)=\hat{q}(v) \hat{f}(v)$. Our main result is

Theorem 1.1. Assume that $\hat{q}(v)=0$ except for a finite number of $v \in V(G)$. Then from the scattering amplitude $A(\lambda)$ for all $\lambda \in \sigma_{\text {ess }}(H)$, one can uniquely compute $(\hat{q}(v))_{v \in V(G)}$.

The hexagonal lattice can be viewed as a discrete model of graphene, which is a two dimensional, single-layered carbon sheet with honeycomb structure. Therefore, discrete Schrödinger operators on the hexagonal lattice are regarded as discrete Hamiltonians on graphene. In physics, graphene is one of the most interesting subjects due to the peculiar behavior of electrons, and very actively studied, recently. Discrete Schrödinger operators, or tight binding models of Hamiltonians, are widely used to investigate graphene (Neto, Guinea, Peres, Novoselov and Geim [3]). Another approach is to study quantum graphs (Kuchment and Post [14]).

If we introduce a small parameter and take the limit to zero around the Dirac points, it is well-known that the discrete Schrödinger operator on the hexagonal lattice tends to the Dirac operator on $\mathbb{R}^{2}$ (Semenoff [24, González, Guinea and Vozmediano [8]). Therefore, in a sense, we are considering a discrete version of the inverse scattering problem for Dirac operators in two dimensions. It is not known so much about the inverse scattering theory for Dirac operators, relative to that for Schrödinger operators. We refer to Isozaki [10.

The rest of this paper is organized as follows: In Section 2, we review spectral properties of the free Hamiltonian on the hexagonal lattice, and define a conjugate operator to derive Mourre estimates. As a consequence, we show the absolute continuity of the discrete Schrödinger operator on the hexagonal lattice. In Section 3, we construct a spectral representation, and then obtain a representation of the S-matrix. Our main result, the reconstruction procedure for the potential, is shown in Section 6, and the key lemmas for it, analytic continuation and estimates of the resolvent, are proved in Sections 4 and 5, respectively. Section 7 remarks that our reconstruction procedure also works on the triangle lattice in almost the same way as on the hexagonal lattice and the two-dimensional square lattice. Some technical lemmas are proved in Appendices.

\section{Discrete Schrödinger operators on the hexag- onal lattice}

\subsection{Preliminaries}

As far as the discrete Laplacian is concerned, we only have to take care of the adjacent relations between the vertices, which enables us to illustrate the hexagonal lattice $G$ as Figure2, where the vertices are placed on $\mathbb{Z}^{2}$. Therefore, 


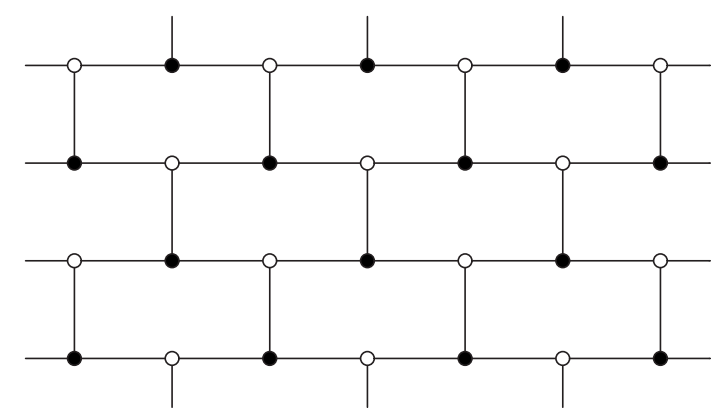

Figure 2: The hexagonal lattice can be deformed as above, where the black and white dots are placed on $\mathbb{Z}^{2}$, and correspond to those in Figure1, respectively.

we can regard that

$$
\begin{gathered}
V(G)=\mathbb{Z}^{2}, \\
E(G)=\left\{[m, n] ; m, n \in \mathbb{Z}^{2}, n_{1}=m_{1}+1, m_{2}=n_{2}\right\} \\
\cup\left\{[m, n] ; m, n \in \mathbb{Z}^{2}, m_{1}=n_{1}, m_{2} \in 2 \mathbb{Z}+1, n_{2}=m_{2}+1\right\} \\
\cup\left\{[m, n] ; m, n \in \mathbb{Z}^{2}, m_{1}=n_{1}, m_{2} \in 2 \mathbb{Z}, n_{2}=m_{2}+1\right\},
\end{gathered}
$$

where $m=\left(m_{1}, m_{2}\right)$ and $n=\left(n_{1}, n_{2}\right)$, and the segment between $m$ and $n$ is denoted by $[m, n]$. Also, noticing that the degree of the vertices on the hexagonal lattice is always three, we can regard $l^{2}(G)$ as just the $l^{2}$-space on $\mathbb{Z}^{2}$ equipped with the norm $\|\hat{g}\|_{l^{2}(G)}^{2}=3 \sum_{n \in \mathbb{Z}^{2}}|\hat{g}(n)|^{2}$.

We next introduce the hexagonal lattice structure on $\mathbb{Z}^{2}$, which is different from the standard lattice one, in the following manner. We split $\mathbb{Z}^{2}$ into two parts: $\mathbb{Z}^{2}=\mathbb{Z}_{e}^{2} \cup \mathbb{Z}_{o}^{2}$, where

$$
\begin{gathered}
\mathbb{Z}_{e}^{2}=\left\{\left(n_{1}, n_{2}\right) ; n_{1}+n_{2} \in 2 \mathbb{Z}\right\}, \\
\mathbb{Z}_{o}^{2}=\left\{\left(n_{1}, n_{2}\right) ; n_{1}+n_{2}-1 \in 2 \mathbb{Z}\right\} .
\end{gathered}
$$

In Figure 2, $\mathbb{Z}_{e}^{2}$ and $\mathbb{Z}_{o}^{2}$ are represented as the black dots and the white ones, respectively, each of which has a lattice structure with basis $\vec{e}_{1}=(1,1)$ and $\vec{e}_{2}=(-1,1)$. Therefore, there exists a canonical isomorphisms

$$
\begin{gathered}
\mathbb{Z}_{e}^{2} \ni\left(n_{1}, n_{2}\right) \longmapsto\left(m_{1}, m_{2}\right) \in \mathbb{Z}^{2}, \\
n_{1}=m_{1}-m_{2}, n_{2}=m_{1}+m_{2},
\end{gathered}
$$

and

$$
\begin{gathered}
\mathbb{Z}_{o}^{2} \ni\left(n_{1}, n_{2}\right) \longmapsto\left(m_{1}, m_{2}\right) \in \mathbb{Z}^{2}, \\
n_{1}=m_{1}-m_{2}, n_{2}=1+m_{1}+m_{2} .
\end{gathered}
$$

Noticing that $\mathbb{Z}_{e}^{2}$ and $\mathbb{Z}_{o}^{2}$ are the minimal periodic lattice structures in the hexagonal lattice, we need to use a system of difference operators to analyze the discrete Laplacian on the hexagonal lattice. 


\subsection{Discrete Laplacian}

There is a natural unitary mapping $\mathcal{J}:\left(l^{2}\left(\mathbb{Z}^{2}\right)\right)^{2} \rightarrow l^{2}(G)$, more precisely,

$$
\begin{gathered}
\mathcal{J}: l^{2}\left(\mathbb{Z}^{2}\right) \oplus l^{2}\left(\mathbb{Z}^{2}\right) \ni \hat{f}=\left(\hat{f}_{1}, \hat{f}_{2}\right) \longmapsto \hat{g} \in l^{2}(G), \\
\hat{g}\left(n_{1}, n_{2}\right)=(\mathcal{J} \hat{f})\left(n_{1}, n_{2}\right)= \begin{cases}\frac{1}{\sqrt{3}} \hat{f}_{1}\left(m_{1}, m_{2}\right), & \text { if }\left(n_{1}, n_{2}\right) \in \mathbb{Z}_{e}^{2}, \\
\frac{1}{\sqrt{3}} \hat{f}_{2}\left(m_{1}, m_{2}\right), & \text { if }\left(n_{1}, n_{2}\right) \in \mathbb{Z}_{o}^{2},\end{cases}
\end{gathered}
$$

where $\left(m_{1}, m_{2}\right)$ is defined by (11) and (2), respectively. Note that $\sqrt{3}$ comes from the degree of the vertices, which contributes to the definition of the norm of $l^{2}(G)$.

The adjacent vertices of $\left(n_{1}, n_{2}\right) \in \mathbb{Z}_{e}^{2}$ are $\left(n_{1} \pm 1, n_{2}\right),\left(n_{1}, n_{2}+1\right)$, and those of $\mathbb{Z}_{o}^{2}$ are $\left(n_{1} \pm 1, n_{2}\right),\left(n_{1}, n_{2}-1\right)$. Therefore, $3\left(\Delta_{d}+1\right)$ on $G$ is written as

$$
\begin{aligned}
& \left(3\left(\Delta_{d}+1\right) \hat{g}\right)\left(n_{1}, n_{2}\right) \\
= & \begin{cases}\hat{g}\left(n_{1}, n_{2}+1\right)+\hat{g}\left(n_{1}-1, n_{2}\right)+\hat{g}\left(n_{1}+1, n_{2}\right), & \text { if }\left(n_{1}, n_{2}\right) \in \mathbb{Z}_{e}^{2}, \\
\hat{g}\left(n_{1}, n_{2}-1\right)+\hat{g}\left(n_{1}-1, n_{2}\right)+\hat{g}\left(n_{1}+1, n_{2}\right), & \text { if }\left(n_{1}, n_{2}\right) \in \mathbb{Z}_{o}^{2},\end{cases}
\end{aligned}
$$

for $\hat{g} \in l^{2}(G)$. This implies that

$$
\begin{aligned}
& \left(\mathcal{J}^{*} 3\left(\Delta_{d}+1\right) \mathcal{J} \hat{f}\right)_{1}\left(m_{1}, m_{2}\right)=\hat{f}_{2}\left(m_{1}, m_{2}\right)+\hat{f}_{2}\left(m_{1}-1, m_{2}\right)+\hat{f}_{2}\left(m_{1}, m_{2}-1\right), \\
& \left(\mathcal{J}^{*} 3\left(\Delta_{d}+1\right) \mathcal{J} \hat{f}\right)_{2}\left(m_{1}, m_{2}\right)=\hat{f}_{1}\left(m_{1}, m_{2}\right)+\hat{f}_{1}\left(m_{1}+1, m_{2}\right)+\hat{f}_{1}\left(m_{1}, m_{2}+1\right),
\end{aligned}
$$

for $\hat{f}=\left(\hat{f}_{1}, \hat{f}_{2}\right) \in l^{2}\left(\mathbb{Z}^{2}\right) \oplus l^{2}\left(\mathbb{Z}^{2}\right)$.

Let us define

$$
l^{2}\left(\mathbb{Z}^{2} ; \mathbb{C}^{2}\right)=\left\{\hat{f}=\left(\left(\begin{array}{l}
\hat{f}_{1}(m) \\
\hat{f}_{2}(m)
\end{array}\right)\right)_{m \in \mathbb{Z}^{2}} ;\|\hat{f}\|_{l^{2}\left(\mathbb{Z}^{2} ; \mathbb{C}^{2}\right)}^{2}=\sum_{m \in \mathbb{Z}^{2}}\left(\left|\hat{f}_{1}(m)\right|^{2}+\left|\hat{f}_{2}(m)\right|^{2}\right)<\infty\right\} .
$$

There is a unitary mapping $\mathcal{I}: l^{2}\left(\mathbb{Z}^{2} ; \mathbb{C}^{2}\right) \rightarrow\left(l^{2}\left(\mathbb{Z}^{2}\right)\right)^{2}$, under which we can naturally identify $l^{2}\left(\mathbb{Z}^{2} ; \mathbb{C}^{2}\right)$ with $\left(l^{2}\left(\mathbb{Z}^{2}\right)\right)^{2}$, more precisely,

$\mathcal{I}: l^{2}\left(\mathbb{Z}^{2} ; \mathbb{C}^{2}\right) \ni\left(\left(\begin{array}{c}\hat{f}_{1}(m) \\ \hat{f}_{2}(m)\end{array}\right)\right)_{m \in \mathbb{Z}^{2}} \longmapsto\left(\left(\hat{f}_{1}(m)\right)_{m \in \mathbb{Z}^{2}},\left(\hat{f}_{2}(m)\right)_{m \in \mathbb{Z}^{2}}\right) \in l^{2}\left(\mathbb{Z}^{2}\right) \oplus l^{2}\left(\mathbb{Z}^{2}\right)$.

Let $\hat{H}_{0}=\mathcal{I}^{*} \mathcal{J}^{*} 3\left(\Delta_{d}+1\right) \mathcal{J} \mathcal{I}$. Then we can write

$$
\left(\hat{H}_{0} \hat{f}\right)(m)=\left(\begin{array}{l}
\hat{f}_{2}\left(m_{1}, m_{2}\right)+\hat{f}_{2}\left(m_{1}-1, m_{2}\right)+\hat{f}_{2}\left(m_{1}, m_{2}-1\right) \\
\hat{f}_{1}\left(m_{1}, m_{2}\right)+\hat{f}_{1}\left(m_{1}+1, m_{2}\right)+\hat{f}_{1}\left(m_{1}, m_{2}+1\right)
\end{array}\right)
$$

for

$$
\hat{f}=\left(\left(\begin{array}{l}
\hat{f}_{1}(m) \\
\hat{f}_{2}(m)
\end{array}\right)\right)_{m \in \mathbb{Z}^{2}} \in l^{2}\left(\mathbb{Z}^{2} ; \mathbb{C}^{2}\right) .
$$


We put $\mathbb{T}^{2}=\mathbb{R}^{2} /(2 \pi \mathbb{Z})^{2}$ and

$L^{2}\left(\mathbb{T}^{2} ; \mathbb{C}^{2}\right)=\left\{f=\left(f_{1}(\xi), f_{2}(\xi)\right) ;\|f\|_{L^{2}\left(\mathbb{T}^{2} ; \mathbb{C}^{2}\right)}^{2}=\int_{\mathbb{T}^{2}}\left(\left|f_{1}(\xi)\right|^{2}+\left|f_{2}(\xi)\right|^{2}\right) d \xi<\infty\right\}$.

We define a unitary operator $\mathcal{F}: l^{2}\left(\mathbb{Z}^{2}\right) \rightarrow L^{2}\left(\mathbb{T}^{2}\right)$ and its adjoint $\mathcal{F}^{*}$ by

$$
\begin{gathered}
(\mathcal{F} \hat{f})(\xi)=\frac{1}{2 \pi} \sum_{n \in \mathbb{Z}^{2}} \hat{f}(n) e^{i n \xi}, \\
\left(\mathcal{F}^{*} f\right)(n)=\frac{1}{2 \pi} \int_{\mathbb{T}^{2}} f(\xi) e^{-i n \xi} d \xi,
\end{gathered}
$$

and extend them naturally on $l^{2}\left(\mathbb{Z}^{2} ; \mathbb{C}^{2}\right)$ and $L^{2}\left(\mathbb{T}^{2} ; \mathbb{C}^{2}\right)$. They are the usual Fourier series and Fourier coefficients for each component. We put

$$
H_{0}=\mathcal{F} \hat{H}_{0} \mathcal{F}^{*} .
$$

Then $H_{0}$ is a multiplication operator by a symmetric matrix on $L^{2}\left(\mathbb{T}^{2} ; \mathbb{C}^{2}\right)$ :

$$
\left(H_{0} f\right)(\xi)=H_{0}(\xi) f(\xi),
$$

where

$$
\begin{gathered}
H_{0}(\xi)=\left(\begin{array}{cc}
0 & \alpha(\xi) \\
\bar{\alpha}(\xi) & 0
\end{array}\right), \\
\alpha(\xi)=1+e^{i \xi_{1}}+e^{i \xi_{2}}, \xi=\left(\xi_{1}, \xi_{2}\right) \in \mathbb{T}^{2}, \\
\bar{\alpha}(\xi)=\overline{\alpha(\xi)} .
\end{gathered}
$$

Next, let us define the unitary operator $\mathcal{U}$ as

$$
(\mathcal{U} f)(\xi)=U(\xi) f(\xi)
$$

on $L^{2}\left(\mathbb{T}^{2} ; \mathbb{C}^{2}\right)$, where

$$
U(\xi)=\frac{1}{\sqrt{2}}\left(\begin{array}{cc}
1 & \alpha(\xi) /|\alpha(\xi)| \\
1 & -\alpha(\xi) /|\alpha(\xi)|
\end{array}\right)
$$

is a unitary matrix for each $\xi \in \mathbb{T}^{2}$. Its adjoint $\mathcal{U}^{*}$ is a multiplication operator by the adjoint matrix $U^{*}(\xi)=U(\xi)^{*}$. We put

$$
\tilde{H}_{0}=\mathcal{U} H_{0} \mathcal{U}^{*} .
$$

The conjugation by $U(\xi)$ diagonalizes $H_{0}(\xi)$, which means that $\tilde{H}_{0}$ is a multiplication operator by a diagonal matrix on $L^{2}\left(\mathbb{T}^{2} ; \mathbb{C}^{2}\right)$ :

$$
\left(\tilde{H}_{0} f\right)(\xi)=\tilde{H}_{0}(\xi) f(\xi),
$$

where

$$
\tilde{H}_{0}(\xi)=U(\xi) H_{0}(\xi) U^{*}(\xi)=\left(\begin{array}{cc}
p(\xi) & 0 \\
0 & -p(\xi)
\end{array}\right)
$$




$$
p(\xi)=|\alpha(\xi)|=\sqrt{3+2 \cos \xi_{1}+2 \cos \xi_{2}+2 \cos \left(\xi_{1}-\xi_{2}\right)} .
$$

One computes that

$$
\begin{aligned}
& 3+2 \cos \xi_{1}+2 \cos \xi_{2}+2 \cos \left(\xi_{1}-\xi_{2}\right) \\
= & 4\left(\cos \frac{\xi_{1}-\xi_{2}}{2}+\frac{1}{2} \cos \frac{\xi_{1}+\xi_{2}}{2}\right)^{2}-\cos ^{2} \frac{\xi_{1}+\xi_{2}}{2}+1 .
\end{aligned}
$$

We put

$$
\xi_{0,1}=\left(\frac{2 \pi}{3},-\frac{2 \pi}{3}\right), \xi_{0,2}=\left(-\frac{2 \pi}{3}, \frac{2 \pi}{3}\right) \in \mathbb{T}^{2}
$$

Then we have

Proposition 2.1. $p(\xi)$ is continuous and

$$
0 \leq p(\xi) \leq 3
$$

on $\mathbb{T}^{2}$. Moreover,

1. $p(\xi)=0$ if and only if $\xi=\xi_{0,1}$ or $\xi_{0,2}$,

2. $p(\xi)=3$ if and only if $\xi=(0,0)$,

3. $p(\xi)$ is real-analytic on $\mathbb{T}^{2} \backslash\left\{\xi_{0,1}, \xi_{0,2}\right\}$.

Proof. Clearly, $p(\xi)^{2}$ is real-analytic and

$$
0 \leq p(\xi)^{2}=|\alpha(\xi)|^{2}=3+2 \cos \xi_{1}+2 \cos \xi_{2}+2 \cos \left(\xi_{1}-\xi_{2}\right) \leq 9
$$

on $\mathbb{T}^{2}$, which proves the first statement.

By using (6), we have $p(\xi)=0$ if and only if

$$
\begin{gathered}
\cos \frac{\xi_{1}-\xi_{2}}{2}+\frac{1}{2} \cos \frac{\xi_{1}+\xi_{2}}{2}=0, \\
\cos \frac{\xi_{1}+\xi_{2}}{2}= \pm 1,
\end{gathered}
$$

which means that the zeros of $p(\xi)$ are exactly $\xi_{0, j}, j \in\{1,2\}$.

The next statement is obvious.

Noticing that $\xi_{0,1}$ and $\xi_{0,2}$ are all the zeros of $p(\xi)^{2}$, we have the last one.

We denote by $\sigma(T)$ the spectrum of a self-adjoint operator $T$; by $\sigma_{a c}(T)$, $\sigma_{s c}(T)$, and $\sigma_{p p}(T)$ its absolutely continuous one, its singularly continuous one, and its pure point one, respectively. By using the above proposition, we have

Proposition 2.2. $\sigma\left(\tilde{H}_{0}\right)=\sigma_{a c}\left(\tilde{H}_{0}\right)=[-3,3]$ and $\sigma_{p p}\left(\tilde{H}_{0}\right)=\sigma_{s c}\left(\tilde{H}_{0}\right)=\emptyset$.

Remark 2.1. In the graph theory, the hexagonal lattice is, in general, called an abelian covering graph of a finite graph. For more general treatments for the spectrum of the discrete Laplacian on such a graph, we refer to Kotani, Shirai and Sunada [13]. 


\subsection{Discrete Schrödinger operators}

The potential on $l^{2}\left(\mathbb{Z}^{2} ; \mathbb{C}^{2}\right)$ is denoted by $\hat{q}$, which is a multiplication operator by real-valued, diagonal, $2 \times 2$ matrices:

$$
\hat{q}=\sum_{n \in \mathbb{Z}^{2}} \hat{q}(n) \hat{P}(n)
$$

where

$$
\begin{gathered}
\hat{q}(n)=\left(\begin{array}{cc}
\hat{q}_{1}(n) & 0 \\
0 & \hat{q}_{2}(n)
\end{array}\right), \\
\hat{q}_{j}(n) \in \mathbb{R}, j \in\{1,2\} .
\end{gathered}
$$

Here we denote by $\hat{P}(n)$ the projection onto the site $n \in \mathbb{Z}^{2}$ on $l^{2}\left(\mathbb{Z}^{2} ; \mathbb{C}^{2}\right)$ :

$$
(\hat{P}(n) \hat{f})(m)=\left(\begin{array}{c}
\delta_{n m} \hat{f}_{1}(m) \\
\delta_{n m} \hat{f}_{2}(m)
\end{array}\right),
$$

where $\delta_{n m}$ is Kronecker's delta. Throughout the paper, we shall assume that

(A). $\hat{q}$ is compactly supported, that is, $\hat{q}(n)=0$ except for a finite number of $n \in \mathbb{Z}^{2}$.

Note that some parts of our arguments are extended to more general decaying potentials.

We put

$$
q=\mathcal{F} \hat{q} \mathcal{F}^{*}, \tilde{q}=\mathcal{U} q \mathcal{U}^{*}
$$

Then $q$ and $\tilde{q}$ are written as

$$
\begin{gathered}
(q f)(\xi)=\frac{1}{2 \pi}\left(\begin{array}{l}
\int_{\mathbb{T}^{2}} q_{1}(\xi-\zeta) f_{1}(\zeta) d \zeta \\
\int_{\mathbb{T}^{2}} q_{2}(\xi-\zeta) f_{2}(\zeta) d \zeta
\end{array}\right), \\
(\tilde{q} f)(\xi)=\left(\begin{array}{c}
(\tilde{q} f)_{1}(\xi) \\
(\tilde{q} f)_{2}(\xi)
\end{array}\right),
\end{gathered}
$$

for $f=\left(f_{1}, f_{2}\right) \in L^{2}\left(\mathbb{T}^{2} ; \mathbb{C}^{2}\right)$, where

$$
q_{j}(\xi)=\frac{1}{2 \pi} \sum_{n \in \mathbb{Z}^{2}} \hat{q}_{j}(n) e^{i n \xi}, j \in\{1,2\},
$$

and

$$
\begin{aligned}
(\tilde{q} f)_{1}(\xi)= & \frac{1}{2(2 \pi)} \int_{\mathbb{T}^{2}}\left(q_{1}(\xi-\zeta)+\frac{\alpha(\xi)}{p(\xi)} q_{2}(\xi-\zeta) \frac{\bar{\alpha}(\zeta)}{p(\zeta)}\right) f_{1}(\zeta) d \zeta \\
& +\frac{1}{2(2 \pi)} \int_{\mathbb{T}^{2}}\left(q_{1}(\xi-\zeta)-\frac{\alpha(\xi)}{p(\xi)} q_{2}(\xi-\zeta) \frac{\bar{\alpha}(\zeta)}{p(\zeta)}\right) f_{2}(\zeta) d \zeta
\end{aligned}
$$




$$
\begin{aligned}
(\tilde{q} f)_{2}(\xi)= & \frac{1}{2(2 \pi)} \int_{\mathbb{T}^{2}}\left(q_{1}(\xi-\zeta)-\frac{\alpha(\xi)}{p(\xi)} q_{2}(\xi-\zeta) \frac{\bar{\alpha}(\zeta)}{p(\zeta)}\right) f_{1}(\zeta) d \zeta \\
& +\frac{1}{2(2 \pi)} \int_{\mathbb{T}^{2}}\left(q_{1}(\xi-\zeta)+\frac{\alpha(\xi)}{p(\xi)} q_{2}(\xi-\zeta) \frac{\bar{\alpha}(\zeta)}{p(\zeta)}\right) f_{2}(\zeta) d \zeta
\end{aligned}
$$

The discrete Schrödinger operator is denoted by

$$
\hat{H}=\hat{H}_{0}+\hat{q} .
$$

We also put

$$
\begin{aligned}
H & =\mathcal{F} \hat{H} \mathcal{F}^{*}=H_{0}+q, \\
\tilde{H} & =\mathcal{U} H \mathcal{U}^{*}=\tilde{H}_{0}+\tilde{q} .
\end{aligned}
$$

We denote by $\sigma_{e s s}(T)$ the essential spectrum of a self-adjoint operator $T$. Then, by noticing that the potential is a compact perturbation, we have

Proposition 2.3. $\sigma_{\text {ess }}(\tilde{H})=[-3,3]$.

\subsection{Sobolev spaces and Mourre estimates}

Let $L_{0}$ be the self-adjoint extension of the operator

$$
\left(\begin{array}{cc}
-\Delta & 0 \\
0 & -\Delta
\end{array}\right)
$$

on $C^{\infty}\left(\mathbb{T}^{2} ; \mathbb{C}^{2}\right)=\left\{\mathbb{C}^{2}\right.$-valued smooth function on $\left.\mathbb{T}^{2}\right\}$, where $\Delta=\left(\frac{\partial^{2}}{\partial \xi_{1}{ }^{2}}+\frac{\partial^{2}}{\partial \xi_{2}{ }^{2}}\right)$. We denote by $\mathcal{H}^{s}, s \in \mathbb{R}$, the domain of the operator $L_{0}^{s / 2}$ :

$$
\mathcal{H}^{s}=\left\{f \in \mathcal{D}^{\prime}\left(\mathbb{T}^{2} ; \mathbb{C}^{2}\right) ;\|f\|_{s}=\left\|\left(1+L_{0}\right)^{s / 2} f\right\|<\infty\right\},
$$

where $\mathcal{D}^{\prime}\left(\mathbb{T}^{2} ; \mathbb{C}^{2}\right)=\left\{\mathbb{C}^{2}\right.$-valued distribution on $\left.\mathbb{T}^{2}\right\}$. Also, we denote

$$
\hat{\mathcal{H}}^{s}=\mathcal{F}^{*} \mathcal{H}^{s} .
$$

We put $\mathcal{H}=\mathcal{H}^{0}=L^{2}\left(\mathbb{T}^{2} ; \mathbb{C}^{2}\right)$ and $\hat{\mathcal{H}}=\hat{\mathcal{H}}^{0}=l^{2}\left(\mathbb{Z}^{2} ; \mathbb{C}^{2}\right)$. The next proposition is obvious by Parseval's equation.

Proposition 2.4. $f \in \mathcal{H}^{s} \Longleftrightarrow \sum_{n \in \mathbb{Z}^{2}}\left(1+|n|^{2}\right)^{s}\|\hat{f}(n)\|_{\mathbb{C}^{2}}^{2}=\sum_{n \in \mathbb{Z}^{2}}(1+$ $\left.|n|^{2}\right)^{s}\left(\left|\hat{f}_{1}(n)\right|^{2}+\left|\hat{f}_{2}(n)\right|^{2}\right)<\infty$, where $f=\mathcal{F} \hat{f}$.

We derive Mourre estimates. At first, we perform formal calculations, and then modify the conjugate operator by introducing cut-off functions to prove its self-adjointness. Let $A$ be a first-order differential operator defined as

$$
\begin{aligned}
A & =i\left[\tilde{H}_{0}, L_{0}\right] \\
& =i\left(\begin{array}{cc}
\nabla p \cdot \nabla+\nabla \cdot \nabla p & 0 \\
0 & -(\nabla p \cdot \nabla+\nabla \cdot \nabla p)
\end{array}\right) .
\end{aligned}
$$


By a straightforward calculation, the commutator of $\tilde{H}_{0}$ and $A$ is

$$
i\left[\tilde{H}_{0}, A\right]=2|\nabla p(\xi)|^{2} I_{2},
$$

where $I_{2}$ is the $2 \times 2$ identity matrix.

A simple calculation shows that

$$
\nabla p(\xi)=\left(\frac{-\sin \xi_{1}-\sin \left(\xi_{1}-\xi_{2}\right)}{p(\xi)}, \frac{-\sin \xi_{2}+\sin \left(\xi_{1}-\xi_{2}\right)}{p(\xi)}\right) .
$$

If $\sin \xi_{1}+\sin \left(\xi_{1}-\xi_{2}\right)=0$, then

$$
\xi_{1}-\xi_{2}=\xi_{1}+\pi \text { or }-\xi_{1} \quad(\bmod 2 \pi) .
$$

Also, if $\sin \xi_{2}-\sin \left(\xi_{1}-\xi_{2}\right)=0$, then

$$
\xi_{1}-\xi_{2}=\xi_{2} \text { or }-\xi_{2}+\pi \quad(\bmod 2 \pi) .
$$

Note that we can not define $\nabla p(\xi)$ at $\xi_{0,1}$ and $\xi_{0,2}$. Therefore, we have

$$
\left\{\xi \in \mathbb{T}^{2} ; \nabla p(\xi)=0\right\}=\{(0,0),(0,-\pi),(-\pi, 0),(-\pi,-\pi)\} .
$$

From (6), $p(\xi)=1$ if and only if $\cos \left(\xi_{1}-\xi_{2}\right) / 2=0$ or $\cos \left(\xi_{1}-\xi_{2}\right) / 2=$ $-\cos \left(\xi_{1}+\xi_{2}\right) / 2$. If $\cos \left(\xi_{1}-\xi_{2}\right) / 2=0$, then

$$
\frac{\xi_{1}-\xi_{2}}{2}=\frac{\pi}{2} \quad(\bmod \pi)
$$

Also, if $\cos \left(\xi_{1}-\xi_{2}\right) / 2=-\cos \left(\xi_{1}+\xi_{2}\right) / 2$, then

$$
\frac{\xi_{1}-\xi_{2}}{2}= \pm \frac{\xi_{1}+\xi_{2}}{2}+\pi \quad(\bmod 2 \pi) .
$$

Therefore, we have

$$
\left\{\xi \in \mathbb{T}^{2} ; p(\xi)=1\right\}=\left\{\left(\xi_{1}, \xi_{2}\right) \in[-\pi, \pi)^{2} ; \xi_{1}=-\pi \text { or } \xi_{2}=-\pi \text { or } \xi_{2}=\xi_{1} \pm \pi\right\},
$$

which includes the set $\left\{\xi \in \mathbb{T}^{2} ; \nabla p(\xi)=0\right\}$ except the origin.

Let us define

$$
\mathcal{M}_{E}=\left\{\xi \in \mathbb{T}^{2} ; p(\xi)=E\right\} .
$$

Then we have

Proposition 2.5. For $E \in(0,3) \backslash\{1\}, \mathcal{M}_{E}$ is a real-analytic compact manifold.

Proof. We have $\nabla p(\xi) \neq 0$ on $\mathcal{M}_{E}$ for $E \in(0,3) \backslash\{1\}$. Noticing Proposition 2.1. we have the claim.

Put $C_{0}(E)=2 \inf _{\xi \in \mathcal{M}_{E}}|\nabla p(\xi)|^{2}$. Note that $C_{0}(E)>0$ for $E \in(0,3) \backslash\{1\}$. Then, for small $\varepsilon>0$, there is $\delta>0$ such that

$$
|\nabla p(\xi)|^{2} \geq C_{0}(E)-\varepsilon>0
$$


on $p^{-1}([E-\delta, E+\delta])$, which shall imply that

$$
i\left[\tilde{H}_{0}, A\right] \geq C_{0}(E)-\varepsilon .
$$

We have already shown that $p\left(\xi_{0, j}\right)=0, j \in\{1,2\}$, which implies that $\nabla p(\xi)$ has singularities at $\xi_{0,1}$ and $\xi_{0,2}$. To avoid them, we introduce a smooth cut-off function $\chi(\xi)$ : for small $\mu>0$,

$$
\chi(\xi)=\chi_{\mu}(\xi)= \begin{cases}0, & \text { if } \xi \in B_{\mu}\left(\xi_{0,1}\right) \cup B_{\mu}\left(\xi_{0,2}\right), \\ 1, & \text { if } \xi \notin B_{2 \mu}\left(\xi_{0,1}\right) \cup B_{2 \mu}\left(\xi_{0,2}\right),\end{cases}
$$

where $B_{\mu}\left(\xi_{0}\right)=\left\{\xi \in \mathbb{T}^{2} ;\left|\xi-\xi_{0}\right|<\mu\right\}$. Let us define

$$
A_{\chi}=\chi A \chi .
$$

Then, by Nelson's commutator theorem (Theorem X.37, Reed-Simon 23, where we take $\left.N=L_{0}+1\right), A_{\chi}$ is essentially self-adjoint on $C^{\infty}\left(\mathbb{T}^{2} ; \mathbb{C}^{2}\right)$.

Let us choose sufficiently small $\mu>0$ depending on $E$ and $\delta>0$. Then we have the Mourre estimate for $\tilde{H}_{0}$ :

$$
f\left(\tilde{H}_{0}\right) i\left[\tilde{H}_{0}, A_{\chi}\right] f\left(\tilde{H}_{0}\right) \geq\left(C_{0}(E)-\varepsilon\right) f\left(\tilde{H}_{0}\right)^{2}
$$

for any real-valued $f \in C_{0}^{\infty}((E-\delta, E+\delta))$. Figure 3 helps us to understand
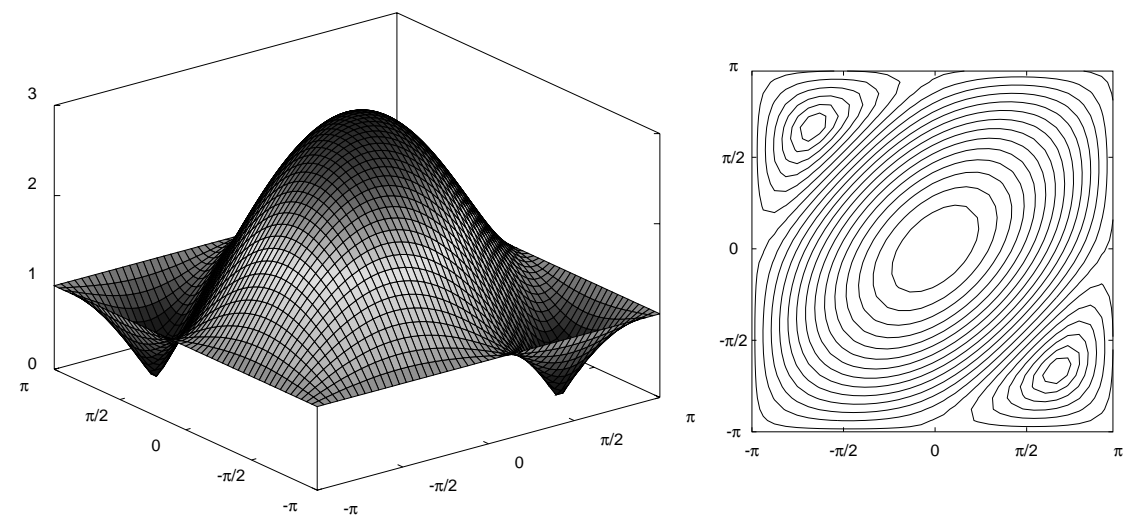

Figure 3: the surface graph and the contour lines of $p(\xi)$ on $[-\pi, \pi)^{2}$

what we have done so far in this section.

We can write $\left(i\left[\tilde{q}, A_{\chi}\right] f\right)(\xi)$ as a sum of the following terms: for some $a(\xi)$, $b(\xi), c(\xi), d(\xi) \in L^{\infty}\left(\mathbb{T}^{2}\right)$, and $j, k, l \in\{1,2\}$,

$$
a(\xi) \int_{\mathbb{T}^{2}} q_{j}(\xi-\zeta) b(\zeta) f_{k}(\zeta) d \zeta,
$$




$$
c(\xi) \int_{\mathbb{T}^{2}} \frac{\partial q_{j}}{\partial \xi_{l}}(\xi-\zeta) d(\zeta) f_{k}(\zeta) d \zeta
$$

Our assumption (A) makes those operators compact. As a result, we have the Mourre estimate for $\tilde{H}$, that is, there is a compact operator $K=K_{I}$ depending on the interval $I=(E-\delta / 2, E+\delta / 2)$ such that

$$
E_{\tilde{H}}(I)\left[\tilde{H}, i A_{\chi}\right] E_{\tilde{H}}(I) \geq\left(C_{0}(E)-\varepsilon\right) E_{\tilde{H}}(I)+K,
$$

where $E_{\tilde{H}}(\cdot)$ is the spectral projection for $\tilde{H}$.

Let $\tilde{R}(z)=(\tilde{H}-z)^{-1}$. We denote by $\mathbf{B}(X, Y)$, or simply $\mathbf{B}(X)$ when $X=Y$, the set of all bounded operators from $X$ to $Y$, where $X$ and $Y$ are Banach spaces. We also denote $D\left(\left(1+\left|A_{\chi}\right|\right)^{s}\right)$ equipped with graph norm by $\mathcal{H}_{A_{\chi}}$. Then, with the aid of (12) and (13), by the well-known Mourre theory (Mourre [18), we have the following theorem.

Theorem 2.1. Let $I_{0}=(-3,3) \backslash\{ \pm 1,0\}$. Then

1. The eigenvalues of $\tilde{H}$ are of finite multiplicities with possible accumulation points $0, \pm 1, \pm 3$.

2. There is no singularly continuous spectrum:

$$
\mathcal{H}=\mathcal{H}_{p p}(\tilde{H}) \oplus \mathcal{H}_{a c}(\tilde{H}) .
$$

3. Let $s>1 / 2$ and $\lambda \in I_{0}$. Then there is a norm limit $\tilde{R}(\lambda \pm i 0):=$ $\lim _{\varepsilon \searrow 0} \tilde{R}(\lambda \pm i \varepsilon)$ in $\mathbf{B}\left(\mathcal{H}_{A_{\chi}}^{s}, \mathcal{H}_{A_{\chi}}^{-s}\right)$, and $I_{0} \ni \lambda \rightarrow \tilde{R}(\lambda \pm i 0) \in \mathbf{B}\left(\mathcal{H}_{A_{\chi}}^{s}, \mathcal{H}_{A_{\chi}}^{-s}\right)$ is norm continuous. Furthermore, we have

$$
\sup _{\lambda \in J}\|\tilde{R}(\lambda \pm i 0)\|_{\mathbf{B}\left(\mathcal{H}_{A_{\chi}}^{s}, \mathcal{H}_{A_{\chi}}^{-s}\right)}<\infty
$$

for any compact interval $J$ in $I_{0} \backslash \sigma_{p p}(\tilde{H})$.

Corollary 2.1. If $s>1 / 2$,

$$
\sup _{\lambda \in J}\|\tilde{R}(\lambda \pm i 0)\|_{\mathbf{B}\left(\mathcal{H}^{s}, \mathcal{H}^{-s}\right)}<\infty .
$$

Proof of the Corollary. We only have to note that the inclusions $\mathcal{H}^{s} \subset \mathcal{H}_{A_{\chi}}^{s}$ and $\left(\mathcal{H}_{A_{\chi}}^{s}\right)^{*} \simeq \mathcal{H}_{A_{\chi}}^{-s} \subset \mathcal{H}^{-s} \simeq\left(\mathcal{H}^{s}\right)^{*}$ are continuous for $s>0$.

Conjugating by $\mathcal{U}$, we have the same statements for $H$; moreover, by $\mathcal{F}$, we also have those for $\hat{H}$, where we use $\hat{\mathcal{H}}^{s}$ instead of $\mathcal{H}^{s}$.

\section{Eigenoperators and scattering matrix}

\section{$3.1 \quad$ Trace operators}

Let $\tilde{R}_{0}(z)=\left(\tilde{H}_{0}-z\right)^{-1}$. Then we have

$$
\left(\tilde{R}_{0}(z) f, g\right)_{L^{2}\left(\mathbb{Z}^{2} ; \mathbb{C}^{2}\right)}=\int_{\mathbb{T}^{2}} \frac{f_{1}(\xi) \overline{g_{1}(\xi)}}{p(\xi)-z} d \xi+\int_{\mathbb{T}^{2}} \frac{f_{2}(\xi) \overline{g_{2}(\xi)}}{-p(\xi)-z} d \xi
$$


for $f=\left(f_{1}, f_{2}\right), g=\left(g_{1}, g_{2}\right) \in C^{\infty}\left(\mathbb{T}^{2} ; \mathbb{C}^{2}\right)$.

It has been already shown that $\mathcal{M}_{\lambda}$ is a real-analytic manifold for $\lambda \in$ $(0,3) \backslash\{1\}$. Thus we can introduce local coordinates $\omega$ on $\mathcal{M}_{\lambda}$, which induce the measure

$$
d \xi_{1} d \xi_{2}=d \mathcal{M}_{\lambda}(\omega) d \lambda=J(\lambda, \omega) d \omega d \lambda,
$$

where $J(\lambda, \omega)$ is real-analytic with respect to $\lambda$ and $\omega$.

We note that the set $\left\{\xi \in \mathbb{T}^{2} ; p(\xi) \in\{0,1,3\}\right\}$, which includes all the extreme points and the critical points of $p(\xi)$, has null Lebesgue measure. It enables us to write

$$
\begin{aligned}
\left(\tilde{R}_{0}(z) f, g\right)_{L^{2}\left(\mathbb{Z}^{2} ; \mathbb{C}^{2}\right)} & =\int_{0}^{3} \frac{h_{1}(\rho)}{\rho-z} d \rho+\int_{-3}^{0} \frac{h_{2}(-\rho)}{\rho-z} d \rho \\
& =\int_{-3}^{3} \frac{h(\rho)}{\rho-z} d \rho,
\end{aligned}
$$

where

$$
\begin{gathered}
h(\rho)= \begin{cases}h_{2}(-\rho), & \text { if } \rho \in(-3,0) \backslash\{-1\}, \\
h_{1}(\rho), & \text { if } \rho \in(0,3) \backslash\{1\},\end{cases} \\
h_{j}(\rho)=\int_{\mathcal{M}_{|\rho|}} f_{j}(\xi(\rho, \omega)) \overline{g_{j}(\xi(\rho, \omega))} d \mathcal{M}_{|\rho|}(\omega), j \in\{1,2\} .
\end{gathered}
$$

Then we have

$\left(\tilde{R}_{0}(\lambda \pm i 0) f, g\right)_{L^{2}\left(\mathbb{T}^{2} ; \mathbb{Z}^{2}\right)}= \pm i \pi h(\lambda)+$ p.v. $\int_{\lambda-\delta}^{\lambda-\delta} \frac{h(\rho)-h(\lambda)}{\rho-\lambda} d \rho+\int_{|\lambda-\rho|>\delta} \frac{h(\rho)}{\rho-\lambda} d \rho$

for $\lambda \in(-3,3) \backslash\{ \pm 1,0\}$, which leads us to

$$
\frac{1}{2 \pi i}\left(\left(\tilde{R}_{0}(\lambda+i 0)-\tilde{R}_{0}(\lambda-i 0)\right) f, g\right)_{L^{2}\left(\mathbb{T}^{2} ; \mathbb{C}^{2}\right)}=h(\lambda) .
$$

Let us define the trace operator $\tilde{\mathcal{F}}_{0}(\lambda)$ as

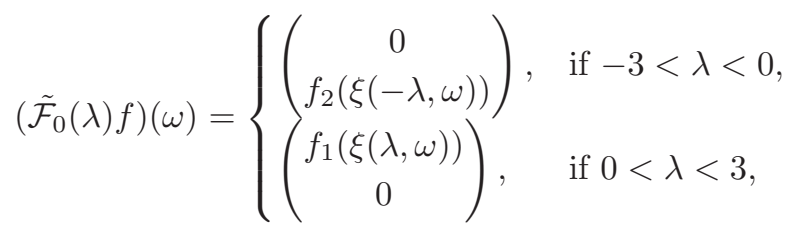

for $f \in C^{\infty}\left(\mathbb{T}^{2} ; \mathbb{C}^{2}\right)$. From (16), we have

Lemma 3.1. For $\lambda \in(-3,3) \backslash\{ \pm 1,0\}$,

$$
\frac{1}{2 \pi i}\left(\left(\tilde{R}_{0}(\lambda+i 0)-\tilde{R}_{0}(\lambda-i 0)\right) f, g\right)_{L^{2}\left(\mathbb{T}^{2} ; \mathbb{C}^{2}\right)}=\left(\tilde{\mathcal{F}}_{0}(\lambda) f, \tilde{\mathcal{F}}_{0}(\lambda) g\right)_{L^{2}\left(\mathcal{M}_{|\lambda|} ; \mathbb{C}^{2}\right)} .
$$

By Corollary 2.1 and this lemma, we have 
Corollary 3.1. For $s>1 / 2, \tilde{\mathcal{F}}_{0}(\lambda) \in \mathbf{B}\left(\mathcal{H}^{s}, L^{2}\left(\mathcal{M}_{|\lambda|} ; \mathbb{C}^{2}\right)\right)$.

Moreover, by Stone's formula, we have

Lemma 3.2. For $f, g \in \mathcal{H}^{s}, s>1 / 2$,

$$
(f, g)_{L^{2}\left(\mathbb{T}^{2} ; \mathbb{C}^{2}\right)}=\int_{-3}^{3}\left(\tilde{\mathcal{F}}_{0}(\lambda) f, \tilde{\mathcal{F}}_{0}(\lambda) g\right)_{L^{2}\left(\mathcal{M}_{|\lambda|} ; \mathbb{C}^{2}\right)} d \lambda .
$$

By the definition, we also have

$$
\tilde{\mathcal{F}}_{0}(\lambda)\left(\tilde{H}_{0}-\lambda\right)=0 .
$$

We pass from $\tilde{H}_{0}$ to $H_{0}$. For $s \in \mathbb{R}$ and small $\varepsilon>0$, let $\mathcal{H}_{\varepsilon}^{s}$ be the completion of $C_{\varepsilon}^{\infty}\left(\mathbb{T}^{2} ; \mathbb{C}^{2}\right)$ in $\mathcal{H}^{s}$, where

$$
C_{\varepsilon}^{\infty}\left(\mathbb{T}^{2} ; \mathbb{C}^{2}\right)=\left\{f \in C^{\infty}\left(\mathbb{T}^{2} ; \mathbb{C}^{2}\right) ; \operatorname{supp} f \subset \mathbb{T}^{2} \backslash\left(B_{\varepsilon}\left(\xi_{0,1}\right) \cup B_{\varepsilon}\left(\xi_{0,2}\right)\right)\right\} .
$$

Note that $\tilde{R}_{0}(z) C_{\varepsilon}^{\infty}\left(\mathbb{T}^{2} ; \mathbb{C}^{2}\right) \subset C_{\varepsilon}^{\infty}\left(\mathbb{T}^{2} ; \mathbb{C}^{2}\right)$. Since $\mathcal{H}_{\varepsilon}^{s}$ avoids the singular points of $\alpha(\xi) /|\alpha(\xi)|$, we have

Lemma 3.3. The operator $\mathcal{U}$ is defined as a homomorphism on $\mathcal{H}_{\varepsilon}^{s}$.

For sufficiently small $\varepsilon>0$, for example $0<\varepsilon<|\lambda| / 2$, we have $\tilde{R}_{0}(\lambda \pm$ $i 0) \in \mathbf{B}\left(L^{2}\left(B_{\varepsilon}\left(\xi_{0,1}\right) \cup B_{\varepsilon}\left(\xi_{0,2}\right) ; \mathbb{C}^{2}\right)\right)$; moreover, $\tilde{R}_{0}(\lambda+i 0) f=\tilde{R}_{0}(\lambda-i 0) f$ for $f \in L^{2}\left(B_{\varepsilon}\left(\xi_{0,1}\right) \cup B_{\varepsilon}\left(\xi_{0,2}\right) ; \mathbb{C}^{2}\right)$. Therefore, we can extend $\tilde{\mathcal{F}}_{0}(\lambda)$ uniquely as a null operator on $L^{2}\left(B_{\varepsilon}\left(\xi_{0,1}\right) \cup B_{\varepsilon}\left(\xi_{0,2}\right) ; \mathbb{C}^{2}\right)$.

Let $\mathcal{U}_{\lambda}^{\dagger}$ be a multiplication operator on $L^{2}\left(\mathcal{M}_{|\lambda|} ; \mathbb{C}^{2}\right)$ by the unitary matrix

$$
U^{\dagger}(\lambda, \omega)=\frac{1}{\sqrt{2}}\left(\begin{array}{cc}
1 & 1 \\
|\lambda|^{-1} \bar{\alpha}(\xi(|\lambda|, \omega)) & -|\lambda|^{-1} \bar{\alpha}(\xi(|\lambda|, \omega))
\end{array}\right),
$$

which means that $\mathcal{U}_{\lambda}^{\dagger}$ is a unitary operator. Then, from Lemma 3.3 and its subsequent descriptions, we can define the operator

$$
\mathcal{F}_{0}(\lambda)=\mathcal{U}_{\lambda}^{\dagger} \tilde{\mathcal{F}}_{0}(\lambda) \mathcal{U}
$$

which is written as

$$
\begin{aligned}
& \left(\mathcal{F}_{0}(\lambda) f\right)_{1}(\omega)=\frac{1}{2} f_{1}(\xi(|\lambda|, \omega))+\frac{1}{2} \lambda^{-1} \alpha(\xi(|\lambda|, \omega)) f_{2}(\xi(|\lambda|, \omega)), \\
& \left(\mathcal{F}_{0}(\lambda) f\right)_{2}(\omega)=\frac{1}{2} \lambda^{-1} \bar{\alpha}(\xi(|\lambda|, \omega)) f_{1}(\xi(|\lambda|, \omega))+\frac{1}{2} f_{2}(\xi(|\lambda|, \omega)),
\end{aligned}
$$

for $f=\left(f_{1}, f_{2}\right) \in \mathcal{H}^{s}$.

Lemma 3.4. For $s>1 / 2, \mathcal{F}_{0}(\lambda) \in \mathbf{B}\left(\mathcal{H}^{s}, L^{2}\left(\mathcal{M}_{|\lambda|} ; \mathbb{C}^{2}\right)\right)$.

Proof. Let $\chi=\chi_{\varepsilon} \in C^{\infty}\left(\mathbb{T}^{2}\right)$ be defined as (11). Then $1-\chi$ as a multiplication operator is continuous from $\mathcal{H}^{s}$ to $L^{2}\left(B_{\varepsilon}\left(\xi_{0,1}\right) \cup B_{\varepsilon}\left(\xi_{0,2}\right) ; \mathbb{C}^{2}\right)$; so is $\chi$ from $\mathcal{H}^{s}$ to $\mathcal{H}_{\varepsilon / 4}^{s}$, which proves the lemma. 
Let $R_{0}(z)=\left(H_{0}-z\right)^{-1}$. Passing through $\mathcal{U}_{\lambda}^{\dagger}$ and $\mathcal{U}$ in Lemmas 3.1 and 3.2 we have the following two lemmas.

Lemma 3.5. For $f, g \in \mathcal{H}^{s}, s>1 / 2$,

$$
\frac{1}{2 \pi i}\left(\left(R_{0}(\lambda+i 0)-R_{0}(\lambda-i 0)\right) f, g\right)_{L^{2}\left(\mathbb{T}^{2} ; \mathbb{C}^{2}\right)}=\left(\mathcal{F}_{0}(\lambda) f, \mathcal{F}_{0}(\lambda) g\right)_{L^{2}\left(\mathcal{M}_{|\lambda|} ; \mathbb{C}^{2}\right)},
$$

Lemma 3.6. For $f, g \in \mathcal{H}^{s}, s>1 / 2$,

$$
(f, g)_{L^{2}\left(\mathbb{T}^{2} ; \mathbb{C}^{2}\right)}=\int_{-3}^{3}\left(\mathcal{F}_{0}(\lambda) f, \mathcal{F}_{0}(\lambda) g\right)_{L^{2}\left(\mathcal{M}_{|\lambda|} ; \mathbb{C}^{2}\right)} d \lambda .
$$

From (17), we also have

$$
\mathcal{F}_{0}(\lambda)\left(H_{0}-\lambda\right)=0 .
$$

For $\lambda \in(-3,3) \backslash\left(\{ \pm 1,0\} \cup \sigma_{p p}(H)\right)$, let us define the operator

$$
\mathcal{F}^{( \pm)}(\lambda)=\mathcal{F}_{0}(\lambda)(I-q R(\lambda \pm i 0)) .
$$

Then we have

Lemma 3.7. For $s>1 / 2$ and $\lambda \in(-3,3) \backslash\left(\{ \pm 1,0\} \cup \sigma_{p p}(H)\right)$,

$$
\mathcal{F}^{( \pm)}(\lambda) \in \mathbf{B}\left(\mathcal{H}^{s}, L^{2}\left(\mathcal{M}_{|\lambda|} ; \mathbb{C}^{2}\right)\right) .
$$

Proof. Noticing that $q \in \mathbf{B}\left(\mathcal{H}^{-s}, \mathcal{H}^{s}\right)$, this is an immediate consequence of Corollary 2.1 and Lemma 3.4 .

By the definition of $\mathcal{F}^{( \pm)}(\lambda)$ and (18), we have

$$
\mathcal{F}^{( \pm)}(\lambda)(H-\lambda)=0 .
$$

The next lemma is proved by calculating $2 \pi i \mathcal{F}^{( \pm)}(\lambda)^{*} \mathcal{F}^{( \pm)}(\lambda)$ in the same way as in Lemma 3.3 of Isozaki and Korotyaev [9].

Lemma 3.8. For $f, g \in \mathcal{H}^{s}, s>1 / 2$,

$$
\frac{1}{2 \pi i}((R(\lambda+i 0)-R(\lambda-i 0)) f, g)_{L^{2}\left(\mathbb{T}^{2} ; \mathbb{C}^{2}\right)}=\left(\mathcal{F}^{( \pm)}(\lambda) f, \mathcal{F}^{( \pm)}(\lambda) g\right)_{L^{2}\left(\mathcal{M}_{|\lambda|} ; \mathbb{C}^{2}\right)} .
$$

\subsection{Eigenoperators and spectral representations}

The adjoint operator $\mathcal{F}_{0}(\lambda)^{*}$ is defined by

$$
\left(\mathcal{F}_{0}(\lambda) f, \phi\right)_{L^{2}\left(\mathcal{M}_{|\lambda|} ; \mathbb{C}^{2}\right)}=\left(f, \mathcal{F}_{0}(\lambda)^{*} \phi\right)_{L^{2}\left(\mathbb{T}^{2} ; \mathbb{C}^{2}\right)}
$$

for $f \in \mathcal{H}^{s}, s>1 / 2$, and $\phi \in L^{2}\left(\mathcal{M}_{|\lambda|} ; \mathbb{C}^{2}\right)$. By the definition, we have $\mathcal{F}_{0}(\lambda)^{*} \in$ $\mathbf{B}\left(L^{2}\left(\mathcal{M}_{|\lambda|} ; \mathbb{C}^{2}\right), \mathcal{H}^{-s}\right)$, which is written as

$$
\begin{aligned}
& \left(\mathcal{F}_{0}(\lambda)^{*} \phi\right)_{1}(\xi(|\rho|, \omega)) \\
& =\frac{1}{2} \delta(p(\xi(|\rho|, \omega))-|\lambda|) \phi_{1}(\omega)+\frac{1}{2} \frac{\alpha(\xi(|\rho|, \omega))}{\lambda} \delta(p(\xi(|\rho|, \omega))-|\lambda|) \phi_{2}(\omega),
\end{aligned}
$$




$$
\begin{aligned}
& \left(\mathcal{F}_{0}(\lambda)^{*} \phi\right)_{2}(\xi(|\rho|, \omega)) \\
& =\frac{1}{2} \frac{\bar{\alpha}(\xi(|\rho|, \omega))}{\lambda} \delta(p(\xi(|\rho|, \omega))-|\lambda|) \phi_{1}(\omega)+\frac{1}{2} \delta(p(\xi(|\rho|, \omega)-|\lambda|)) \phi_{2}(\omega),
\end{aligned}
$$

for $\phi=\left(\phi_{1}(\omega), \phi_{2}(\omega)\right) \in C^{\infty}\left(\mathcal{M}_{|\lambda|} ; \mathbb{C}^{2}\right)$, where $\delta(\cdot)$ is Dirac's delta.

By taking the adjoint of (18), $\mathcal{F}_{0}(\lambda)^{*}$ is the eigenoperator of $H_{0}$ :

$$
\left(H_{0}-\lambda\right) \mathcal{F}_{0}(\lambda)^{*}=0 .
$$

Let us define the operator $\mathcal{F}_{0}$ by

$$
\left(\mathcal{F}_{0} f\right)(\lambda, \omega)=\left(\mathcal{F}_{0}(\lambda) f\right)(\omega)
$$

for $f \in \mathcal{H}^{s}, s>1 / 2$. The proof of the next theorem as well as that for Theorem 3.2 can be done in the same way as in Isozaki and Korotyaev [9. We shall give the sketch in Appendix A for the reader's convenience.

Theorem 3.1. $\quad$ 1. $\mathcal{F}_{0}$ is uniquely extended to a unitary operator from $L^{2}\left(\mathbb{T}^{2} ; \mathbb{C}^{2}\right)$ to $L^{2}\left((-3,3) ; L^{2}\left(\mathcal{M}_{|\lambda|} ; \mathbb{C}^{2}\right) ; d \lambda\right)$.

2. $\mathcal{F}_{0}$ diagonalizes $H_{0}$ :

$$
\left(\mathcal{F}_{0} H_{0} f\right)(\lambda)=\lambda\left(\mathcal{F}_{0} f\right)(\lambda) .
$$

3. For any compact interval $I \subset(-3,3) \backslash\{ \pm 1,0\}$,

$$
\int_{I} \mathcal{F}_{0}(\lambda)^{*} g(\lambda) d \lambda \in L^{2}\left(\mathbb{T}^{2} ; \mathbb{C}^{2}\right)
$$

for $g \in L^{2}\left((-3,3) ; L^{2}\left(\mathcal{M}_{|\lambda|} ; \mathbb{C}^{2}\right) ; d \lambda\right)$. Moreover, the following inversion formula holds:

$$
f=s-\lim _{N \rightarrow \infty} \int_{I_{N}} \mathcal{F}_{0}(\lambda)^{*}\left(\mathcal{F}_{0} f\right)(\lambda) d \lambda
$$

for $f \in L^{2}\left(\mathbb{T}^{2} ; \mathbb{C}^{2}\right)$, where $I_{N}$ is a finite union of compact intervals in $(-3,3) \backslash\{ \pm 1,0\}$ such that $I_{N} \rightarrow(-3,3)$ as $N \rightarrow \infty$.

Let us also define the operators $\mathcal{F}^{( \pm)}$as

$$
\left(\mathcal{F}^{( \pm)} f\right)(\lambda, \omega)=\left(\mathcal{F}^{( \pm)}(\lambda) f\right)(\omega)
$$

for $f \in \mathcal{H}^{s}, s>1 / 2$.

Theorem 3.2. $\quad$ 1. $\mathcal{F}^{( \pm)}$are uniquely extended to partial isometries from $L^{2}\left(\mathbb{T}^{2} ; \mathbb{C}^{2}\right)$ to $L^{2}\left((-3,3) ; L^{2}\left(\mathcal{M}_{|\lambda|} ; \mathbb{C}\right) ; d \lambda\right)$ with the initial set $\mathcal{H}_{a c}(H)$ and the final set $L^{2}\left((-3,3) ; L^{2}\left(\mathcal{M}_{|\lambda|} ; \mathbb{C}^{2}\right) ; d \lambda\right)$. Moreover, $\mathcal{F}^{( \pm)}$diagonalize $H$ :

$$
\left(\mathcal{F}^{( \pm)} H f\right)(\lambda)=\lambda\left(\mathcal{F}^{( \pm)} f\right)(\lambda)
$$

for $f \in L^{2}\left(\mathbb{T}^{2} ; \mathbb{C}^{2}\right)$. 
2. The following inversion formula holds:

$$
f=s-\lim _{N \rightarrow \infty} \int_{I_{N}} \mathcal{F}^{( \pm)}(\lambda)^{*}\left(\mathcal{F}^{( \pm)} f\right)(\lambda) d \lambda
$$

for $f \in \mathcal{H}_{a c}(H)$, where $I_{N}$ is a finite union of compact intervals in $(-3,3) \backslash$ $\left(\{ \pm 1,0\} \cup \sigma_{p p}(H)\right)$ such that $I_{N} \rightarrow(-3,3)$ as $N \rightarrow \infty$.

3. $\mathcal{F}^{( \pm)} \in \mathbf{B}\left(L^{2}\left(\mathcal{M}_{|\lambda|} ; \mathbb{C}^{2}\right), \mathcal{H}^{-s}\right)$ are the eigenoperators for $H$ in the following sense:

$$
(H-\lambda) \mathcal{F}^{( \pm)}(\lambda)^{*} \phi=0
$$

for $\phi \in L^{2}\left(\mathcal{M}_{|\lambda|} ; \mathbb{C}^{2}\right)$.

Now, we turn to the spectral representation for $\hat{H}$ on the lattice. For $\omega \in$ $\mathcal{M}_{|\lambda|}$, let us define the distribution $\delta_{\omega}(\cdot) \in \mathcal{D}^{\prime}\left(\mathcal{M}_{|\lambda|}\right)$ by

$$
\left\langle\delta_{\omega}, \phi\right\rangle=\phi(\omega)
$$

for $\phi \in C^{\infty}\left(\mathcal{M}_{|\lambda|}\right)$. Note that $\mathcal{M}_{|\lambda|}$ is an analytic manifold for $\lambda \in(-3,3) \backslash$ $\{ \pm 1,0\}$. Then, from (20) and (21), we have the matrix-valued distribution kernel of $\mathcal{F}_{0}(\lambda)^{*}$, which is written as

$$
\begin{aligned}
\psi^{(0)}(\xi ; \lambda, \theta) & =\psi^{(0)}(\xi(|\rho|, \omega) ; \lambda, \theta) \\
& =\frac{1}{2}\left(\begin{array}{cc}
1 & \lambda^{-1} \alpha(\xi(|\rho|, \omega)) \\
\lambda^{-1} \bar{\alpha}(\xi(|\rho|, \omega)) & 1
\end{array}\right) \delta(p(\xi(|\rho|, \omega))-|\lambda|) \otimes \delta_{\omega}(\theta),
\end{aligned}
$$

where $\omega \in \mathcal{M}_{|\rho|}$ and $\theta \in \mathcal{M}_{|\lambda|}$.

In the lattice space, we consider the Fourier coefficients of $\psi^{(0)}(\xi ; \lambda, \theta)$ :

$$
\hat{\psi}(\lambda, \theta)=\left(\hat{\psi}^{(0)}(n ; \lambda, \theta)\right)_{n \in \mathbb{Z}^{2}},
$$

where

$$
\begin{aligned}
\hat{\psi}^{(0)}(n ; \lambda, \theta) & =\frac{1}{2 \pi} \int_{\mathbb{T}^{2}} \psi^{(0)}(\xi ; \lambda, \theta) e^{-i n \xi} d \xi \\
& =\frac{1}{2} \frac{1}{2 \pi} J(|\lambda|, \theta) e^{i n \xi(|\lambda|, \theta)}\left(\begin{array}{cc}
1 & \lambda^{-1} \alpha(\xi(|\lambda|, \theta)) \\
\lambda^{-1} \bar{\alpha}(\xi(|\lambda|, \theta)) & 1
\end{array}\right),
\end{aligned}
$$

and $J(|\lambda|, \theta)$ is the density introduced in (15). We put

$$
\hat{\mathcal{F}}_{0}=\mathcal{F}_{0} \mathcal{F} .
$$

Then we have

$$
\begin{aligned}
& \left(\mathcal{F}_{0}(\lambda) \mathcal{F} \hat{f}\right)_{1}(\theta) \\
= & \frac{1}{2} \frac{1}{2 \pi} \sum_{n \in \mathbb{Z}^{2}} e^{i n \xi(|\lambda|, \theta)} \hat{f}_{1}(n)+\frac{1}{2} \frac{1}{2 \pi} \frac{\alpha(\xi(|\lambda|, \theta))}{\lambda} \sum_{n \in \mathbb{Z}^{2}} e^{i n \xi(|\lambda|, \theta)} \hat{f}_{2}(n),
\end{aligned}
$$




$$
\begin{aligned}
& \left(\mathcal{F}_{0}(\lambda) \mathcal{F} \hat{f}\right)_{2}(\theta) \\
= & \frac{1}{2} \frac{1}{2 \pi} \frac{\bar{\alpha}(\xi(|\lambda|, \theta))}{\lambda} \sum_{n \in \mathbb{Z}^{2}} e^{i n \xi(|\lambda|, \theta)} \hat{f}_{1}(n)+\frac{1}{2} \frac{1}{2 \pi} \sum_{n \in \mathbb{Z}^{2}} e^{i n \xi(|\lambda|, \theta)} \hat{f}_{2}(n),
\end{aligned}
$$

for $\hat{f}=\left(\hat{f}_{1}, \hat{f}_{2}\right) \in \hat{\mathcal{H}}^{s}, s>1 / 2$. Also, we have

$$
\hat{\mathcal{F}}_{0}^{*} \phi(\lambda)=\left(\hat{\mathcal{F}}_{0}^{*} \phi(n ; \lambda)\right)_{n \in \mathbb{Z}^{2}}
$$

for $\phi=\left(\phi_{1}, \phi_{2}\right) \in L^{2}\left(\mathcal{M}_{|\lambda|} ; \mathbb{C}^{2}\right)$, where

$$
\begin{aligned}
& \hat{\mathcal{F}}_{0}^{*} \phi(n ; \lambda)=\left(\hat{\mathcal{F}}_{0}(\lambda)^{*} \phi\right)(n) \\
&= \int_{\mathcal{M}_{|\lambda|}} \hat{\psi}^{(0)}(n ; \lambda, \theta) \phi(\theta) d \mathcal{M}_{|\lambda|}(\theta) \\
&=\left(\begin{array}{l}
\left(\hat{\mathcal{F}}_{0}(\lambda)^{*} \phi\right)_{1}(n) \\
\left(\hat{\mathcal{F}}_{0}(\lambda)^{*} \phi\right)_{2}(n)
\end{array}\right), \\
&\left(\hat{\mathcal{F}}_{0}(\lambda)^{*} \phi\right)_{1}(n)=\frac{1}{2 \pi} \int_{\mathcal{M}_{|\lambda|}} \frac{1}{2} e^{-i n \xi(|\lambda|, \theta)} \phi_{1}(\theta) d \mathcal{M}_{|\lambda|}(\theta) \\
&+\frac{1}{2 \pi} \int_{\mathcal{M}_{|\lambda|}} \frac{1}{2} \frac{\alpha(\xi(|\lambda|, \theta))}{\lambda} e^{-i n \xi(|\lambda|, \theta)} \phi_{2}(\theta) d \mathcal{M}_{|\lambda|}(\theta), \\
&\left(\hat{\mathcal{F}}_{0}(\lambda)^{*} \phi\right)_{2}(n)= \\
&+\frac{1}{2 \pi} \int_{\mathcal{M}_{|\lambda|}} \frac{1}{2} \frac{\bar{\alpha}(\xi(|\lambda|, \theta))}{\lambda} e^{-i n \xi(|\lambda|, \theta)} \phi_{1}(\theta) d \mathcal{M}_{|\lambda|}(\theta) \\
&+\frac{1}{2 \pi} \int_{\mathcal{M}_{|\lambda|}} \frac{1}{2} e^{-i n \xi(|\lambda|, \theta)} \phi_{2}(\theta) d \mathcal{M}_{|\lambda|}(\theta) .
\end{aligned}
$$

A direct calculation shows that the resolvent $R_{0}(z)=\left(H_{0}-z\right)^{-1}$ is a multiplication operator by the matrix

$$
R_{0}(z, \xi)=\frac{1}{z^{2}-|\alpha(\xi)|}\left(\begin{array}{cc}
-z & -\alpha(\xi) \\
-\bar{\alpha}(\xi) & -z
\end{array}\right)
$$

Let $\hat{r}_{0}(z)=\left(\hat{r}_{0}(z, n)\right)_{n \in \mathbb{Z}^{2}}$ be the Fourier coefficients of $R_{0}(z, \xi)$, which is written as

$$
\begin{aligned}
\hat{r}_{0}(z, n) & =\left(\begin{array}{ll}
\hat{r}_{0,11}(z, n) & \hat{r}_{0,12}(z, n) \\
\hat{r}_{0,21}(z, n) & \hat{r}_{0,22}(z, n)
\end{array}\right) \\
& =\frac{1}{2 \pi} \int_{\mathbb{T}^{2}} R_{0}(z, \xi) e^{-i n \xi} d \xi
\end{aligned}
$$


Then the resolvent $\hat{R}_{0}(z)=\left(\hat{H}_{0}-z\right)^{-1}$ is a convolution operator by $\hat{r}_{0}(z)$ :

$$
\begin{aligned}
\left(\hat{R}_{0}(z) \hat{f}\right)(n)= & \left(\begin{array}{l}
\left(\hat{r}_{0,11}(z) * \hat{f}_{1}\right)(n)+\left(\hat{r}_{0,12}(z) * \hat{f}_{2}\right)(n) \\
\left(\hat{r}_{0,21}(z) * \hat{f}_{1}\right)(n)+\left(\hat{r}_{0,22}(z) * \hat{f}_{2}\right)(n)
\end{array}\right) \\
= & \left(\begin{array}{l}
\sum_{m \in \mathbb{Z}^{2}} \hat{r}_{0,11}(z, n-m) \hat{f}_{1}(m)+\sum_{m \in \mathbb{Z}^{2}} \hat{r}_{0,12}(z, n-m) \hat{f}_{2}(m) \\
\sum_{m \in \mathbb{Z}^{2}} \hat{r}_{0,21}(z, n-m) \hat{f}_{1}(m)+\sum_{m \in \mathbb{Z}^{2}} \hat{r}_{0,22}(z, n-m) \hat{f}_{2}(m)
\end{array}\right),
\end{aligned}
$$

for $\hat{f}=\left(\hat{f}_{1}, \hat{f}_{2}\right) \in l^{2}\left(\mathbb{Z}^{2} ; \mathbb{C}^{2}\right)$.

We put

$$
\hat{\mathcal{F}}^{( \pm)}=\mathcal{F}^{( \pm)} \mathcal{F}
$$

Passing to the Fourier transform $\mathcal{F}$ in Theorem 3.2 we have

Theorem 3.3. $\quad$ 1. $\hat{\mathcal{F}}^{( \pm)}$are uniquely extended to partial isometries from $l^{2}\left(\mathbb{Z}^{2} ; \mathbb{C}^{2}\right)$ to $L^{2}\left((-3,3) ; L^{2}\left(\mathcal{M}_{|\lambda|} ; \mathbb{C}^{2}\right) ; d \lambda\right)$ with the initial set $\mathcal{H}_{a c}(\hat{H})$ and the final set $L^{2}\left((-3,3) ; L^{2}\left(\mathcal{M}_{|\lambda|} ; \mathbb{C}^{2}\right) ; d \lambda\right)$. Moreover, $\hat{\mathcal{F}}^{( \pm)}$diagonalize $\hat{H}$ :

$$
\left(\hat{\mathcal{F}}^{( \pm)} \hat{H} \hat{f}\right)(\lambda)=\lambda\left(\hat{\mathcal{F}}^{( \pm)} \hat{f}\right)(\lambda)
$$

for $f \in l^{2}\left(\mathbb{Z}^{2} ; \mathbb{C}^{2}\right)$

2. The following inversion formula holds:

$$
\hat{f}=s-\lim _{N \rightarrow \infty} \int_{I_{N}} \hat{\mathcal{F}}^{( \pm)}(\lambda)^{*}\left(\hat{\mathcal{F}}^{( \pm)} \hat{f}\right)(\lambda) d \lambda
$$

for $f \in \mathcal{H}_{a c}(\hat{H})$, where $I_{N}$ is a finite union of compact intervals in $(-3,3) \backslash$ $\left(\{ \pm 1,0\} \cup \sigma_{p p}(H)\right)$ such that $I_{N} \rightarrow(-3,3)$ as $N \rightarrow \infty$.

3. $\hat{\mathcal{F}}^{( \pm)}$diagonalize $\hat{H}$ in the following sense:

$$
\left(\hat{\mathcal{F}}^{( \pm)} \hat{H} \hat{f}\right)(\lambda)=\lambda\left(\hat{\mathcal{F}}^{( \pm)} \hat{f}\right)(\lambda)
$$

for $\hat{f} \in \mathcal{H}_{a c}(\hat{H})$.

\subsection{Scattering matrix}

Since $\hat{q}(n)$ is compactly supported, we can use the trace class perturbation theory for $\hat{H}$ and $\hat{H}_{0}$, that is, the wave operators

$$
W_{ \pm}=\mathrm{s}-\lim _{t \pm \infty} e^{i t \hat{H}} e^{-i t \hat{H}_{0}}
$$

exist, and they are complete (Theorem XI.8, Reed-Simon [23]). The scattering operator is defined by

$$
S=W_{+}^{*} W_{-} .
$$


We put

$$
\hat{S}=\hat{\mathcal{F}}_{0} S \hat{\mathcal{F}}_{0}^{*} .
$$

Since $S$ commutes with $\hat{H}_{0}$ and $\hat{\mathcal{F}}_{0}$ diagonalizes $\hat{H}_{0}, \hat{S}$ is decomposable on $L^{2}\left((-3,3) ; L^{2}\left(\mathcal{M}_{|\lambda|} ; \mathbb{C}^{2}\right) ; d \lambda\right)=\int_{-3}^{3} \oplus L^{2}\left(\mathcal{M}_{|\lambda|} ; \mathbb{C}^{2}\right) d \lambda$ :

$$
\hat{S}=\int_{-3}^{3} \oplus \hat{S}(\lambda) d \lambda
$$

where $\hat{S}(\lambda)$ is the S-matrix, which is unitary on $L^{2}\left(\mathcal{M}_{|\lambda|} ; \mathbb{C}^{2}\right)$.

Let us define the scattering amplitude $A(\lambda)$ by

$$
\hat{S}(\lambda)=I-2 \pi i A(\lambda) .
$$

Then, by using abstract stationary scattering theory (Kato and Kuroda [12, Kuroda [15, 16]), we have

$$
A(\lambda)=\hat{\mathcal{F}}_{0}(\lambda) \hat{q} \hat{\mathcal{F}}_{0}(\lambda)^{*}-\hat{\mathcal{F}}_{0}(\lambda) \hat{q} \hat{R}(\lambda+i 0) \hat{q} \hat{\mathcal{F}}_{0}(\lambda)^{*} .
$$

Let $A\left(\lambda, \theta, \theta^{\prime}\right)$ be the integral kernel of $A(\lambda)$, which is written as

$$
A\left(\lambda, \theta, \theta^{\prime}\right)=\left(\begin{array}{ll}
A_{11}\left(\lambda, \theta, \theta^{\prime}\right) & A_{12}\left(\lambda, \theta, \theta^{\prime}\right) \\
A_{21}\left(\lambda, \theta, \theta^{\prime}\right) & A_{22}\left(\lambda, \theta, \theta^{\prime}\right)
\end{array}\right) .
$$

The potential $\hat{q}$ is a multiplication operator by the $2 \times 2$ matrices $(\hat{q}(n))_{n \in \mathbb{Z}^{2}}$ defined by (8), and the action of $\hat{R}(z)$ has already been given by (28) and (29). Then, by using (24), (25), (26) and (27), we have the following representations for $A_{j j}\left(\lambda, \theta, \theta^{\prime}\right), j \in\{1,2\}$ :

$$
\begin{aligned}
& A_{11}\left(\lambda, \theta, \theta^{\prime}\right) \\
= & \frac{1}{4} \frac{1}{(2 \pi)^{2}} J(|\lambda|, \theta) J\left(|\lambda|, \theta^{\prime}\right) \sum_{n \in \mathbb{Z}^{2}} e^{i n\left(\xi(|\lambda|, \theta)-\xi\left(|\lambda|, \theta^{\prime}\right)\right)} \\
& \cdot\left(\hat{q}_{1}(n)+\frac{\alpha(\xi(|\lambda|, \theta))}{\lambda} \frac{\bar{\alpha}\left(\xi\left(|\lambda|, \theta^{\prime}\right)\right)}{\lambda} \hat{q}_{2}(n)\right) \\
+ & \frac{1}{2} \frac{1}{2 \pi} J(|\lambda|, \theta)\left(\sum_{n \in \mathbb{Z}^{2}} e^{i n \xi(|\lambda|, \theta)} \hat{q}_{1}(n)\left(\hat{R}(\lambda+i 0) \hat{q} \hat{\psi}^{(0)}\left(\lambda, \theta^{\prime}\right)\right)_{11}(n)\right. \\
& \left.+\frac{\alpha(\xi(|\lambda|, \theta))}{\lambda} \sum_{n \in \mathbb{Z}^{2}} e^{i n \xi(|\lambda|, \theta)} \hat{q}_{2}(n)\left(\hat{R}(\lambda+i 0) \hat{q} \hat{\psi}^{(0)}\left(\lambda, \theta^{\prime}\right)\right)_{21}(n)\right),
\end{aligned}
$$




$$
\begin{aligned}
& A_{22}\left(\lambda, \theta, \theta^{\prime}\right) \\
= & \frac{1}{4} \frac{1}{(2 \pi)^{2}} J(|\lambda|, \theta) J\left(|\lambda|, \theta^{\prime}\right) \sum_{n \in \mathbb{Z}^{2}} e^{i n\left(\xi(|\lambda|, \theta)-\xi\left(|\lambda|, \theta^{\prime}\right)\right)} \\
& \cdot\left(\frac{\bar{\alpha}(\xi(|\lambda|, \theta))}{\lambda} \frac{\alpha\left(\xi\left(|\lambda|, \theta^{\prime}\right)\right)}{\lambda} \hat{q}_{1}(n)+\hat{q}_{2}(n)\right) \\
+ & \frac{1}{2} \frac{1}{2 \pi} J(|\lambda|, \theta)\left(\frac{\bar{\alpha}(\xi(|\lambda|, \theta))}{\lambda} \sum_{n \in \mathbb{Z}^{2}} e^{i n \xi(|\lambda|, \theta)} \hat{q}_{1}(n)\left(\hat{R}(\lambda+i 0) \hat{q} \hat{\psi}^{(0)}\left(\lambda, \theta^{\prime}\right)\right)_{12}(n)\right. \\
& \left.+\sum_{n \in \mathbb{Z}^{2}} e^{i n \xi(|\lambda|, \theta)} \hat{q}_{2}(n)\left(\hat{R}(\lambda+i 0) \hat{q} \hat{\psi}^{(0)}\left(\lambda, \theta^{\prime}\right)\right)_{22}(n)\right) .
\end{aligned}
$$

We omit the formulas for $A_{i j}\left(\lambda, \theta, \theta^{\prime}\right),(i, j)=(1,2)$ or $(2,1)$, since we do not use them later.

\section{Analytic continuation}

A simple calculation shows that if $\xi \in \mathcal{M}_{\lambda}$

$$
\cos ^{2} \frac{\xi_{1}}{2} \cos ^{2} \frac{\xi_{2}}{2}+\sin \frac{\xi_{1}}{2} \sin \frac{\xi_{2}}{2} \cos \frac{\xi_{1}}{2} \cos \frac{\xi_{2}}{2}=\frac{1}{8}\left(\lambda^{2}-1\right),
$$

which is rewritten as

$$
\left(\cos \frac{\xi_{1}}{2} \cos \frac{\xi_{2}}{2}+\frac{1}{2} \sin \frac{\xi_{1}}{2} \sin \frac{\xi_{2}}{2}\right)^{2}-\left(\frac{1}{2} \sin \frac{\xi_{1}}{2} \sin \frac{\xi_{2}}{2}\right)^{2}=\frac{1}{8}\left(\lambda^{2}-1\right) .
$$

We let $\lambda=\sqrt{8 k^{2}+1}$ so that $k$ varies over $(-1,0) \cup(0,1)$. Then we have

$$
\begin{gathered}
\cos \frac{\xi_{1}}{2} \cos \frac{\xi_{2}}{2}+\frac{1}{2} \sin \frac{\xi_{1}}{2} \sin \frac{\xi_{2}}{2}= \pm k \cosh \theta \\
\frac{1}{2} \sin \frac{\xi_{1}}{2} \sin \frac{\xi_{2}}{2}= \pm k \sinh \theta
\end{gathered}
$$

where $\theta \in \mathbb{R}$. We parametrize $\xi \in \mathcal{M}_{\lambda}$ by using (31) and (32), for which we need to solve (31) and (32) with respect to $\xi_{1}$ and $\xi_{2}$.

From (31) and (32), we have

$$
\begin{gathered}
\cos ^{2} \frac{\xi_{1}}{2} \cos ^{2} \frac{\xi_{2}}{2}=k^{2} e^{-2 \theta}, \\
\sin ^{2} \frac{\xi_{1}}{2} \sin ^{2} \frac{\xi_{2}}{2}=4 k^{2} \sinh ^{2} \theta .
\end{gathered}
$$

By solving (33) and (34) with respect to $\sin ^{2} \frac{\xi_{j}}{2}$ and $\cos ^{2} \frac{\xi_{j}}{2}, j \in\{1,2\}$, we put

$$
\sin ^{2} \frac{\xi_{1}}{2}=\frac{1}{2}\left\{1-\left(2-e^{2 \theta}\right) k^{2}+\sqrt{\left(2-e^{2 \theta}\right)^{2} k^{4}-2\left(2 e^{-2 \theta}-\left(2-e^{2 \theta}\right)\right) k^{2}+1}\right\},
$$




$$
\begin{aligned}
& \sin ^{2} \frac{\xi_{2}}{2}=\frac{1}{2}\left\{1-\left(2-e^{2 \theta}\right) k^{2}-\sqrt{\left(2-e^{2 \theta}\right)^{2} k^{4}-2\left(2 e^{-2 \theta}-\left(2-e^{2 \theta}\right)\right) k^{2}+1}\right\}, \\
& \cos ^{2} \frac{\xi_{1}}{2}=\frac{1}{2}\left\{1+\left(2-e^{2 \theta}\right) k^{2}-\sqrt{\left(2-e^{2 \theta}\right)^{2} k^{4}-2\left(2 e^{-2 \theta}-\left(2-e^{2 \theta}\right)\right) k^{2}+1}\right\}, \\
& \cos ^{2} \frac{\xi_{2}}{2}=\frac{1}{2}\left\{1+\left(2-e^{2 \theta}\right) k^{2}+\sqrt{\left(2-e^{2 \theta}\right)^{2} k^{4}-2\left(2 e^{-2 \theta}-\left(2-e^{2 \theta}\right)\right) k^{2}+1}\right\} .
\end{aligned}
$$

In the following, we consider the case that $\xi_{1}, \xi_{2} \in(0, \pi)$ so that $\sin \frac{\xi_{1}}{2}>0$ and $\sin \frac{\xi_{2}}{2}>0$, and define $\xi_{1}(k, \theta), \xi_{2}(k, \theta)$ by the equations (35), (36), (37) and (38). By a simple calculation, if $0<|k|<1$ and $0<\theta<\frac{1}{2} \log 2$, we have

$$
0<\frac{1}{2}\left\{1 \pm\left(2-e^{2 \theta}\right) k^{2} \pm \sqrt{\left(2-e^{2 \theta}\right)^{2} k^{4}-2\left(2 e^{-2 \theta}-\left(2-e^{2 \theta}\right)\right) k^{2}+1}\right\}<1 .
$$

Note that

$$
2-e^{2 \theta}>0
$$

if $0<\theta<\frac{1}{2} \log 2$.

We put

$$
\begin{aligned}
& a=a(\theta)=\sqrt{2-e^{2 \theta}}, \\
& b=b(\theta)=\frac{2 \sinh \theta}{\sqrt{2-e^{2 \theta}}} .
\end{aligned}
$$

Then we have

Lemma 4.1. Assume $0<\theta<\frac{1}{2} \log 2$. Then $\xi_{j}(k, \theta)$ has the analytic continuations $\zeta_{j}(z, \theta), j \in\{1,2\}$, with the following properties:

1. $\zeta_{j}(z, \theta), j \in\{1,2\}$, are analytic with respect to $z \in \mathbb{C}_{+}=\{z \in \mathbb{C} ; \Im z>0\}$.

2. If $k \in(-1,0) \cup(0,1)$, we have $\zeta_{j}(k+i 0, \theta)=\xi_{j}(k, \theta), j \in\{1,2\}$.

3. If $0<\xi_{j}<\pi, j \in\{1,2\}$, there exist $m_{1}, m_{2} \in \mathbb{Z}$ such that

$$
\begin{gathered}
\Re \zeta_{1}(1+i N, \theta)=2 m_{1} \pi+\mathcal{O}\left(N^{-3}\right), \\
\Im \zeta_{1}(1+i N, \theta)=2 \log \left(b+\sqrt{b^{2}+1}\right)+\mathcal{O}\left(N^{-2}\right), \\
\Re \zeta_{2}(1+i N, \theta)=\left(2 m_{2}+1\right) \pi+\mathcal{O}\left(N^{-1}\right), \\
\Im \zeta_{2}(1+i N, \theta)=2 \log N+\log \left(4 a^{2}\right)+\mathcal{O}\left(N^{-2}\right),
\end{gathered}
$$

as $N \rightarrow+\infty$.

Proof. We put

$$
f_{ \pm}(x)=\frac{1}{2}\left\{1-\left(2-e^{2 \theta}\right) x \pm \sqrt{\left(1-\left(2-e^{2 \theta}\right) x^{2}\right)^{2}-4^{2} x^{2} \sinh ^{2} \theta}\right\}
$$


for $x \geq 0$. Note that

$$
\begin{gathered}
0 \leq f_{ \pm}(x) \leq 1, \\
f_{-}(0)=0, f_{+}(0)=1, \\
\lim _{x \rightarrow+\infty} f_{-}(x)=1, \lim _{x \rightarrow+\infty} f_{+}(x)=0 .
\end{gathered}
$$

A simple calculation shows that $f_{-}(x)$ (resp. $f_{+}(x)$ ) are increasing (resp. decreasing) monotonically.

We also put

$$
\begin{aligned}
g(z) & =\left(1-\left(2-e^{2 \theta}\right) z^{2}\right)^{2}-4^{2} z^{2} \sinh ^{2} \theta \\
& =\left(2-e^{2 \theta}\right)^{2} z^{4}-2\left(2 e^{-2 \theta}-\left(2-e^{2 \theta}\right)\right) z^{2}+1
\end{aligned}
$$

Another simple calculation shows that all the zeros of $g(z)$ belong to $\mathbb{R}$. Then we have the analytic continuations of $f_{ \pm}\left(z^{2}\right)$ with respect to $z \in \mathbb{C}_{+}$.

Furthermore, another simple calculation shows that $0 \leq f_{ \pm}\left(z^{2}\right) \leq 1$ if and only if $z \in \mathbb{R}$. Therefore, we have the analytic continuations $\zeta_{j}(z, \theta)$ of $\xi_{j}(k, \theta)$, $j \in\{1,2\}, z \in \mathbb{C}_{+}$, which have the imaginary parts of fixed signs.

Let $|z| \rightarrow \infty$. Then we have the following asymptotic behaviors:

$$
\begin{gathered}
\sin ^{2} \frac{\zeta_{1}}{2}=-4 \frac{\sinh ^{2} \theta}{2-e^{2 \theta}}-4 \frac{e^{-2 \theta} \sinh ^{2} \theta}{\left(2-e^{2 \theta}\right)^{3}} z^{-2}+\mathcal{O}\left(|z|^{-4}\right) \\
\sin ^{2} \frac{\zeta_{2}}{2}=-\left(2-e^{2 \theta}\right) z^{2}+\frac{e^{-2 \theta}}{2-e^{2 \theta}}+4 \frac{e^{-2 \theta} \sinh ^{2} \theta}{\left(2-e^{2 \theta}\right)^{3}} z^{-2}+\mathcal{O}\left(|z|^{-4}\right), \\
\cos ^{2} \frac{\zeta_{1}}{2}=\frac{e^{-2 \theta}}{2-e^{2 \theta}}+4 \frac{e^{-2 \theta} \sinh ^{2} \theta}{\left(2-e^{2 \theta}\right)^{3}} z^{-2}+\mathcal{O}\left(|z|^{-4}\right) \\
\cos ^{2} \frac{\zeta_{2}}{2}=\left(2-e^{2 \theta}\right) z^{2}-4 \frac{\sinh ^{2} \theta}{2-e^{2 \theta}}-4 \frac{e^{-2 \theta} \sinh ^{2} \theta}{\left(2-e^{2 \theta}\right)^{3}} z^{-2}+\mathcal{O}\left(|z|^{-4}\right) .
\end{gathered}
$$

Note that $\sin \frac{\xi_{j}}{2}>0$ and $\cos \frac{\xi_{j}}{2}>0$, since $0<\xi_{j}<\pi, j \in\{1,2\}$. Therefore, we have

$$
\begin{aligned}
& \sin \frac{\zeta_{1}}{2}=i \frac{2 \sinh \theta}{\sqrt{2-e^{2 \theta}}}\left\{1+\frac{e^{-2 \theta}}{2\left(2-e^{2 \theta}\right)^{2}} z^{-2}+\mathcal{O}\left(|z|^{-4}\right)\right\}, \\
& \sin \frac{\zeta_{2}}{2}=i \sqrt{2-e^{2 \theta}} z\left\{1-\frac{e^{-2 \theta}}{2\left(2-e^{2 \theta}\right)^{2}} z^{-2}+\mathcal{O}\left(|z|^{-4}\right)\right\}, \\
& \cos \frac{\zeta_{1}}{2}=\frac{e^{-\theta}}{\sqrt{2-e^{2 \theta}}}\left\{1+2 \frac{\sinh ^{2} \theta}{\left(2-e^{2 \theta}\right)^{2}} z^{-2}+\mathcal{O}\left(|z|^{-4}\right)\right\}, \\
& \cos \frac{\zeta_{2}}{2}=\sqrt{2-e^{2 \theta}} z\left\{1-2 \frac{\sinh ^{2} \theta}{\left(2-e^{2 \theta}\right)^{2}} z^{-2}+\mathcal{O}\left(|z|^{-4}\right)\right\} .
\end{aligned}
$$


Let $E_{j}=E_{j}(z)$ be the analytic continuations of $\xi_{j}, j \in\{1,2\}$. We put $z=1+i N$. Then, from (49) and (52), we have

$$
\begin{aligned}
& \sin \frac{E_{1}(z)}{2}=x_{s, 1}+i y_{s, 1}, \\
& \cos \frac{E_{2}(z)}{2}=x_{c, 2}+i y_{c, 2},
\end{aligned}
$$

where

$$
\begin{gathered}
x_{s, 1}=b(\theta)\left\{\frac{e^{-2 \theta}}{\left(2-e^{2 \theta}\right)^{2}} N^{-3}+\mathcal{O}\left(N^{-5}\right)\right\}, \\
y_{s, 1}=b(\theta)\left\{1-\frac{e^{-2 \theta}}{2\left(2-e^{2 \theta}\right)^{2}} N^{-2}+\mathcal{O}\left(N^{-4}\right)\right\}, \\
x_{c, 2}=a(\theta)\left\{1-2 \frac{\sinh ^{2} \theta}{\left(2-e^{2 \theta}\right)^{2}} N^{-2}+\mathcal{O}\left(N^{-4}\right)\right\}, \\
y_{c, 2}=a(\theta)\left\{N+2 \frac{\sinh ^{2} \theta}{\left(2-e^{2 \theta}\right)^{2}} N^{-1}+\mathcal{O}\left(N^{-3}\right)\right\},
\end{gathered}
$$

and $a=a(\theta)$ and $b=b(\theta)$ is defined by (39) and (40), respectively. We also put $E_{j}=\eta_{j}+i \kappa_{j}, j \in\{1,2\}$. Then, from (53) and (54), we have

$$
\begin{aligned}
& \sin \frac{\eta_{1}}{2} \cosh \frac{\kappa_{1}}{2}=x_{s, 1}, \\
& \cos \frac{\eta_{1}}{2} \sinh \frac{\kappa_{1}}{2}=y_{s, 1}, \\
& \cos \frac{\eta_{2}}{2} \cosh \frac{\kappa_{2}}{2}=x_{c, 2}, \\
& -\sin \frac{\eta_{2}}{2} \sinh \frac{\kappa_{2}}{2}=y_{c, 2} .
\end{aligned}
$$

For sufficiently large $N>0$, from (55) and (57), we have $x_{s, 1}>0$ and $x_{c, 2}>0$, which implies, from (59) and (61), that $\sin \frac{\eta_{1}}{2}>0$ and $\cos \frac{\eta_{2}}{2}>0$, since $\cosh \frac{\kappa_{j}}{2}>0, j \in\{1,2\}$. We can now prove (63), (64), (65), and (66) in the same way as in Isozaki and Korotyaev [9] by carefully investigating the asymptotic behaviors as $N \rightarrow+\infty$. We shall give the proof of (63) and (64) in B.1 and that of (63) and (64) in B.2. respectively.

Remark 4.1. In the case that $\xi_{1}, \xi_{2} \in(-\pi, 0)$, we have $\sin \frac{\xi_{j}}{2}<0$ and $\cos \frac{\xi_{j}}{2}>$ $0, j \in\{1,2\}$. Therefore, also in this case, we can prove the same lemma as Lemma 4.1. where we replace the third statement in Lemma 4.1 with the following one: 
3' If $-\pi<\xi_{j}<0, j \in\{1,2\}$, there exist $m_{3}, m_{4} \in \mathbb{Z}$ such that

$$
\begin{gathered}
\Re \zeta_{1}(1+i N, \theta)=2 m_{3} \pi+\mathcal{O}\left(N^{-3}\right), \\
\Im \zeta_{1}(1+i N, \theta)=-2 \log \left(b+\sqrt{b^{2}+1}\right)+\mathcal{O}\left(N^{-2}\right), \\
\Re \zeta_{2}(1+i N, \theta)=\left(2 m_{4}+1\right) \pi+\mathcal{O}\left(N^{-1}\right), \\
\Im \zeta_{2}(1+i N, \theta)=-2 \log N-\log \left(4 a^{2}\right)+\mathcal{O}\left(N^{-2}\right),
\end{gathered}
$$

as $N \rightarrow+\infty$.

\section{Resolvent estimates}

We define the following non-negative quantities: for $n=\left(n_{1}, n_{2}\right) \in \mathbb{Z}^{2}$,

$$
\begin{gathered}
d(n)=d_{11}(n)=d_{22}(n)= \begin{cases}\left|n_{1}\right|+\left|n_{2}\right|, & \text { if } n_{1} \cdot n_{2} \geq 0, \\
\max \left\{\left|n_{1}\right|,\left|n_{2}\right|\right\}, & \text { if } n_{1} \cdot n_{2} \leq 0,\end{cases} \\
d_{12}(n)=d_{21}(-n)= \begin{cases}\left|n_{1}\right|+\left|n_{2}\right|-1, & \text { if } n_{1}>0, n_{2}>0, \\
\max \left\{\left|n_{1}\right|-1,\left|n_{2}\right|\right\}, & \text { if } n_{1}>0, n_{2} \leq 0, \\
\left|n_{1}\right|+\left|n_{2}\right|, & \text { if } n_{1} \leq 0, n_{2} \leq 0, \\
\max \left\{\left|n_{1}\right|,\left|n_{2}\right|-1\right\}, & \text { if } n_{1} \leq 0, n_{2}>0 .\end{cases}
\end{gathered}
$$

The contour lines of $d_{i j}(\cdot), i, j \in\{1,2\}$, are shown in Figure 4. The quantity $d(\cdot)$ is used to estimate the support of the Fourier coefficients of $|\alpha(\xi)|^{2}$ in Lemma 5.6. where $\alpha(\xi)$ is defined by (3). Also, $d_{i j}(\cdot),(i, j)=(1,2)$ or $(2,1)$, are used for $|\alpha(\xi)|^{2} \alpha(\xi)$ or $|\alpha(\xi)|^{2} \bar{\alpha}(\xi)$ in Lemmas 5.7 or 5.8 respectively. By making use of those Lemmas, we can derive the resolvent estimates at the end this section.
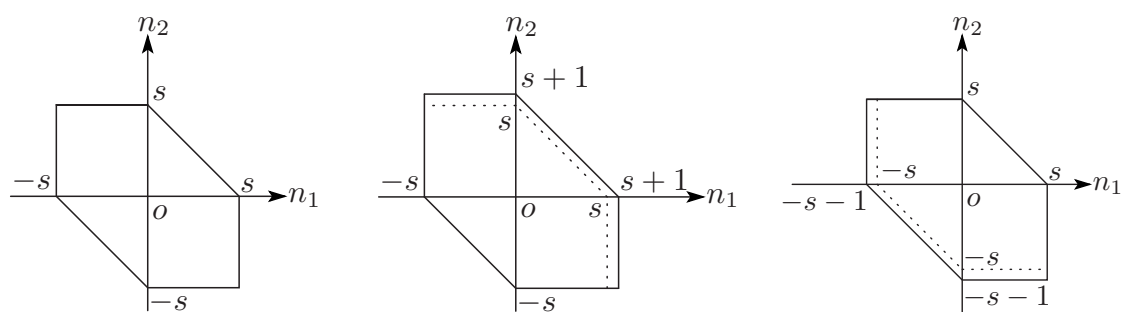

Figure 4: The shape of contour lines of $d_{i j}(n)=s ;(i, j)=(1,1)$ or $(2,2),(1,2)$, and $(2,1)$ from the left to the right.

The following two lemmas are immediate consequences from the definitions (67) and 68). 
Lemma 5.1. For $n=\left(n_{1}, n_{2}\right) \in \mathbb{Z}^{2}$,

$$
\begin{gathered}
d(n) \geq\left|n_{2}\right|, \\
d_{12}(n) \geq \begin{cases}\left|n_{2}\right|-1, & \text { if } n_{2}>0, \\
\left|n_{2}\right|, & \text { if } n_{2} \leq 0,\end{cases} \\
d_{21}(n) \geq \begin{cases}\left|n_{2}\right|, & \text { if } n_{2} \geq 0, \\
\left|n_{2}\right|-1, & \text { if } n_{2}<0 .\end{cases}
\end{gathered}
$$

Lemma 5.2. For $i, j \in\{1,2\}$,

$$
d(n)-1 \leq d_{i j}(n) \leq d(n) .
$$

We can easily show that $d(a n)=|a| d(n)$ for $a \in \mathbb{Z}$; also, that $d(n)=0$ if and only if $n=(0,0)$. Moreover, we have

Lemma 5.3. $d(\cdot)$ is a norm on $\mathbb{Z}^{2}$. In particular, it satisfies the triangle inequality:

$$
d(m-n) \leq d(m-l)+d(l-n) .
$$

Proof. We only have to prove (69). Assume that $m_{1}-n_{1} \geq 0$ and $m_{2}-n_{2} \geq 0$, where $m=\left(m_{1}, m_{2}\right), n=\left(n_{1}, n_{2}\right) \in \mathbb{Z}^{2}$. We treat the following nine cases according to the locations of $l=\left(l_{1}, l_{2}\right) \in \mathbb{Z}^{2}$ :

(1) $l_{1}-n_{1} \geq 0, l_{2}-n_{2} \geq 0, m_{1}-l_{1} \geq 0, m_{2}-l_{2} \geq 0$

(2) $l_{1}-n_{1} \geq 0, l_{2}-n_{2} \geq 0, m_{1}-l_{1} \geq 0, m_{2}-l_{2} \leq 0$,

(3) $l_{1}-n_{1} \geq 0, l_{2}-n_{2} \geq 0, m_{1}-l_{1} \leq 0, m_{2}-l_{2} \geq 0$,

(4) $l_{1}-n_{1} \geq 0, l_{2}-n_{2} \geq 0, m_{1}-l_{1} \leq 0, m_{2}-l_{2} \leq 0$,

(5) $l_{1}-n_{1} \leq 0, l_{2}-n_{2} \geq 0, m_{1}-l_{1} \geq 0, m_{2}-l_{2} \geq 0$,

(6) $l_{1}-n_{1} \leq 0, l_{2}-n_{2} \geq 0, m_{1}-l_{1} \geq 0, m_{2}-l_{2} \leq 0$,

(7) $l_{1}-n_{1} \geq 0, l_{2}-n_{2} \leq 0, m_{1}-l_{1} \geq 0, m_{2}-l_{2} \geq 0$,

(8) $l_{1}-n_{1} \geq 0, l_{2}-n_{2} \leq 0, m_{1}-l_{1} \leq 0, m_{2}-l_{2} \geq 0$,

(9) $l_{1}-n_{1} \leq 0, l_{2}-n_{2} \leq 0, m_{1}-l_{1} \leq 0, m_{2}-l_{2} \leq 0$.

The left figure in Figure 5 illustrates the location of $l \in \mathbb{Z}^{2}$ which corresponds to the above nine cases.

Let us consider, for example, the case (3). Then we have

$$
\begin{aligned}
d(m-n) & =\left|m_{1}-n_{1}\right|+\left|m_{2}-n_{2}\right| \\
& =\left(m_{1}-l_{1}\right)+\left(l_{1}-n_{1}\right)+\left|m_{2}-n_{2}\right| .
\end{aligned}
$$



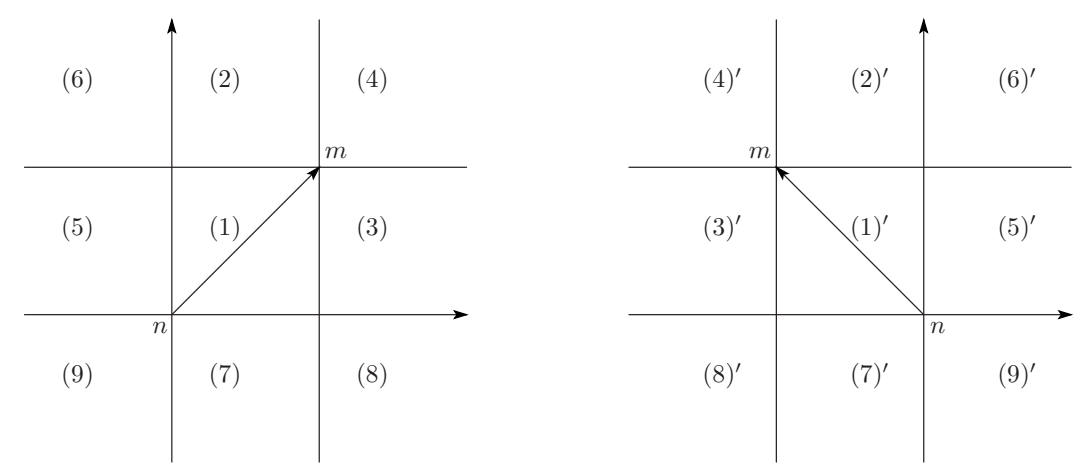

Figure 5: The left figure shows the location of $l \in \mathbb{Z}^{2}$ corresponding to the case from (11) to (9); the right one corresponds that of $l \in \mathbb{Z}^{2}$ from (1) $)^{\prime}$ to (9)'.

By the assumption, we have $m_{1}-l_{1} \leq 0$, which implies

$$
\begin{aligned}
d(m-n) & \leq\left|l_{1}-n_{1}\right|+\left|m_{2}-l_{2}\right|+\left|l_{2}-n_{2}\right| \\
& \leq \max \left\{\left|m_{1}-l_{1}\right|,\left|m_{2}-l_{2}\right|\right\}+\left|l_{1}-n_{1}\right|+\left|l_{2}-n_{2}\right| \\
& =d(m-l)+d(l-n) .
\end{aligned}
$$

We can treat the other cases in the same way.

Next, assume that $m_{1}-n_{1} \leq 0$ and $m_{2}-n_{2} \geq 0$. This time, we treat the following nine cases according to the location of $l$ :

$(1)^{\prime} l_{1}-n_{1} \leq 0, l_{2}-n_{2} \geq 0, m_{1}-l_{1} \leq 0, m_{2}-l_{2} \geq 0$,

$(2)^{\prime} l_{1}-n_{1} \leq 0, l_{2}-n_{2} \geq 0, m_{1}-l_{1} \leq 0, m_{2}-l_{2} \leq 0$,

$(3)^{\prime} l_{1}-n_{1} \leq 0, l_{2}-n_{2} \geq 0, m_{1}-l_{1} \geq 0, m_{2}-l_{2} \geq 0$,

$(4)^{\prime} l_{1}-n_{1} \leq 0, l_{2}-n_{2} \geq 0, m_{1}-l_{1} \geq 0, m_{2}-l_{2} \leq 0$,

$(5)^{\prime} l_{1}-n_{1} \geq 0, l_{2}-n_{2} \geq 0, m_{1}-l_{1} \leq 0, m_{2}-l_{2} \geq 0$,

$(6)^{\prime} l_{1}-n_{1} \geq 0, l_{2}-n_{2} \geq 0, m_{1}-l_{1} \leq 0, m_{2}-l_{2} \leq 0$,

$(7)^{\prime} l_{1}-n_{1} \leq 0, l_{2}-n_{2} \leq 0, m_{1}-l_{1} \leq 0, m_{2}-l_{2} \geq 0$,

$(8)^{\prime} l_{1}-n_{1} \leq 0, l_{2}-n_{2} \leq 0, m_{1}-l_{1} \geq 0, m_{2}-l_{2} \geq 0$,

$(9)^{\prime} l_{1}-n_{1} \geq 0, l_{2}-n_{2} \leq 0, m_{1}-l_{1} \leq 0, m_{2}-l_{2} \geq 0$.

The right figure in Figure 5 illustrates the location of $l \in \mathbb{Z}^{2}$ which corresponds to the above nine cases.

Let us consider, for example, the case (3)'. Then we have

$$
\begin{aligned}
d(m-n) & =\max \left\{\left|m_{1}-n_{1}\right|,\left|m_{2}-n_{2}\right|\right\} \\
& =\max \left\{\left(n_{1}-l_{1}\right)+\left(l_{1}-m_{1}\right),\left|m_{2}-n_{2}\right|\right\} .
\end{aligned}
$$


By the assumption, we have $l_{1}-m_{1} \leq 0$, which implies

$$
\begin{aligned}
d(m-n) & \leq \max \left\{\left|l_{1}-n_{1}\right|,\left|m_{2}-l_{2}\right|+\left|l_{2}-n_{2}\right|\right\} \\
& \leq\left|m_{2}-l_{2}\right|+\max \left\{\left|l_{1}-n_{1}\right|,\left|l_{2}-n_{2}\right|\right\} \\
& \leq\left|m_{1}-l_{2}\right|+\left|m_{2}-l_{2}\right|+\max \left\{\left|l_{1}-n_{1}\right|,\left|l_{2}-n_{2}\right|\right\} \\
& =d(m-l)+d(l-n) .
\end{aligned}
$$

We can treat the other cases in the same way.

The case that $m_{1}-n_{1} \leq 0$ and $m_{2}-n_{2} \leq 0$ can be reduced to the first one; the remaining one to the second.

Remark 5.1. One can convince oneself of the above lemma if one bears the shape of the contour lines of $d_{i j}(\cdot)$ in mind and shifts them. One can also have insight into Lemmas 5.4 and 5.5 in the same way.

We can prove the following two lemmas in the same way as above, whose proofs will be given in Appendix C

Lemma 5.4. For $i, j \in\{1,2\}$,

$$
\begin{aligned}
& d_{i j}(m-n) \leq d_{i i}(m-l)+d_{i j}(l-n), \\
& d_{i j}(m-n) \leq d_{i j}(m-l)+d_{j j}(l-n) .
\end{aligned}
$$

Lemma 5.5. For $i, j \in\{1,2\}$,

$$
d_{i i}(m-n) \leq d_{i j}(m-l)+d_{j i}(l-n)+1 .
$$

We put $r(\xi)=|\alpha(\xi)|^{2}$, where $\alpha(\xi)$ is defined in (3). Then we have

Lemma 5.6. Let $s \in \mathbb{Z}, s \geq 0$. Then the Fourier coefficients of $r(\xi)^{s}$ are supported on the set $\left\{n \in \mathbb{Z}^{2} ; d(n) \leq s\right\}$, that is,

$$
\int_{\mathbb{T}^{2}} r(\xi)^{s} e^{-i n \xi} d \xi=0
$$

when $d(n)>s$.

Proof. The claim in the lemma is obvious if $s=0$.

By the definition of $r(\xi)$, we have

$$
r(\xi)=3+e^{i \xi_{1}}+e^{-i \xi_{1}}+e^{i \xi_{2}}+e^{-i \xi_{2}}+e^{i\left(\xi_{1}-\xi_{2}\right)}+e^{-i\left(\xi_{1}-\xi_{2}\right)},
$$

which means that $r(\xi)$ is a linear combination of $e^{i n_{0} \xi}, d\left(n_{0}\right) \leq 1$.

Assume that we have proved the claim for $s \leq s_{0}$. Then $r(\xi)^{s_{0}}$ is a linear combination of $e^{i n \xi}, d(n) \leq s_{0}$. Therefore, $r(\xi)^{s_{0}+1}$ is a linear combination of $e^{i n \xi} r(\xi), d(n) \leq s_{0}$, which means, from (72), that it is that of $e^{i m \xi}, m=n+n_{0}$, where $d(n) \leq s_{0}$ and $d\left(n_{0}\right) \leq 1$. By using the triangle inequality (69), we have

$$
\begin{aligned}
d(m) & =d\left(n+n_{0}\right) \\
& \leq d(n)+d\left(n_{0}\right) \\
& \leq s_{0}+1,
\end{aligned}
$$


which proves the lemma by the induction argument with respect to $s \in \mathbb{Z}$, $s \geq 0$.

In a similar way, we have

Lemma 5.7. Let $s \in \mathbb{Z}, s \geq 0$. Then the Fourier coefficients of $r(\xi)^{s} \alpha(\xi)$ are supported on the set $\left\{n \in \mathbb{Z}^{2} ; d_{12}(n) \leq s\right\}$, that is,

$$
\int_{\mathbb{T}^{2}} r(\xi)^{s} \alpha(\xi) e^{-i n \xi} d \xi=0
$$

when $d_{12}(n)>s$.

Proof. We have already proved that $r(\xi)^{s}$ is a linear combination of $e^{i n \xi}, d(n) \leq$ $s$. From (3),$\alpha(\xi)$ is a sum of $e^{i n_{0} \xi}$, where $n_{0} \in\{(0,0),(1,0),(0,1)\}$. Therefore, $r(\xi)^{s} \alpha(\xi)$ is a linear combination of $e^{i n \xi} \alpha(\xi), d(n) \leq s$, which means that it is that of $e^{i m \xi}, m=n+n_{0}$, where $d(n) \leq s$ and $n_{0} \in\{(0,0),(1,0),(0,1)\}$. By the definition (68), we have $d_{12}(m) \leq s$ (see also the shape of the contour line of $d_{12}(\cdot)$ in Figure 4), which proves the lemma.

Noticing that

$$
\overline{\int_{\mathbb{T}^{2}} r(\xi)^{s} \alpha(\xi) e^{-i n \xi} d \xi}=\int_{\mathbb{T}^{2}} r(\xi)^{s} \bar{\alpha}(\xi) e^{i n \xi} d \xi
$$

and that $d_{12}(n)=d_{21}(-n)$, we have

Lemma 5.8. Let $s \in \mathbb{Z}, s \geq 0$. Then the Fourier coefficients of $r(\xi)^{s} \bar{\alpha}(\xi)$ are supported on the set $\left\{n \in \mathbb{Z}^{2} ; d_{21}(n) \leq s\right\}$, that is,

$$
\int_{\mathbb{T}^{2}} r(\xi)^{s} \bar{\alpha}(\xi) e^{-i n \xi} d \xi=0
$$

when $d_{21}(n)>s$.

Now, let us investigate the asymptotic behavior of the resolvents as $|z| \rightarrow \infty$. We begin with $\hat{R}_{0}(z)$. We will also use $\hat{P}(n)$ as the projection onto the site $n \in \mathbb{Z}^{2}$ on $l^{2}\left(\mathbb{Z}^{2}\right)$.

Lemma 5.9. If $\hat{f}=\left(\hat{f}_{1}, \hat{f}_{2}\right) \in l^{2}\left(\mathbb{Z}^{2} ; \mathbb{C}^{2}\right)$ is compactly supported, then we have

$$
\hat{R}_{0}(z) \hat{f}=\left(\begin{array}{l}
\sum_{s=0}^{\infty} z^{-2 s-1} \hat{R}_{0, s, 11} \hat{f}_{1}+\sum_{s=0}^{\infty} z^{-2 s-2} \hat{R}_{0, s, 12} \hat{f}_{2} \\
\sum_{s=0}^{\infty} z^{-2 s-2} \hat{R}_{0, s, 21} \hat{f}_{1}+\sum_{s=0}^{\infty} z^{-2 s-1} \hat{R}_{0, s, 22} \hat{f}_{2}
\end{array}\right)
$$

for sufficiently large $|z|$, where $\hat{R}_{0, s, i j} \in \mathbf{B}\left(l^{2}\left(\mathbb{Z}^{2}\right)\right)$ with the properties

$$
\hat{P}(m) \hat{R}_{0, s, i j}(z) \hat{P}(n)=0,
$$

if $d_{i j}(m-n)>s, i, j \in\{1,2\}$. 
Proof. From (28) and (29), we have

$$
\left(\hat{R}_{0, s, i j} \hat{f}_{j}\right)(n)=\sum_{m \in \mathbb{Z}^{2}} \hat{r}_{0, s, i j}(n-m) \hat{f}_{j}(m)
$$

where

$$
\begin{gathered}
\hat{r}_{0, s, 11}(k)=\hat{r}_{0, s, 22}(k)=\int_{\mathbb{T}^{2}} r(\xi)^{s} e^{-i k \xi} d \xi, \\
\hat{r}_{0, s, 12}(k)=\int_{\mathbb{T}^{2}} r(\xi)^{s} \alpha(\xi) e^{-i k \xi} d \xi \\
\hat{r}_{0, s, 21}(k)=\int_{\mathbb{T}^{2}} r(\xi)^{s} \bar{\alpha}(\xi) e^{-i k \xi} d \xi .
\end{gathered}
$$

Lemmas 5.6, 5.7, and 5.8 imply the above properties of $\hat{R}_{0, s, i j}, i, j \in\{1,2\}$, which proves the lemma.

Next, we consider the asymptotic behavior of $\hat{R}(z)$ for large $|z|$.

Lemma 5.10. For sufficiently large $|z|$,

$$
\hat{P}(m) \hat{R}(z) \hat{P}(n)=\left(\begin{array}{cc}
\mathcal{O}\left(\langle z\rangle^{-2 d(m-n)-1}\right) & \mathcal{O}\left(\langle z\rangle^{-2 d_{12}(m-n)-2}\right) \\
\mathcal{O}\left(\langle z\rangle^{-2 d_{21}(m-n)-2}\right) & \mathcal{O}\left(\langle z\rangle^{-2 d(m-n)-1}\right)
\end{array}\right) .
$$

Proof. Let $p=d(m-n)$. Then, by using the resolvent equation repeatedly, we have the following expansion:

$$
\hat{R}(z)=\hat{R}_{0}(z)-\hat{R}_{0}(z) \hat{q} \hat{R}_{0}(z)+\cdots+(-1)^{2 p-1} \underbrace{\hat{R}_{0}(z) \hat{q} \hat{R}_{0}(z) \cdots \hat{q} \hat{R}_{0}(z)}_{2 p \hat{R}_{0}(z) \text { 's and } 2 p-1 \hat{q}^{\prime} \mathbf{s}}+\mathcal{O}\left(\langle z\rangle^{-2 p-1}\right) \text {. }
$$

By multiplying $\hat{P}(m)$ and $\hat{P}(n)$ from the left and the right of (74), respectively, we have

$$
\hat{P}(m) \hat{R}_{0}(z) \hat{P}(n)=\left(\begin{array}{ll}
\sum_{s=0}^{\infty} z^{-2 z-1} \hat{P}(m) \hat{R}_{0, s, 11} \hat{P}(n) & \sum_{s=0}^{\infty} z^{-2 z-2} \hat{P}(m) \hat{R}_{0, s, 12} \hat{P}(n) \\
\sum_{s=0}^{\infty} z^{-2 z-2} \hat{P}(m) \hat{R}_{0, s, 21} \hat{P}(n) & \sum_{s=0}^{\infty} z^{-2 z-1} \hat{P}(m) \hat{R}_{0, s, 22} \hat{P}(n)
\end{array}\right)
$$

as the fist term of the right hand side. Then, from Lemma 5.9 we have

$$
\begin{aligned}
\sum_{s=0}^{\infty} z^{-2 z-1} \hat{P}(m) \hat{R}_{0, s, i i} \hat{P}(n) & =\sum_{s \geq d(m-n)} z^{-2 z-1} \hat{P}(m) \hat{R}_{0, s, i i} \hat{P}(n) \\
& =\mathcal{O}\left(\langle z\rangle^{-2 d(m-n)-1}\right), \\
\sum_{s=0}^{\infty} z^{-2 z-2} \hat{P}(m) \hat{R}_{0, s, i j} \hat{P}(n) & =\sum_{s \geq d_{i j}(m-n)} z^{-2 z-2} \hat{P}(m) \hat{R}_{0, s, i j} \hat{P}(n) \\
& =\mathcal{O}\left(\langle z\rangle^{-2 d_{i j}(m-n)-2}\right),
\end{aligned}
$$


for $(i, j)=(1,2)$ or $(2,1)$.

About the $k$-th term, $1 \leq k \leq p$, we use inductions. Such a term is written as a sum of

$$
\hat{P}(m) \hat{R}_{0}(z) \hat{P}\left(l^{(1)}\right) \hat{q}\left(l^{(1)}\right) \hat{P}\left(l^{(1)}\right) \hat{R}_{0}(z) \cdots \hat{P}\left(l^{(k)}\right) \hat{q}\left(l^{(k)}\right) \hat{P}\left(l^{(k)}\right) \hat{R}_{0}(z) \hat{P}(n),
$$

since $\hat{q}=\sum_{\sharp\left\{l \in \mathbb{Z}^{2}\right\}<\infty} \hat{P}(l) \hat{q}(l) \hat{P}(l)$.

Assume that (75) is estimated as (73) for $k=k_{0}-1$. Then, by the hypothesis of the induction, we have

$$
\begin{aligned}
\hat{P}(m) & \hat{R}_{0}(z) \hat{P}\left(l^{(1)}\right) \hat{q}\left(l^{(1)}\right) \hat{P}\left(l^{(1)}\right) \hat{R}_{0}(z) \cdots \hat{P}\left(l^{\left(k_{0}-1\right)}\right) \hat{q}\left(l^{\left(k_{0}-1\right)}\right) \hat{P}\left(l^{\left(k_{0}-1\right)}\right) \hat{R}_{0}(z) \hat{P}\left(l^{\left(k_{0}\right)}\right) \\
& \cdot \hat{q}\left(l^{\left(k_{0}\right)}\right) \cdot \hat{P}\left(l^{\left(k_{0}\right)}\right) \hat{R}_{0}(z) \hat{P}(n) \\
= & \left(\begin{array}{cc}
\mathcal{O}\left(\langle z\rangle^{-2 d\left(m-l^{\left(k_{0}\right)}\right)-1}\right) & \mathcal{O}\left(\langle z\rangle^{-2 d_{12}\left(m-l^{\left(k_{0}\right)}\right)-2}\right) \\
\mathcal{O}\left(\langle z\rangle^{-2 d_{21}\left(m-l^{\left(k_{0}\right)}\right)-2}\right) & \mathcal{O}\left(\langle z\rangle^{-2 d\left(m-l^{\left(k_{0}\right)}\right)-1}\right)
\end{array}\right) \\
& \cdot\left(\begin{array}{cc}
\hat{q}_{1}\left(l^{\left(k_{0}\right)}\right) & 0 \\
0 & \hat{q}_{2}\left(l^{\left(k_{0}\right)}\right)
\end{array}\right) \cdot\left(\begin{array}{cc}
\mathcal{O}\left(\langle z\rangle^{-2 d\left(l^{\left(k_{0}\right)}-n\right)-1}\right) & \mathcal{O}\left(\langle z\rangle^{-2 d_{12}\left(l^{\left(k_{0}\right)}-n\right)-2}\right) \\
\mathcal{O}\left(\langle z\rangle^{-2 d_{21}\left(l^{\left(k_{0}\right)}-n\right)-2}\right) & \mathcal{O}\left(\langle z\rangle^{-2 d\left(l^{\left(k_{0}\right)}-n\right)-1}\right)
\end{array}\right),
\end{aligned}
$$

which reads, from Lemmas 5.3, 5.4, and 5.5, that the upper left element is

$$
\begin{aligned}
& \mathcal{O}\left(\langle z\rangle^{-2 d\left(m-l^{\left(k_{0}\right)}\right)-2 d\left(l^{\left(k_{0}\right)}-n\right)-2}\right)+\mathcal{O}\left(\langle z\rangle^{-2 d_{12}\left(m-l^{\left(k_{0}\right)}\right)-2 d_{21}\left(l^{\left(k_{0}\right)}-n\right)-4}\right) \\
& =\mathcal{O}\left(\langle z\rangle^{-2 d(m-n)-2}\right),
\end{aligned}
$$

and that the upper right one is

$$
\begin{aligned}
& \mathcal{O}\left(\langle z\rangle^{-2 d\left(m-l^{\left(k_{0}\right)}\right)-2 d_{12}\left(l^{\left(k_{0}\right)}-n\right)-3}\right)+\mathcal{O}\left(\langle z\rangle^{-2 d_{12}\left(m-l^{\left(k_{0}\right)}\right)-2 d\left(l^{\left(k_{0}\right)}-n\right)-3}\right) \\
& =\mathcal{O}\left(\langle z\rangle^{-2 d_{12}(m-n)-3}\right) .
\end{aligned}
$$

We also have the lower left and right elements as desired, which proves the lemma.

Remark 5.2. A more detailed argument in the induction above gives us the estimate (73) for the non-diagonal $\hat{q}(n)$ 's.

\section{Proof of the main theorem}

We will essentially follow the idea of Isozaki and Korotyaev 9]. Assume that $\lambda>0$; for $\lambda<0$, we can argue in the same way. Put

$$
B\left(k, \theta, \theta^{\prime}\right)=4(2 \pi)^{2}\left(J(k, \theta) J\left(k, \theta^{\prime}\right)\right)^{-1} A\left(k, \theta, \theta^{\prime}\right),
$$

where $A\left(k, \theta, \theta^{\prime}\right)$ is the integral kernel of the scattering amplitude defined in Section 3.3. Then we have $B\left(k, \theta, \theta^{\prime}\right)=B_{0}\left(k, \theta, \theta^{\prime}\right)-B_{1}\left(k, \theta, \theta^{\prime}\right)$, where

$$
\begin{aligned}
B_{0}\left(k, \theta, \theta^{\prime}\right) & =4(2 \pi)^{2}\left(J(k, \theta) J\left(k, \theta^{\prime}\right)\right)^{-1} \hat{\mathcal{F}}_{0}(\lambda) \hat{q} \hat{\mathcal{F}}_{0}(\lambda)^{*} \\
& =\left(\begin{array}{ll}
B_{0,11}\left(k, \theta, \theta^{\prime}\right) & B_{0,12}\left(k, \theta, \theta^{\prime}\right) \\
B_{0,21}\left(k, \theta, \theta^{\prime}\right) & B_{0,22}\left(k, \theta, \theta^{\prime}\right)
\end{array}\right),
\end{aligned}
$$




$$
\begin{aligned}
& B_{0,11}\left(k, \theta, \theta^{\prime}\right)=\sum_{n \in \mathbb{Z}^{2}} e^{i n\left(\xi(k, \theta)-\xi\left(k, \theta^{\prime}\right)\right)}\left(\hat{q}_{1}(n)+\frac{\alpha(\xi(k, \theta))}{\sqrt{8 k^{2}+1}} \frac{\bar{\alpha}\left(\xi\left(k, \theta^{\prime}\right)\right)}{\sqrt{8 k^{2}+1}} \hat{q}_{2}(n)\right), \\
& B_{0,22}\left(k, \theta, \theta^{\prime}\right)=\sum_{n \in \mathbb{Z}^{2}} e^{i n\left(\xi(k, \theta)-\xi\left(k, \theta^{\prime}\right)\right)}\left(\frac{\bar{\alpha}(\xi(k, \theta))}{\sqrt{8 k^{2}+1}} \frac{\alpha\left(\xi\left(k, \theta^{\prime}\right)\right)}{\sqrt{8 k^{2}+1}} \hat{q}_{1}(n)+\hat{q}_{2}(n)\right)
\end{aligned}
$$

( $B_{0,12}$ and $B_{0,21}$ are omitted), and

$$
\begin{aligned}
B_{1}\left(k, \theta, \theta^{\prime}\right)= & 4(2 \pi)^{2}\left(J(k, \theta) J\left(k, \theta^{\prime}\right)\right)^{-1} \hat{\mathcal{F}}_{0}(\lambda) \hat{q} \hat{R}(\lambda+i 0) \hat{q} \hat{\mathcal{F}}_{0}(\lambda)^{*} \\
= & \left(\begin{array}{ll}
B_{1,11}\left(k, \theta, \theta^{\prime}\right) & B_{1,12}\left(k, \theta, \theta^{\prime}\right) \\
B_{1,21}\left(k, \theta, \theta^{\prime}\right) & B_{1,22}\left(k, \theta, \theta^{\prime}\right)
\end{array}\right), \\
B_{1,11}\left(k, \theta, \theta^{\prime}\right)= & \sum_{n \in \mathbb{Z}^{2}} e^{i n \xi(k, \theta)} \hat{q}_{1}(n)\left(\hat{R}(\lambda+i 0) \hat{q} \hat{\varphi}^{(0)}\left(k, \theta^{\prime}\right)\right)_{11}(n) \\
+ & \frac{\alpha(\xi(k, \theta))}{\sqrt{8 k^{2}+1}} \sum_{n \in \mathbb{Z}^{2}} e^{i n \xi(k, \theta)} \hat{q}_{2}(n)\left(\hat{R}(\lambda+i 0) \hat{q} \hat{\varphi}^{(0)}\left(k, \theta^{\prime}\right)\right)_{21}(n), \\
B_{1,22}\left(k, \theta, \theta^{\prime}\right)= & \frac{\bar{\alpha}(\xi(k, \theta))}{\sqrt{8 k^{2}+1}} \sum_{n \in \mathbb{Z}^{2}} e^{i n \xi(k, \theta)} \hat{q}_{1}(n)\left(\hat{R}(\lambda+i 0) \hat{q} \hat{\varphi}^{(0)}\left(k, \theta^{\prime}\right)\right)_{12}(n) \\
& +\sum_{n \in \mathbb{Z}^{2}} e^{i n \xi(k, \theta)} \hat{q}_{2}(n)\left(\hat{R}(\lambda+i 0) \hat{q} \hat{\varphi}^{(0)}\left(k, \theta^{\prime}\right)\right)_{22}(n)
\end{aligned}
$$

( $B_{1,12}$ and $B_{1,21}$ are omitted). Here we put

$$
\begin{aligned}
\hat{\varphi}^{(0)}\left(k, \theta^{\prime}\right) & =2(2 \pi) J\left(k, \theta^{\prime}\right)^{-1} \hat{\psi}^{(0)}\left(k, \theta^{\prime}\right) \\
& =\left(\hat{\varphi}^{(0)}\left(n ; k, \theta^{\prime}\right)\right)_{n \in \mathbb{Z}^{2}},
\end{aligned}
$$

where

$$
\begin{aligned}
\hat{\varphi}^{(0)}\left(n ; k, \theta^{\prime}\right) & =2(2 \pi) J\left(k, \theta^{\prime}\right)^{-1} \hat{\psi}^{(0)}\left(n ; k, \theta^{\prime}\right) \\
& =e^{-i n \xi\left(k, \theta^{\prime}\right)} \hat{\eta}^{(0)}\left(k, \theta^{\prime}\right), n \in \mathbb{Z}^{2}, \\
\hat{\eta}^{(0)}\left(k, \theta^{\prime}\right) & =\left(\begin{array}{cc}
1 & \frac{\alpha\left(\xi\left(k, \theta^{\prime}\right)\right)}{\sqrt{8 k^{2}+1}} \\
\frac{\bar{\alpha}\left(\xi\left(k, \theta^{\prime}\right)\right)}{\sqrt{8 k^{2}+1}} & 1
\end{array}\right),
\end{aligned}
$$

and $\hat{\psi}\left(k, \theta^{\prime}\right)$ and $\hat{\psi}\left(n ; k, \theta^{\prime}\right)$ are defined by (22) and (23), respectively

Let $\zeta_{ \pm}(z, \theta)=\left(\zeta_{ \pm, 1}(z, \theta), \zeta_{ \pm, 2}(z, \theta)\right)$ be the analytic continuation of $\xi(k, \theta)=$ $\left(\xi_{1}(k, \theta), \xi_{2}(k, \theta)\right)$ in Lemma 4.1] Then $B_{0}\left(k, \theta, \theta^{\prime}\right)$ and $B_{1}\left(k, \theta, \theta^{\prime}\right)$ have the analytic continuations $B_{0}\left(z, \theta, \theta^{\prime}\right)$ and $B_{1}\left(z, \theta, \theta^{\prime}\right)$ for $z \in \mathbb{C}_{+}$, respectively, which are defined with $k$ replaced by $z, \xi(k, \theta)$ by $\zeta_{+}(z, \theta)$ and $\xi\left(k, \theta^{\prime}\right)$ by $\zeta_{-}\left(z, \theta^{\prime}\right)$. 
Let us take $-\pi<\xi_{j}(k, \theta)<0$ and $0<\xi_{j}\left(k, \theta^{\prime}\right)<\pi$ for $j \in\{1,2\}$. From Lemma 4.1, we have

$$
\begin{gathered}
e^{i n \zeta_{+}(z, \theta)} \sim N^{2 n_{2}} b_{1}(\theta)^{n_{1}} a_{1}(\theta)^{n_{2}}, \\
e^{-i n \zeta_{-}\left(z, \theta^{\prime}\right)} \sim N^{2 n_{2}} b_{1}\left(\theta^{\prime}\right)^{n_{1}} a_{1}\left(\theta^{\prime}\right)^{n_{2}}
\end{gathered}
$$

as $N \rightarrow \infty$, where $z=1+i N$ and

$$
\begin{gathered}
a_{1}(\theta)=(2 a(\theta))^{2}=4\left(2-e^{2 \theta}\right), \\
b_{1}(\theta)=\left(b(\theta)+\sqrt{b(\theta)^{2}+1}\right)^{2}=\frac{e^{2 \theta}}{2-e^{2 \theta}} .
\end{gathered}
$$

We also have

$$
\begin{gathered}
\frac{\alpha\left(\zeta_{+}(z, \theta)\right)}{\sqrt{8 z^{2}+1}}=\frac{1+e^{i \zeta_{+, 1}(z, \theta)}+e^{i \zeta_{+, 2}(z, \theta)}}{\sqrt{8 z^{2}+1}} \sim a_{2}(\theta) N+\mathcal{O}(1), \\
\frac{\bar{\alpha}\left(\zeta_{+}(z, \theta)\right)}{\sqrt{8 z^{2}+1}}=\frac{1+e^{-i \zeta_{+, 1}(z, \theta)}+e^{-i \zeta_{+, 2}(z, \theta)}}{\sqrt{8 z^{2}+1}} \sim b_{2}(\theta) N^{-1}+\mathcal{O}\left(N^{-2}\right), \\
\frac{\alpha\left(\zeta_{-}\left(z, \theta^{\prime}\right)\right)}{\sqrt{8 z^{2}+1}}=\frac{1+e^{i \zeta_{-, 1}(z, \theta)}+e^{i \zeta_{-, 2}(z, \theta)}}{\sqrt{8 z^{2}+1}} \sim b_{2}\left(\theta^{\prime}\right) N^{-1}+\mathcal{O}\left(N^{-2}\right), \\
\frac{\bar{\alpha}\left(\zeta_{-}\left(z, \theta^{\prime}\right)\right)}{\sqrt{8 z^{2}+1}}=\frac{1+e^{-i \zeta_{-, 1}(z, \theta)}+e^{-i \zeta_{-, 2}(z, \theta)}}{\sqrt{8 z^{2}+1}} \sim a_{2}\left(\theta^{\prime}\right) N+\mathcal{O}(1),
\end{gathered}
$$

where

$$
\begin{gathered}
a_{2}(\theta)=-\frac{a_{1}(\theta)}{2 \sqrt{2}} i=-\sqrt{2}\left(2-e^{2 \theta}\right) i, \\
b_{2}(\theta)=-\frac{1+b_{1}(\theta)^{-1}}{2 \sqrt{2}} i=-\frac{e^{-2 \theta}}{\sqrt{2}} i .
\end{gathered}
$$

By our assumption (A), the potential $\hat{q}$ is compactly supported; more precisely, for some $M \geq 0, M \in \mathbb{Z}$,

$$
|n|_{l^{1}}=\left|n_{1}\right|+\left|n_{2}\right|>M \Longrightarrow \hat{q}(n)=0 .
$$

We put

$$
n^{(M)}=(0, M) \in \mathbb{Z}^{2} .
$$

Then, by using (77), (78), (82), and (83), we have the following asymptotic expansion:

$$
B_{0,22}\left(z, \theta, \theta^{\prime}\right) \sim N^{4 M}\left(a_{1}(\theta) a_{1}\left(\theta^{\prime}\right)\right)^{4 M} \hat{q}_{2}\left(n^{(M)}\right) .
$$

Let us investigate the asymptotic behavior of $B_{1,22}\left(z, \theta, \theta^{\prime}\right)$. By introducing Dirac's notation, we have

$$
\hat{P}(m)=\left|\hat{p}_{m}\right\rangle\left\langle\hat{p}_{m}\right|,
$$

where

$$
\left(\hat{p}_{m}\right)(n)=\left(\begin{array}{l}
\delta_{m n} \\
\delta_{m n}
\end{array}\right) \in l^{2}\left(\mathbb{Z}^{2} ; \mathbb{C}^{2}\right) .
$$


From (83) and (84), we note that

$$
\hat{\eta}^{(0)}\left(z, \theta^{\prime}\right)=\left(\begin{array}{cc}
1 & \frac{\alpha\left(\zeta_{-}\left(z, \theta^{\prime}\right)\right)}{\sqrt{8 z^{2}+1}} \\
\frac{\bar{\alpha}\left(\zeta_{-}\left(z, \theta^{\prime}\right)\right)}{\sqrt{8 z^{2}+1}} & 1
\end{array}\right) \sim\left(\begin{array}{cc}
1 & \mathcal{O}\left(N^{-1}\right) \\
\mathcal{O}(N) & 1
\end{array}\right) .
$$

From Lemma 5.10, by using (866), we have

$$
\langle\hat{P}(n)|\hat{R}(z)| \hat{P}(m)\rangle \hat{q}(m) \hat{\eta}^{(0)}\left(z, \theta^{\prime}\right)=\left(\begin{array}{ll}
\mathcal{O}_{11} & \mathcal{O}_{12} \\
\mathcal{O}_{21} & \mathcal{O}_{22}
\end{array}\right),
$$

where

$$
\begin{aligned}
& \mathcal{O}_{11}=\mathcal{O}\left(\langle z\rangle^{-2 d(n-m)-1}\right) \hat{q}_{1}(m)+\mathcal{O}\left(\langle z\rangle^{-2 d_{12}(n-m)-2}\right) \hat{q}_{2}(m) \frac{\bar{\alpha}\left(\zeta_{-}\left(z, \theta^{\prime}\right)\right)}{\sqrt{8 z^{2}+1}}, \\
& \mathcal{O}_{12}=\mathcal{O}\left(\langle z\rangle^{-2 d(n-m)-1}\right) \hat{q}_{1}(m) \frac{\alpha\left(\zeta_{-}\left(z, \theta^{\prime}\right)\right)}{\sqrt{8 z^{2}+1}}+\mathcal{O}\left(\langle z\rangle^{-2 d_{12}(n-m)-2}\right) \hat{q}_{2}(m), \\
& \mathcal{O}_{21}=\mathcal{O}\left(\langle z\rangle^{-2 d_{21}(n-m)-2}\right) \hat{q}_{1}(m)+\mathcal{O}\left(\langle z\rangle^{-2 d(n-m)-1}\right) \hat{q}_{2}(m) \frac{\bar{\alpha}\left(\zeta_{-}\left(z, \theta^{\prime}\right)\right)}{\sqrt{8 z^{2}+1}}, \\
& \mathcal{O}_{22}=\mathcal{O}\left(\langle z\rangle^{-2 d_{21}(n-m)-2}\right) \hat{q}_{1}(m) \frac{\alpha\left(\zeta_{-}\left(z, \theta^{\prime}\right)\right)}{\sqrt{8 z^{2}+1}}+\mathcal{O}\left(\langle z\rangle^{-2 d(n-m)-1}\right) \hat{q}_{2}(m),
\end{aligned}
$$

which reads, by using (83) and (84), that

$$
\langle\hat{P}(n)|\hat{R}(z)| \hat{P}(m)\rangle \hat{q}(m) \hat{\eta}^{(0)}\left(z, \theta^{\prime}\right) \sim\left(\begin{array}{cc}
\mathcal{O}\left(N^{-1}\right) & \mathcal{O}\left(N^{-2}\right) \\
\mathcal{O}(1) & \mathcal{O}\left(N^{-1}\right)
\end{array}\right),
$$

since $d_{i j}(n) \geq 0$. Then we have

$$
\begin{aligned}
& B_{1,22}\left(z, \theta, \theta^{\prime}\right)= \frac{\bar{\alpha}\left(\zeta_{+}(z, \theta)\right)}{\sqrt{8 z^{2}+1}} \sum_{n_{2} \leq M} e^{i n \zeta_{+}(z, \theta)} \hat{q}_{1}(n) \sum_{m_{2} \leq M} e^{-i m \zeta_{-}\left(z, \theta^{\prime}\right)} \\
& \cdot\left(\langle\hat{P}(n)|\hat{R}(z)| \hat{P}(m)\rangle \hat{q}(m) \hat{\eta}^{(0)}\left(z, \theta^{\prime}\right)\right)_{12} \\
&+ \sum_{n_{2} \leq M} e^{i n \zeta_{+}(z, \theta)} \hat{q}_{2}(n) \sum_{m_{2} \leq M} e^{-i m \zeta_{-}\left(z, \theta^{\prime}\right)} \\
& \cdot\left(\langle\hat{P}(n)|\hat{R}(z)| \hat{P}(m)\rangle \hat{q}(m) \hat{\eta}^{(0)}\left(z, \theta^{\prime}\right)\right)_{22} \\
& \sim \mathcal{O}\left(N^{4 M-1}\right) .
\end{aligned}
$$

Therefore, noticing that $a_{1}(\cdot)$ in (85) is defined as (79), we can compute $\hat{q}_{2}\left(n^{(M)}\right)$ from the asymptotic expansion of $B_{22}\left(z, \theta, \theta^{\prime}\right)$.

We turn to $B_{11}\left(z, \theta, \theta^{\prime}\right)$. By using (77), (78), (81), (84), and (88), we have the following asymptotic expansion:

$$
\begin{aligned}
B_{0,11}\left(z, \theta, \theta^{\prime}\right) \sim & N^{4 M}\left(a_{1}(\theta) a_{1}\left(\theta^{\prime}\right)\right)^{M} \hat{q}_{1}\left(n^{(M)}\right) \\
& +e^{i n^{(M)}\left(\zeta_{+}(z, \theta)-\zeta_{-}\left(z, \theta^{\prime}\right)\right)} \frac{\alpha\left(\zeta_{+}(z, \theta)\right)}{\sqrt{8 z^{2}+1}} \frac{\bar{\alpha}\left(\zeta_{-}\left(z, \theta^{\prime}\right)\right)}{\sqrt{8 z^{2}+1}} \hat{q}_{2}\left(n^{(M)}\right) \\
& +\mathcal{O}\left(N^{4 M-2}\right) .
\end{aligned}
$$


Note that the asymptotic behavior of the second term of the right hand side is known, since $\hat{q}_{2}\left(n^{(M)}\right)$ has already been computed.

Let us investigate the asymptotic behavior of $B_{1,11}\left(z, \theta, \theta^{\prime}\right)$. We put

$$
\begin{aligned}
\hat{q}_{(\leq r, \leq s)}=\sum_{n_{2} \leq r} \hat{P}(n)\left(\begin{array}{cc}
\hat{q}_{1}(n) & 0 \\
0 & 0
\end{array}\right) \hat{P}(n)+\sum_{n_{2} \leq s} \hat{P}(n)\left(\begin{array}{cc}
0 & 0 \\
0 & \hat{q}_{2}(n)
\end{array}\right) \hat{P}(n), \\
\hat{q}_{(>r,>s)}=\hat{q}-\hat{q}_{(\leq r, \leq s)}, \\
\hat{H}_{(>r,>s)}=\hat{H}_{0}+\hat{q}_{(>r,>s)}, \\
\hat{R}_{(>r,>s)}(z)=\left(\hat{H}_{(>r,>s)}-z\right)^{-1} .
\end{aligned}
$$

If $r=s$, we write $\hat{q}_{\leq r}=\hat{q}_{(\leq r, \leq r)}, \hat{q}_{>r}=\hat{q}_{(>r,>r)}, \hat{H}_{>r}=\hat{H}_{(>r,>r)}$, and $\hat{R}_{>r}(z)=$ $\hat{R}_{(>r,>r)}(z)$ for brevity.

From (88), we have

$$
\left(\langle\hat{P}(n)|\hat{R}(z)| \hat{P}(m)\rangle \hat{q}(m) \hat{\eta}^{(0)}\left(z, \theta^{\prime}\right)\right)_{11} \sim \mathcal{O}\left(N^{-1}\right),
$$

which implies, by using (77) and (78), the following asymptotic expansion of the fist term of $B_{1,11}\left(z, \theta, \theta^{\prime}\right)$ :

$$
\begin{aligned}
& \sum_{n_{2} \leq M} e^{i n \zeta_{+}(z, \theta)} \hat{q}_{1}(n) \sum_{m_{2} \leq M} e^{-i m \zeta_{-}\left(z, \theta^{\prime}\right)}\left(\langle\hat{P}(n)|\hat{R}(z)| \hat{P}(m)\rangle \hat{q}(m) \hat{\eta}^{(0)}\left(z, \theta^{\prime}\right)\right)_{11} \\
& \sim \mathcal{O}\left(N^{4 M-1}\right) .
\end{aligned}
$$

We put the second term of $B_{1,11}\left(z, \theta, \theta^{\prime}\right)$ as

$I=\frac{\alpha\left(\zeta_{+}(z, \theta)\right)}{\sqrt{8 z^{2}+1}} \sum_{n \in \mathbb{Z}^{2}} e^{i n \zeta_{+}(z, \theta)} \hat{q}_{2}(n) \sum_{m \in \mathbb{Z}^{2}} e^{-i m \zeta_{-}\left(z, \theta^{\prime}\right)}\left(\langle\hat{P}(n)|\hat{R}(z)| \hat{P}(m)\rangle \hat{q}(m) \hat{\eta}^{(0)}\left(z, \theta^{\prime}\right)\right)_{21}$,

and split it into four parts: $I=I_{1}+I_{2}+I_{3}+I_{4}$, where

$$
I_{1}=\sum_{n=n^{(M)}} \sum_{m=n^{(M)}}, I_{2}=\sum_{n=n^{(M)}} \sum_{m_{2} \leq M-1}, I_{3}=\sum_{n_{2} \leq M-1} \sum_{m=n^{(M)}}, I_{4}=\sum_{n_{2} \leq M-1} \sum_{m_{2} \leq M-1} .
$$

By using (77), (78), (81), and (88), we have $I_{j} \sim \mathcal{O}\left(N^{4 M-1}\right)$ for $j=2,3$; also, $I_{4} \sim \mathcal{O}\left(N^{4 M-3}\right)$.

About $I_{1}$, by using the resolvent equation

$$
\hat{R}(z)=\hat{R}_{(>M,>M-1)}(z)-\hat{R}_{(>M,>M-1)}(z) \hat{q}_{(\leq M, \leq M-1)} \hat{R}(z),
$$

we split it into four parts: $I_{1}=I_{1,1}+I_{1,2,1}-I_{1,2,2,1}-I_{1,2,2,2}$, where

$$
\begin{aligned}
I_{1,1}= & \frac{\alpha\left(\zeta_{+}(z, \theta)\right)}{\sqrt{8 z^{2}+1}} e^{i n^{(M)}\left(\zeta_{+}(z, \theta)-\zeta_{-}\left(z, \theta^{\prime}\right)\right)} \hat{q}_{2}\left(n^{(M)}\right) \\
& \cdot\left(\left\langle\hat{P}\left(n^{(M)}\right)|\hat{R}(z)| \hat{P}\left(n^{(M)}\right)\right\rangle\left(\begin{array}{cc}
\hat{q}_{1}\left(n^{(M)}\right) & 0 \\
0 & 0
\end{array}\right) \hat{\eta}^{(0)}\left(z, \theta^{\prime}\right)\right)_{21},
\end{aligned}
$$




$$
\begin{aligned}
I_{1,2,1}= & \frac{\alpha\left(\zeta_{+}(z, \theta)\right)}{\sqrt{z^{2}+1}} e^{i n^{(M)}\left(\zeta_{+}(z, \theta)-\zeta_{-}\left(z, \theta^{\prime}\right)\right)} \hat{q}_{2}\left(n^{(M)}\right) \\
& \cdot\left(\left\langle\hat{P}\left(n^{(M)}\right)\left|\hat{R}_{(>M,>M-1)}(z)\right| \hat{P}\left(n^{(M)}\right)\right\rangle\left(\begin{array}{cc}
0 & 0 \\
0 & \hat{q}_{2}\left(n^{(M)}\right)
\end{array}\right) \hat{\eta}^{(0)}\left(z, \theta^{\prime}\right)\right)_{21}, \\
& I_{1,2,2,1}=\frac{\alpha\left(\zeta_{+}(z, \theta)\right)}{\sqrt{8 z^{2}+1}} e^{i n^{(M)}\left(\zeta_{+}(z, \theta)-\zeta_{-}\left(z, \theta^{\prime}\right)\right)} \hat{q}_{2}\left(n^{(M)}\right) \\
& \cdot\left(\left\langle\hat{P}\left(n^{(M)}\right)\left|\hat{R}_{(>M,>M-1)}(z) \hat{q}_{\leq M-1} \hat{R}(z)\right| \hat{P}\left(n^{(M)}\right)\right\rangle\right. \\
I_{1,2,2,2}= & \frac{\alpha\left(\zeta_{+}(z, \theta)\right)}{\sqrt{8 z^{2}+1}} e^{i n^{(M)}\left(\zeta_{+}(z, \theta)-\zeta_{-}\left(z, \theta^{\prime}\right)\right)} \hat{q}\left(n^{(M)}\right) \\
& \cdot\left(\left\langle\begin{array}{ll}
0 & 0 \\
0 & \hat{q}_{2}\left(n^{(M)}\right)
\end{array}\right) \hat{\eta}^{(0)}\left(z, \theta^{\prime}\right)\right)_{21}, \\
& \left.\cdot\left(\begin{array}{ll}
0 & \left.\left.\hat{P}^{(M)}\right)\left|\hat{R}_{(>M,>M-1)}(z) \hat{P}\left(n^{(M)}\right)\left(\begin{array}{cc}
\hat{q}_{1}\left(n^{(M)}\right) & 0 \\
0 & 0
\end{array}\right) \hat{P}\left(n^{(M)}\right) \hat{R}(z)\right| \hat{P}\left(n^{(M)}\right)\right\rangle \\
0 & \hat{q}_{2}\left(n^{(M)}\right)
\end{array}\right) \hat{\eta}^{(0)}\left(z, \theta^{\prime}\right)\right)_{21} \cdot
\end{aligned}
$$

We know $\hat{R}_{(>M,>M-1)}(z)$, since we have already computed $\hat{q}_{2}\left(n^{(M)}\right)$, which implies that $I_{1,2,1}$ is a known term.

By using (87) and (86), we have

$$
\left(\left\langle\hat{P}\left(n^{(M)}\right)|\hat{R}(z)| \hat{P}\left(n^{(M)}\right)\right\rangle\left(\begin{array}{cc}
\hat{q}_{1}\left(n^{(M)}\right) & 0 \\
0 & 0
\end{array}\right) \hat{\eta}^{(0)}\left(z, \theta^{\prime}\right)\right)_{21} \sim \mathcal{O}\left(N^{-2}\right),
$$

which implies, by using (77), (78), and (81), that $I_{1,1} \sim \mathcal{O}\left(N^{4 M-1}\right)$.

We can write $I_{1,2,2,1}$ as a sum of the following terms:

$$
\begin{aligned}
& \frac{\alpha\left(\zeta_{+}(z, \theta)\right)}{\sqrt{8 z^{2}+1}} e^{i n^{(M)}\left(\zeta_{+}(z, \theta)-\zeta_{-}\left(z, \theta^{\prime}\right)\right)} \\
& \cdot\left(\left\langle\hat{P}\left(n^{(M)}\right)\left|\hat{R}_{(>M,>M-1)}(z)\right| \hat{P}(k)\right\rangle \hat{q}(k)\left\langle\hat{P}(k)|\hat{R}(z)| \hat{P}\left(n^{(M)}\right)\right\rangle\right. \\
& \left.\quad \cdot\left(\begin{array}{cc}
0 & 0 \\
0 & \hat{q}\left(n^{(M)}\right)
\end{array}\right) \hat{\eta}^{(0)}\left(z, \theta^{\prime}\right)\right)_{21},
\end{aligned}
$$

where $k=\left(k_{1}, k_{2}\right) \in \mathbb{Z}^{2}, k_{2} \leq M-1$. From Lemma 5.1, we have

$$
\begin{gathered}
d\left(n^{(M)}-k\right)=d\left(k-n^{(M)}\right) \geq 1, \\
d_{21}\left(n^{(M)}-k\right)=d_{12}\left(k-n^{(M)}\right) \geq 1,
\end{gathered}
$$

since $\left(n^{(M)}-k\right)_{2}>0$ and $\left(k-n^{(M)}\right)_{2}<0$. Then, by using (73), (8), (87), (84), 
(91), and (92), we have

$$
\begin{aligned}
& \left(\left\langle\hat{P}\left(n^{(M)}\right)\left|\hat{R}_{(>M,>M-1)}(z)\right| \hat{P}(k)\right\rangle \hat{q}(k)\left\langle\hat{P}(k)|\hat{R}(z)| \hat{P}\left(n^{(M)}\right)\right\rangle\right. \\
& \left.\cdot\left(\begin{array}{cc}
0 & 0 \\
0 & \hat{q}_{2}\left(n^{(M)}\right)
\end{array}\right) \hat{\eta}^{(0)}\left(z, \theta^{\prime}\right)\right)_{21} \\
& =\mathcal{O}\left(\langle z\rangle^{-2 d_{21}\left(n^{(M)}-k\right)-2}\right) \hat{q}_{1}(k) \mathcal{O}\left(\langle z\rangle^{-2 d_{12}\left(k-n^{(M)}\right)-2}\right) \hat{q}_{2}\left(n^{(M)}\right) \frac{\bar{\alpha}\left(\zeta_{-}\left(z, \theta^{\prime}\right)\right)}{\sqrt{8 z^{2}+1}} \\
& \quad+\mathcal{O}\left(\langle z\rangle^{-2 d\left(n^{(M)}-k\right)-1}\right) \hat{q}_{2}(k) \mathcal{O}\left(\langle z\rangle^{-2 d\left(k-n^{(M)}\right)-1}\right) \hat{q}_{2}\left(n^{(M)}\right) \frac{\bar{\alpha}\left(\zeta_{-}\left(z, \theta^{\prime}\right)\right)}{\sqrt{8 z^{2}+1}} \\
& \sim \mathcal{O}\left(N^{-5}\right),
\end{aligned}
$$

which infers, by using (77), (78), and (81), that $I_{1,2,2,1} \sim \mathcal{O}\left(N^{4 M-4}\right)$.

In the same way as above, we have

$$
\begin{aligned}
& \left(\left\langle\hat{P}\left(n^{(M)}\right)\left|\hat{R}_{(>M,>M-1)}(z)\right| \hat{P}\left(n^{(M)}\right)\right\rangle\left(\begin{array}{cc}
\hat{q}_{1}\left(n^{(M)}\right) & 0 \\
0 & 0
\end{array}\right)\right. \\
& \left.\cdot\left\langle\hat{P}\left(n^{(M)}\right)|\hat{R}(z)| \hat{P}\left(n^{(M)}\right)\right\rangle\left(\begin{array}{cc}
0 & 0 \\
0 & \hat{q}_{2}\left(n^{(M)}\right)
\end{array}\right) \hat{\eta}^{(0)}\left(z, \theta^{\prime}\right)\right)_{21} \\
& =\mathcal{O}\left(\langle z\rangle^{-2 d_{21}((0,0))-2 d_{12}((0,0))-4}\right) \frac{\bar{\alpha}\left(\zeta_{-}\left(z, \theta^{\prime}\right)\right)}{\sqrt{8 z^{2}+1}} \\
& \sim \mathcal{O}\left(N^{-3}\right),
\end{aligned}
$$

which implies $I_{1,2,2,2} \sim \mathcal{O}\left(N^{-4 M-2}\right)$.

Therefore, by taking the known terms into consideration, we can compute $\hat{q}_{1}\left(n^{(M)}\right)$ from the asymptotic expansion of $B_{11}\left(z, \theta, \theta^{\prime}\right)$.

Suppose that we have computed $\hat{q}(n)$ for $n_{2} \geq p+1$. Then, by using (77), (78), (81), and (83), we have

$$
\begin{aligned}
& B_{0,22}\left(z, \theta, \theta^{\prime}\right)-\sum_{n_{2} \geq p+1} e^{i n\left(\zeta_{+}(z, \theta)-\zeta_{-}\left(z, \theta^{\prime}\right)\right)}\left(\frac{\bar{\alpha}\left(\zeta_{+}(z, \theta)\right)}{\sqrt{8 z^{2}+1}} \frac{\alpha\left(\zeta_{-}\left(z, \theta^{\prime}\right)\right)}{\sqrt{8 z^{2}+1}} \hat{q}_{1}(n)+\hat{q}_{2}(n)\right) \\
& \sim N^{4 p}\left(a_{1}(\theta) a_{1}\left(\theta^{\prime}\right)\right)^{p} \sum_{n_{2}=p}\left(b_{1}(\theta) b_{1}\left(\theta^{\prime}\right)\right)^{n_{1}} \hat{q}_{2}(n) .
\end{aligned}
$$

Let us investigate the asymptotic behavior of $B_{1,22}\left(z, \theta, \theta^{\prime}\right)=I I+I I I$, where

$$
\begin{gathered}
I I=\frac{\bar{\alpha}\left(\zeta_{+}(z, \theta)\right)}{\sqrt{8 z^{2}+1}} \sum_{n \in \mathbb{Z}^{2}} e^{i n \zeta_{+}(z, \theta)} \hat{q}_{1}(n) \\
\cdot \sum_{m \in \mathbb{Z}^{2}} e^{-i m \zeta_{-}\left(z, \theta^{\prime}\right)}\left(\langle\hat{P}(n)|\hat{R}(z)| \hat{P}(m)\rangle \hat{q}(m) \hat{\eta}^{(0)}\left(z, \theta^{\prime}\right)\right)_{12}, \\
I I I=\sum_{n \in \mathbb{Z}^{2}} e^{i n \zeta_{+}(z, \theta)} \hat{q}_{2}(n) \sum_{m \in \mathbb{Z}^{2}} e^{-i m \zeta_{-}\left(z, \theta^{\prime}\right)}\left(\langle\hat{P}(n)|\hat{R}(z)| \hat{P}(m)\rangle \hat{q}(m) \hat{\eta}^{(0)}\left(z, \theta^{\prime}\right)\right)_{22} .
\end{gathered}
$$

The following lemma is proved in the same way as above in Appendix D so is Lemma 6.2. 
Lemma 6.1. We have $I I \sim \mathcal{O}\left(N^{4 p-1}\right)$ up to a known term, which is written as

$$
\begin{aligned}
& \frac{\bar{\alpha}\left(\zeta_{+}(z, \theta)\right)}{\sqrt{8 z^{2}+1}} \sum_{n_{2} \geq p+1} e^{i n \zeta_{+}(z, \theta)} \hat{q}_{1}(n) \sum_{m_{2} \geq p+1} e^{-i m \zeta_{-}\left(z, \theta^{\prime}\right)} \\
& \cdot\left(\left\langle\hat{P}(n)\left|\hat{R}_{>p}(z)\right| \hat{P}(m)\right\rangle \hat{q}_{>p}(m) \hat{\eta}^{(0)}\left(z, \theta^{\prime}\right)\right)_{12},
\end{aligned}
$$

and $I I I \sim \mathcal{O}\left(N^{4 p-1}\right)$ up to a known term, which is

$$
\sum_{n_{2} \geq p+1} e^{i n \zeta_{+}(z, \theta)} \hat{q}_{2}(n) \sum_{m_{2} \geq p+1} e^{-i m \zeta_{-}\left(z, \theta^{\prime}\right)}\left(\left\langle\hat{P}(n)\left|\hat{R}_{>p}(z)\right| \hat{P}(m)\right\rangle \hat{q}_{>p}(m) \hat{\eta}^{(0)}\left(z, \theta^{\prime}\right)\right)_{22} .
$$

Therefore, we can compute $\hat{q}_{2}(n)$ 's for $n_{2}=p$ from (93), since the set $\left\{b_{1}(\theta) b_{1}\left(\theta^{\prime}\right) \in \mathbb{R} ; \theta, \theta^{\prime} \in(0,(1 / 2) \log 2)\right\}$ contains open intervals, where $b_{1}(\cdot)$ is defined by (80).

Finally, we compute $\hat{q}_{1}(n)$ for $n_{2}=p$. By using (77), (78), (81), and (84), we have

$$
\begin{aligned}
& B_{0,11}\left(z, \theta, \theta^{\prime}\right)-\sum_{n_{2} \geq p+1} e^{i n\left(\zeta_{+}(z, \theta)-\zeta_{-}\left(z, \theta^{\prime}\right)\right)}\left(\hat{q}_{1}(n)+\frac{\alpha\left(\zeta_{+}(z, \theta)\right)}{\sqrt{8 z^{2}+1}} \frac{\bar{\alpha}\left(\zeta_{-}\left(z, \theta^{\prime}\right)\right)}{\sqrt{8 z^{2}+1}} \hat{q}_{2}(n)\right) \\
& \sim N^{4 p}\left(a_{1}(\theta) a_{1}\left(\theta^{\prime}\right)\right)^{p} \sum_{n_{2}=p}\left(b_{1}(\theta) b_{1}\left(\theta^{\prime}\right)\right)^{n_{1}} \\
& \quad+\sum_{1}(n) \\
& \quad e_{n_{2}=p}^{i n\left(\zeta_{+}(z, \theta)-\zeta_{-}\left(z, \theta^{\prime}\right)\right)} \frac{\alpha\left(\zeta_{+}(z, \theta)\right)}{\sqrt{8 z^{2}+1}} \frac{\bar{\alpha}\left(\zeta_{-}\left(z, \theta^{\prime}\right)\right)}{\sqrt{8 z^{2}+1}} \hat{q}_{2}(n) \\
& \quad+\mathcal{O}\left(N^{4 p-2}\right) .
\end{aligned}
$$

Note that the second term of the right-hand side in (94) is known, since we have already computed $\hat{q}_{2}(n)$ 's for $n_{2}=p$.

Let us investigate the asymptotic behavior of $B_{1,11}\left(z, \theta, \theta^{\prime}\right)=I V+V$, where

$$
I V=\sum_{n \in \mathbb{Z}^{2}} e^{i n \zeta_{+}(z, \theta)} \hat{q}_{1}(n) \sum_{m \in \mathbb{Z}^{2}} e^{-i m \zeta_{-}\left(z, \theta^{\prime}\right)}\left(\langle\hat{P}(n)|\hat{R}(z)| \hat{P}(m)\rangle \hat{q}(m) \hat{\eta}^{(0)}\left(z, \theta^{\prime}\right)\right)_{11},
$$

and $V=I$. By using the next lemma, we can compute $\hat{q}_{1}(n)$ 's for $n_{2}=p$ from (94) as before.

Lemma 6.2. We have $I V \sim \mathcal{O}\left(N^{4 p-1}\right)$ up to a known term, which is written as

$$
\begin{aligned}
& \sum_{n_{2} \geq p+1} e^{i n \zeta_{+}(z, \theta)} \hat{q}_{1}(n) \sum_{m_{2} \geq p+1} e^{-i m \zeta_{-}\left(z, \theta^{\prime}\right)} \\
& \cdot\left(\left\langle\hat{P}(n)\left|\hat{R}_{(>p,>p-1)}(z)\right| \hat{P}(m)\right\rangle \hat{q}_{(>p,>p-1)}(m) \hat{\eta}^{(0)}\left(z, \theta^{\prime}\right)\right)_{11},
\end{aligned}
$$

and $V \sim \mathcal{O}\left(N^{4 p-1}\right)$ up to a known term, which is

$$
\begin{aligned}
& \frac{\alpha\left(\zeta_{+}(z, \theta)\right)}{\sqrt{8 z^{2}+1}} \sum_{n_{2} \geq p} e^{i n \zeta_{+}(z, \theta)} \hat{q}_{2}(n) \sum_{m_{2} \geq p+1} e^{-i m \zeta_{-}\left(z, \theta^{\prime}\right)} \\
& \cdot\left(\left\langle\hat{P}(n)\left|\hat{R}_{(>p,>p-1)}(z)\right| \hat{P}(m)\right\rangle \hat{q}_{(>p,>p-1)}(m) \hat{\eta}^{(0)}\left(z, \theta^{\prime}\right)\right)_{21} .
\end{aligned}
$$


Thus, we can compute all the $q(n)$ 's inductively.

\section{The triangle lattice}

Let $G$ be the triangle lattice. Then, in the same way as in Section 2, we have $\hat{H}_{0} \simeq 6\left(\Delta_{d}+1\right)$ on $l^{2}\left(\mathbb{Z}^{2}\right) \simeq l^{2}(G)$ as follows:

$$
\begin{aligned}
\left(\hat{H}_{0} \hat{f}\right)(n)= & \hat{f}\left(n_{1}+1, n_{2}\right)+\hat{f}\left(n_{1}-1, n_{2}\right)+\hat{f}\left(n_{1}, n_{2}+1\right)+\hat{f}\left(n_{1}, n_{2}-1\right) \\
& +\hat{f}\left(n_{1}-1, n_{2}+1\right)+\hat{f}\left(n_{1}+1, n_{2}-1\right)
\end{aligned}
$$

for $\hat{f}=(\hat{f}(n))_{n \in \mathbb{Z}^{2}}$. See Figure 6 .

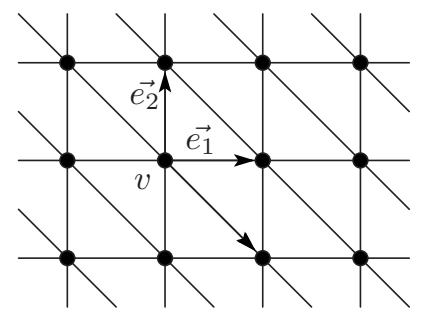

Figure 6: the triangle lattice

In this case, the Fourier transform $\mathcal{F}$ is just the Fourier series on $\mathbb{Z}^{2}$, which implies that $H_{0}=\mathcal{F} \hat{H}_{0} \mathcal{F}^{*}$ is a multiplication operator by

$$
p(\xi)=2\left(\cos \xi_{1}+\cos \xi_{2}+\cos \left(\xi_{1}-\xi_{2}\right)\right)
$$

on $L^{2}\left(\mathbb{T}^{2}\right)$. Here, we note the similarity between (95) and the square of (5). Therefore, Mourre estimate is obtained with the conjugate operator $A=\nabla p$. $\nabla+\nabla \cdot \nabla p$ on any closed interval $I \subset(-3,6) \backslash\{0\}$. Note also that we no longer need cut-off functions which appear in the hexagonal lattice case. By using limiting absorption principle, we have a spectral representation for $\hat{H}=\hat{H}_{0}+\hat{q}$; also, a representation for the kernel of the scattering amplitude $A\left(\lambda ; \theta, \theta^{\prime}\right)$, where $\theta$ and $\theta^{\prime} \in \mathbb{R}$ are local coordinates of $\mathcal{M}_{\lambda}=\left\{\xi \in \mathbb{T}^{2} ; p(\xi)=\lambda\right\}$, which satisfy

$$
2\left(\cos \xi_{1}(k, \theta)+\cos \xi_{2}(k, \theta)+\cos \left(\xi_{1}(k, \theta)-\xi_{2}(k, \theta)\right)\right)= \pm \sqrt{k} .
$$

We have the analytic continuations $\zeta_{ \pm}(z, \theta)=\left(\zeta_{ \pm, 1}(z, \theta), \zeta_{ \pm, 2}(z, \theta)\right)$ from $\xi(\lambda, \theta)=\left(\xi_{1}(\lambda, \theta), \xi_{2}(\lambda, \theta)\right)$ in the same way as in Section 4; also, the resolvent estimates as in Isozaki and Korotyaev [9, where we only have to replace $|\cdot|_{l^{1}}$

with $d(\cdot)$. Therefore, we can adopt the same strategy for our reconstruction procedure for the potential as in the hexagonal lattice. 


\section{Acknowledgment}

I would like to thank Professors Hiroshi Isozaki and Evgeny Korotyaev for their encouragements and invaluable suggestions about the scattering and the inverse scattering theories for discrete Schrödinger operators. I also thank Mr. Hisashi Morioka for helpful discussions.

\section{Appendix A}

\section{A.1 Proof of Theorem 3.1}

From Lemma 3.6. it is uniquely extended to

$$
\mathcal{F}_{0}: L^{2}\left(\mathbb{T}^{2} ; \mathbb{C}^{2}\right) \rightarrow L^{2}\left((-3,3) ; L^{2}\left(\mathcal{M}_{|\lambda|} ; \mathbb{C}^{2}\right) ; d \lambda\right)
$$

as an isometry. To prove that $\mathcal{F}_{0}$ is onto, we note that $\mathcal{F}_{0}$ maps $\cup_{\varepsilon}>0 C_{\varepsilon}^{\infty}\left(\mathbb{T}^{2} ; \mathbb{C}^{2}\right)$ to a dense set in $L^{2}\left((-3,3) ; L^{2}\left(\mathcal{M}_{|\lambda|} ; \mathbb{C}^{2}\right) ; d \lambda\right)$.

The second statement is already proved in (18).

We note that

$$
\begin{aligned}
\left(\int_{I_{N}} \mathcal{F}_{0}(\lambda)^{*} g(\lambda) d \lambda, f\right)_{L^{2}\left(\mathbb{T}^{2} ; \mathbb{C}^{2}\right)} & =\int_{I_{N}}\left(\mathcal{F}_{0}(\lambda)^{*} g(\lambda), f\right)_{L^{2}\left(\mathbb{T}^{2} ; \mathbb{C}^{2}\right)} d \lambda \\
& =\int_{I_{N}}\left(g(\lambda), \mathcal{F}_{0}(\lambda) f\right)_{L^{2}\left(\mathcal{M}_{|\lambda|} ; \mathbb{C}^{2}\right)} d \lambda
\end{aligned}
$$

for $f \in \mathcal{H}^{s}, s>1 / 2$, and $g \in L^{2}\left((-3,3) ; L^{2}\left(\mathcal{M}_{|\lambda|} ; \mathbb{C}^{2}\right) ; d \lambda\right)$ (Reed-Simon [22]), which implies that

$$
\left.\mid\left(\int_{I_{N}} \mathcal{F}_{0}(\lambda)^{*} g(\lambda) d \lambda, f\right)_{L^{2}\left(\mathbb{T}^{2} ; \mathbb{C}^{2}\right)}\right) \mid \leq\|g\|_{L^{2}\left((-3,3) ; L^{2}\left(\mathcal{M}_{|\lambda|} ; \mathbb{C}^{2}\right) ; d \lambda\right)}\|f\|_{L^{2}\left(\mathbb{T}^{2} ; \mathbb{C}^{2}\right)} .
$$

Then, by Riesz's theorem, we have

$$
\begin{gathered}
\int_{I_{N}} \mathcal{F}_{0}(\lambda)^{*} g(\lambda) d \lambda \in L^{2}\left(\mathbb{T}^{2} ; \mathbb{C}^{2}\right), \\
\left\|\int_{I_{N}} \mathcal{F}_{0}(\lambda)^{*} g(\lambda) d \lambda\right\| \leq\|g\|_{L^{2}\left((-3,3) ; L^{2}\left(\mathcal{M}_{|\lambda|} ; \mathbb{C}^{2}\right) ; d \lambda\right) .}
\end{gathered}
$$

Let $I_{N}, N=1,2, \cdots$, be a finite union of compact intervals as such in the third statement. Then we have

$$
\begin{aligned}
& \left\|\int_{I_{N}} \mathcal{F}_{0}(\lambda)^{*}\left(\mathcal{F}_{0} f\right)(\lambda) d \lambda-\int_{I_{M}} \mathcal{F}_{0}(\lambda)^{*}\left(\mathcal{F}_{0} f\right)(\lambda) d \lambda\right\|_{L^{2}\left(\mathbb{T}^{2} ; \mathbb{C}^{2}\right)}^{2} \\
& =\left\|\int_{I_{N} \Delta I_{M}} \mathcal{F}_{0}(\lambda)^{*}\left(\mathcal{F}_{0} f\right)(\lambda) d \lambda\right\|_{L^{2}\left(\mathbb{T}^{2} ; \mathbb{C}^{2}\right)}^{2} \\
& \leq\left\|\chi_{I_{N} \Delta I_{M}} \mathcal{F}_{0} f\right\|_{L^{2}\left((-3,3) ; L^{2}\left(\mathcal{M}_{|\lambda|} ; \mathbb{C}^{2}\right) ; d \lambda\right)}^{2} \\
& =\int_{I_{N} \Delta I_{M}}\left\|\mathcal{F}_{0} f\right\|_{L^{2}\left(\mathcal{M}_{|\lambda|} ; \mathbb{C}^{2}\right)} d \lambda \\
& \rightarrow 0
\end{aligned}
$$


as $M, N \rightarrow \infty$, since $\mathcal{F}_{0} f \in L^{2}\left((-3,3) ; L^{2}\left(\mathcal{M}_{|\lambda|} ; \mathbb{C}^{2} ; d \lambda\right)\right)$, which implies that $\int_{I_{N}} \mathcal{F}_{0}(\lambda)^{*}\left(\mathcal{F}_{0} f\right)(\lambda) d \lambda, N=1,2, \cdots$, is Cauchy.

Therefore, there exists $f_{0} \in L^{2}\left(\mathbb{T}^{2} ; \mathbb{C}^{2}\right)$ such that

$$
f_{0}=\mathrm{s}-\lim _{N \rightarrow \infty} \int_{I_{N}} \mathcal{F}_{0}(\lambda)^{*}\left(\mathcal{F}_{0} f\right)(\lambda) d \lambda .
$$

By Parseval's formula, we have $f=f_{0}$, since

$$
\begin{aligned}
\left(f_{0}, h\right)_{L^{2}\left(\mathbb{T}^{2} ; \mathbb{C}^{2}\right)} & =\lim _{N \rightarrow \infty} \int_{I_{N}}\left(\mathcal{F}_{0}(\lambda)^{*}\left(\mathcal{F}_{0} f\right)(\lambda), h\right)_{L^{2}\left(\mathcal{M}_{|\lambda|} ; \mathbb{C}^{2}\right)} d \lambda \\
& =\lim _{N \rightarrow \infty} \int_{I_{N}}\left(\mathcal{F}_{0}(\lambda) f, \mathcal{F}_{0}(\lambda) h\right)_{L^{2}\left(\mathcal{M}_{|\lambda|} ; \mathbb{C}^{2}\right)} d \lambda \\
& =\left(\mathcal{F}_{0} f, \mathcal{F}_{0} h\right)_{L^{2}\left((-3,3) ; L^{2}\left(\mathcal{M}_{|\lambda|} ; \mathbb{C}^{2}\right) ; d \lambda\right)} \\
& =(f, h)_{L^{2}\left(\mathbb{T}^{2} ; \mathbb{C}^{2}\right)}
\end{aligned}
$$

for any $h \in C^{\infty}\left(\mathbb{T}^{2} ; \mathbb{C}^{2}\right)$.

\section{A.2 Proof of Theorem 3.2}

By the limiting absorption principle and the virial theorem, the eigenvalues of $H$ in $(-3,3) \backslash\{ \pm 1,0\}$ accumulate, if possible, only at $\pm 3, \pm 1$ or 0 and there is no singularly continuous spectrum.

Let $-3<a<b<3$. Assume that the closed interval $[a, b]$ does not contain the eigenvalues of $H$. Then, by Stone's theorem, we have

$$
\begin{aligned}
& \frac{1}{2 \pi} \int_{a}^{b}((R(\lambda+i 0)-R(\lambda-i 0)) f, g)_{L^{2}\left(\mathbb{T}^{2} ; \mathbb{C}^{2}\right)} \\
= & \left(\left(E_{H_{a c}}((-\infty, b])-E_{H_{a c}}((-\infty, a])\right) f, g\right)_{L^{2}\left(\mathbb{T}^{2} ; \mathbb{C}^{2}\right)},
\end{aligned}
$$

which implies that $\mathcal{F}^{( \pm)}$are partial isometries in the same way as Theorem 3.1

From (19), $\mathcal{F}^{( \pm)}$diagonalize $H$, which proves the first statement. have

Let $f^{\perp} \in \mathcal{H}_{a c}(H)^{\perp}$. Then, for any $g \in L^{2}\left((-3,3) ; L^{2}\left(\mathcal{M}_{|\lambda|} ; \mathbb{C}^{2}\right) ; d \lambda\right)$, we

$$
\begin{aligned}
\left(\int_{I_{N}} \mathcal{F}^{( \pm)}(\lambda)^{*} g(\lambda), f^{\perp}\right)_{L^{2}\left(\mathbb{T}^{2} ; \mathbb{C}^{2}\right)} & =\int_{I_{N}}\left(\mathcal{F}^{( \pm)}(\lambda)^{*} g(\lambda), f^{\perp}\right)_{L^{2}\left(\mathbb{T}^{2} ; \mathbb{C}^{2}\right)} d \lambda \\
& =\int_{I_{N}}\left(g(\lambda), \mathcal{F}^{( \pm)}(\lambda) f^{\perp}\right)_{L^{2}\left(\mathcal{M}_{|\lambda|} ; \mathbb{C}^{2}\right)} d \lambda \\
& =\left(g, \mathcal{F}^{( \pm)} f^{\perp}\right)_{L^{2}\left((-3,3) ; L^{2}\left(\mathcal{M}_{|\lambda|} ; \mathbb{C}^{2}\right) ; d \lambda\right)} \\
& =0,
\end{aligned}
$$

since $\mathcal{F}^{( \pm)} f^{\perp}=0$, which implies, By Riesz's theorem, that

$$
\int_{I_{N}} \mathcal{F}^{( \pm)}(\lambda)^{*} g(\lambda) d \lambda \in \mathcal{H}_{a c}(H) .
$$

Thus we can prove the second statement in the same way as in Theorem 3.1 To prove the third statement, we only have to take the adjoint of (19). 


\section{Appendix B}

\section{B.1 Proof of (63) and (64)}

Let $\cos \frac{E_{1}}{2}=x_{c, 1}+i y_{c, 1}$. Then, from (51), we have

$$
\begin{aligned}
& x_{c, 1}=c(\theta)\left\{1-2 \frac{\sinh ^{2} \theta}{\left(2-e^{2 \theta}\right)^{2}} N^{-2}+\mathcal{O}\left(N^{-4}\right)\right\}, \\
& y_{c, 1}=c(\theta)\left\{-4 \frac{\sinh ^{2} \theta}{\left(2-e^{2 \theta}\right)^{2}} N^{-3}+\mathcal{O}\left(N^{-5}\right)\right\},
\end{aligned}
$$

where

$$
c=c(\theta)=\frac{e^{-\theta}}{\sqrt{2-e^{2 \theta}}} .
$$

Also, we have

$$
\begin{aligned}
& \cos \frac{\eta_{1}}{2} \cosh \frac{\kappa_{1}}{2}=x_{c, 1}, \\
& -\sin \frac{\eta_{1}}{2} \sinh \frac{\kappa_{1}}{2}=y_{c, 1},
\end{aligned}
$$

where $E_{1}=\eta_{1}+i \kappa_{1}$. From (97), we have $y_{c, 1}<0$ for sufficiently large $N>0$, which implies that $\sinh \frac{\kappa_{1}}{2}>0$, since we have already proved that $\sin \frac{\eta_{1}}{2}>0$ in Section 4.

From (98) and (99), we have

$$
\left(1-\sin ^{2} \frac{\eta_{1}}{2}\right)\left(\sin ^{2} \frac{\eta_{1}}{2}+y_{c, 1}^{2}\right)=x_{c, 1}^{2} \sin ^{2} \frac{\eta_{1}}{2} .
$$

We put

$$
t_{1}=\sin ^{2} \frac{\eta_{1}}{2}
$$

Then, from (100), we have

$$
t_{1}^{2}+\left(x_{c, 1}{ }^{2}+y_{c, 1}{ }^{2}-1\right) t_{1}-y_{c, 1}{ }^{2}=0 .
$$

By solving (102), we have

$$
t_{1}=\frac{1}{2}\left\{-\left(x_{c, 1}^{2}+y_{c, 1}^{2}-1\right)+\sqrt{\left(x_{c, 1}^{2}+y_{c, 1}^{2}-1\right)^{2}+4 y_{c, 1}^{2}}\right\},
$$

since $t_{1}>0$. By using (96) and (97), we can calculate

$$
x_{c, 1}^{2}+y_{c, 1}^{2}-1=c^{2}-1-4 c^{2} \frac{\sinh ^{2} \theta}{\left(2-e^{2 \theta}\right)^{2}} N^{-2}+\mathcal{O}\left(N^{-4}\right),
$$

which implies

$$
x_{c, 1}^{2}+y_{c, 1}^{2}-1>0
$$


for sufficiently large $N>0$, since

$$
c^{2}-1=4 \frac{\sinh ^{2} \theta}{2-e^{2 \theta}}>0 .
$$

Noticing (104) and

$$
y_{c, 1}{ }^{2}\left(x_{c, 1}{ }^{2}+y_{c, 1}{ }^{2}-1\right)^{-2}=\mathcal{O}\left(N^{-6}\right),
$$

we have, from (103),

$$
\begin{aligned}
t_{1}= & \frac{1}{2}\left\{-\left(x_{c, 1}{ }^{2}+y_{c, 1}{ }^{2}-1\right)\right. \\
& \left.\quad+\left(x_{c, 1}{ }^{2}+y_{c, 1}{ }^{2}-1\right)\left(1+2 y_{c, 1}{ }^{2}\left(x_{c, 1}{ }^{2}+y_{c, 1}{ }^{2}-1\right)^{-2}+\mathcal{O}\left(N^{-12}\right)\right)\right\} \\
= & y_{c, 1}{ }^{2}\left(x_{c, 1}{ }^{2}+y_{c, 1}{ }^{2}-1\right)+\mathcal{O}\left(N^{-12}\right) .
\end{aligned}
$$

Since $\sin \frac{\eta_{1}}{2}>0$ and $y_{c, 1}<0$ for sufficiently large $N>0$, we have, from (101) and (105),

$$
\begin{aligned}
\sin \frac{\eta_{1}}{2} & =-y_{c, 1}\left(x_{c, 1}{ }^{2}+y_{c, 1}^{2}-1\right)^{-1 / 2}+\mathcal{O}\left(N^{-9}\right) \\
& =2 \frac{c}{\sqrt{c^{2}-1}} \frac{\sinh \theta}{\left(2-e^{2 \theta}\right)^{2}} N^{-3}+\mathcal{O}\left(N^{-5}\right) \\
& =\frac{e^{-\theta}}{\left(2-e^{2 \theta}\right)^{2}} N^{-3}+\mathcal{O}\left(N^{-5}\right) .
\end{aligned}
$$

Therefore, we have

$$
\eta_{1}=2 m_{1} \pi+\mathcal{O}\left(N^{-3}\right)
$$

for some $m_{1} \in \mathbb{Z}$.

From (97) and (99), we can calculate

$$
\begin{aligned}
\sinh \frac{\kappa_{1}}{2} & =-\frac{y_{c, 1}}{\sin \frac{\eta_{1}}{2}} \\
& =\left(x_{c, 1}{ }^{2}+y_{c, 1}{ }^{2}-1\right)^{-1 / 2}+\mathcal{O}\left(N^{-6}\right) \\
& =\sqrt{c^{2}-1}+\mathcal{O}\left(N^{-2}\right) \\
& =b+\mathcal{O}\left(N^{-2}\right),
\end{aligned}
$$

where $b=b(\theta)$ is defined by (40). We put

$$
s_{1}=\sinh \frac{\kappa_{1}}{2} .
$$

From (106), we note that $s_{1} \rightarrow b>0$ as $N \rightarrow+\infty$. Then we have

$$
\begin{aligned}
e^{\kappa_{1} / 2} & =s_{1}+\sqrt{s_{1}^{2}+1} \\
& =b+\sqrt{b^{2}+1}+\mathcal{O}\left(N^{-2}\right),
\end{aligned}
$$

which implies that

$$
\frac{\kappa_{1}}{2}=\log \left(b+\sqrt{b^{2}+1}\right)+\mathcal{O}\left(N^{-2}\right) .
$$




\section{B.2 Proof of (65) and (66)}

Let $\sin \frac{E_{2}}{2}=x_{2}+i y_{2}$. Then, from (501), we have

$$
\begin{gathered}
x_{s, 2}=a(\theta)\left\{-N-\frac{1}{2} \frac{e^{-2 \theta}}{\left(2-e^{2 \theta}\right)^{2}} N^{-1}+\mathcal{O}\left(N^{-3}\right)\right\}, \\
y_{s, 2}=a(\theta)\left\{1-\frac{1}{2} \frac{e^{-2 \theta}}{\left(2-e^{2 \theta}\right)^{2}} N^{-2}+\mathcal{O}\left(N^{-4}\right)\right\},
\end{gathered}
$$

where $a=a(\theta)$ is defined by (39). Also, we have

$$
\begin{aligned}
& \sin \frac{\eta_{2}}{2} \cosh \frac{\kappa_{2}}{2}=x_{s, 2}, \\
& \cos \frac{\eta_{2}}{2} \sinh \frac{\kappa_{2}}{2}=y_{s, 2},
\end{aligned}
$$

where $E_{2}=\eta_{2}+i \kappa_{2}$. From (108), we have $y_{s, 2}>0$ for sufficiently large $N>0$, which implies that $\sinh \frac{\kappa_{2}}{2}>0$, since we have already proved that $\cos \frac{\eta_{2}}{2}>0$ in Section 4

From (109) and (110), we have

$$
\left(1-\cos ^{2} \frac{\eta_{2}}{2}\right)\left(\cos ^{2} \frac{\eta_{2}}{2}+y_{s, 2}^{2}\right)=x_{s, 2}^{2} \cos ^{2} \frac{\eta_{2}}{2} .
$$

We put

$$
t_{2}=\cos ^{2} \frac{\eta_{2}}{2}
$$

Then, from (111), we have

$$
t_{2}^{2}+\left(x_{s, 2}^{2}+y_{s, 2}^{2}-1\right) t_{2}-y_{s, 2}^{2}=0 .
$$

By solving (113), we have

$$
t_{2}=\frac{1}{2}\left\{-\left(x_{s, 2}^{2}+y_{s, 2}^{2}-1\right)+\sqrt{\left(x_{s, 2}^{2}+y_{s, 2}^{2}-1\right)^{2}+4 y_{s, 2}^{2}}\right\},
$$

since $t_{2}>0$. By using (107) and (108), we can calculate

$$
\begin{aligned}
x_{s, 2}{ }^{2}+y_{s, 2}{ }^{2}-1 & =\left(2-e^{2 \theta}\right) N^{2}+\frac{e^{-2 \theta}}{2-e^{2 \theta}}+1-e^{2 \theta}+\mathcal{O}\left(N^{-2}\right) \\
& =\mathcal{O}\left(N^{2}\right) .
\end{aligned}
$$

Noticing that $2-e^{2 \theta}>0$ for $0<\theta<\frac{1}{2} \log 2$, we have

$$
\begin{gathered}
x_{s, 2}^{2}+y_{s, 2}^{2}-1>0, \\
y_{s, 2}^{2}\left(x_{s, 2}^{2}+y_{s, 2}^{2}-1\right)^{-2}=\mathcal{O}\left(N^{-4}\right),
\end{gathered}
$$


for sufficiently large $N>0$. Therefore, by using (115) and (116), we have, from (114),

$$
\begin{aligned}
t_{2}= & \frac{1}{2}\left\{-\left(x_{s, 2}^{2}+y_{s, 2}^{2}-1\right)\right. \\
& \left.\quad+\left(x_{s, 2}{ }^{2}+y_{s, 2}^{2}-1\right)\left(1+2 y_{s, 2}^{2}\left(x_{s, 2}^{2}+y_{s, 2}^{2}-1\right)^{-2}+\mathcal{O}\left(N^{-8}\right)\right)\right\} \\
= & y_{s, 2}{ }^{2}\left(x_{s, 2}{ }^{2}+y_{s, 2}{ }^{2}-1\right)^{-1}+\mathcal{O}\left(N^{-6}\right) \\
= & N^{-2}+\mathcal{O}\left(N^{-4}\right) .
\end{aligned}
$$

Since $\cos \frac{\eta_{2}}{2}>0$, we have, from (112) and (117),

$$
\cos \frac{\eta_{2}}{2}=N^{-1}+\mathcal{O}\left(N^{-3}\right) .
$$

Thus, we have

$$
\eta_{2}=\left(2 m_{2}+1\right) \pi+\mathcal{O}\left(N^{-1}\right)
$$

for some $m_{2} \in \mathbb{Z}$.

From (108) and (110), we can calculate

$$
\begin{aligned}
\sinh \frac{\kappa_{2}}{2} & =\frac{y_{s, 2}}{\cos \frac{\eta_{2}}{2}} \\
& =a N+\mathcal{O}\left(N^{-1}\right) .
\end{aligned}
$$

We put

$$
s_{2}=\sinh \frac{\kappa_{2}}{2} \text {. }
$$

From (118), we note that $s_{2} \rightarrow+\infty$ as $N \rightarrow+\infty$. Then we have

$$
\begin{aligned}
e^{\kappa_{2} / 2} & =s_{2}+\sqrt{s_{2}^{2}+1} \\
& =2 s_{2}+\frac{1}{2} s_{2}{ }^{-1}+\mathcal{O}\left(s_{2}{ }^{-3}\right) \\
& =2 a N+\mathcal{O}\left(N^{-1}\right),
\end{aligned}
$$

which implies that

$$
\frac{\kappa_{2}}{2}=\log (2 a N)+\mathcal{O}\left(N^{-2}\right)
$$

\section{Appendix C}

\section{C.1 The proof of Lemma 5.4}

If $i=j$, these inequalities are reduced to the triangle inequality (69).

Assume that $(i, j)=(1,2)$. We prove the inequality (70). For $m=\left(m_{1}, m_{2}\right)$, $n=\left(n_{1}, n_{2}\right) \in \mathbb{Z}^{2}$, we treat the following four cases:

(i) $m_{1}-n_{1}>0, m_{2}-n_{2}>0$, 
(ii) $m_{1}-n_{1} \leq 0, m_{2}-n_{2}>0$,

(iii) $m_{1}-n_{1} \leq 0, m_{2}-n_{2} \leq 0$,

(iv) $m_{1}-n_{1}>0, m_{2}-n_{2} \leq 0$.

Let us consider the case (iii). By taking the location of $l=\left(l_{1}, l_{2}\right) \in \mathbb{Z}^{2}$ into consideration, we treat the following seven cases:

(iii.1) $\left(m_{1}-l_{1}\right)\left(m_{2}-l_{2}\right) \geq 0, l_{1}-n_{1} \leq 0, l_{2}-n_{2}>0$,

(iil.2) $\left(m_{1}-l_{1}\right)\left(m_{2}-l_{2}\right) \leq 0, l_{1}-n_{1} \leq 0, l_{2}-n_{2}>0$,

(iii.3) $m_{1}-l_{1} \leq 0, m_{2}-l_{2} \geq 0, l_{1}-n_{1} \leq 0, l_{2}-n_{2} \leq 0$,

(iii.4) $m_{1}-l_{1} \geq 0, m_{2}-l_{2} \geq 0, l_{1}-n_{1} \leq 0, l_{2}-n_{2} \leq 0$,

(iii.5) $m_{1}-l_{1} \leq 0, m_{2}-l_{2} \geq 0, l_{1}-n_{1}>0, l_{2}-n_{2}>0$,

(iil.6) $m_{1}-l_{1} \leq 0, m_{2}-l_{2} \leq 0, l_{1}-n_{1}>0, l_{2}-n_{2}>0$,

(iii.7) $m_{1}-l_{1} \leq 0, m_{2}-l_{2} \geq 0, l_{1}-n_{1} \leq 0, l_{2}-n_{2} \leq 0$.

Let us consider, for example, the case (ii 5). Then we have

$$
\begin{aligned}
d_{12}(m-n) & =\max \left\{\left|m_{1}-n_{1}\right|,\left|m_{2}-n_{2}\right|-1\right\} \\
& \leq \max \left\{\left|m_{1}-l_{1}\right|,\left|m_{2}-l_{2}\right|\right\}+\max \left\{\left|l_{1}-n_{1}\right|,\left|l_{2}-n_{2}\right|-1\right\} .
\end{aligned}
$$

By the assumption, we have $\left|l_{2}-n_{2}\right|-1 \geq 0$, which implies

$$
\begin{aligned}
d_{12}(m-n) & \leq \max \left\{\left|m_{1}-l_{1}\right|,\left|m_{2}-l_{2}\right|\right\}+\left|l_{1}-n_{1}\right|+\left|l_{2}-n_{2}\right|-1 \\
& =d_{11}(m-l)+d_{12}(l-n) .
\end{aligned}
$$

We can treat the other cases of (iii) similarly; also, the cases (ii), (iii), and (iv). We can also prove (70) for $(i, j)=(2,1)$ by the similar arguments.

By using (70), we have

$$
\begin{aligned}
d_{i j}(m-n) & =d_{j i}(n-m) \\
& \leq d_{j j}(n-l)+d_{j i}(l-m) \\
& =d_{i j}(m-l)+d_{j j}(l-n),
\end{aligned}
$$

which proves the inequality (71).

\section{C.2 The proof of Lemma 5.5}

If $i=j$, these inequalities are reduced to the triangle inequality (69).

Assume $(i, j)=(1,2)$. For $m=\left(m_{1}, m_{2}\right), n=\left(n_{1}, n_{2}\right) \in \mathbb{Z}^{2}$, we treat the following eight cases:

(i) $m_{1}-n_{1}>0, m_{2}-n_{2}>0$, 
(ii) $m_{1}-n_{1}<0, m_{2}-n_{2}>0$,

(iii) $m_{1}-n_{1}<0, m_{2}-n_{2}<0$,

(iv) $m_{1}-n_{1}>0, m_{2}-n_{2}<0$,

(v) $m_{1}=n_{1}, m_{2}-n_{2}>0$,

(vi) $m_{1}=n_{1}, m_{2}-n_{2}<0$,

(vii) $m_{1}-n_{1}>0, m_{2}=n_{2}$,

(viii) $m_{1}-n_{1}<0, m_{2}=n_{2}$.

Let us consider the case (iii). We treat the following nine cases, depending on the location of $l=\left(l_{1}, l_{2}\right) \in \mathbb{Z}^{2}$ :

(iii) 1$) l_{1}-m_{1}<0, l_{2}-n_{2} \geq 0, m_{1}-l_{1} \leq 0, m_{2}-l_{2}>0$,

(iii 2) $l_{1}-m_{1}<0, l_{2}-n_{2} \geq 0, m_{1}-l_{1} \leq 0, m_{2}-l_{2} \leq 0$,

(iii.3) $l_{1}-m_{1}<0, l_{2}-n_{2} \geq 0, m_{1}-l_{1}>0, m_{2}-l_{2}>0$,

(iii)4) $l_{1}-m_{1}<0, l_{2}-n_{2} \geq 0, m_{1}-l_{1}>0, m_{2}-l_{2} \leq 0$,

(iii.5) $l_{1}-m_{1} \geq 0, l_{2}-n_{2} \geq 0, m_{1}-l_{1} \leq 0, m_{2}-l_{2}>0$,

(iii.6) $l_{1}-m_{1} \geq 0, l_{2}-n_{2} \geq 0, m_{1}-l_{1} \leq 0, m_{2}-l_{2} \leq 0$,

(iii.7) $l_{1}-m_{1}<0, l_{2}-n_{2} \geq 0, m_{1}-l_{1} \leq 0, m_{2}-l_{2}>0$,

(iii.8) $l_{1}-m_{1}<0, l_{2}-n_{2}<0, m_{1}-l_{1}>0, m_{2}-l_{2}>0$,

(iii.9) $l_{1}-m_{1} \geq 0, l_{2}-n_{2}<0, m_{1}-l_{1} \leq 0, m_{2}-l_{2}>0$.

Let us consider, for example, the case (iii3). Then we have

$$
\begin{aligned}
d_{11}(m-n) & =\max \left\{\left|m_{1}-n_{1}\right|,\left|m_{2}-n_{2}\right|\right\} \\
& =\max \left\{\left(n_{1}-l_{1}\right)+\left(l_{1}-m_{1}\right),\left|m_{2}-n_{2}\right|\right\} .
\end{aligned}
$$

By the assumption, we have $l_{1}-m_{1}<0$ and $\left|m_{2}-l_{2}\right|-1 \geq 0$, which implies that

$$
\begin{aligned}
d_{11}(m-n) & \leq \max \left\{\left|n_{1}-l_{1}\right|,\left|m_{2}-n_{2}\right|\right\} \\
& \leq \max \left\{\left|l_{1}-n_{1}\right|-1,\left|m_{2}-l_{2}\right|+\left|l_{2}-n_{2}\right|-1\right\}+1 \\
& \leq\left(\left|m_{2}-l_{2}\right|-1\right)+\max \left\{\left|l_{1}-n_{1}\right|-1,\left|l_{2}-n_{2}\right|\right\}+1 \\
& \leq d_{12}(m-l)+d_{21}(l-n)+1 .
\end{aligned}
$$
(iv).

We can treat the other cases of (iii) similarly; also, the cases (ii), (iii), and About (vil), we treat the following six cases: 
vi.1) $l_{1}-n_{1} \geq 0, l_{2}-n_{2}<0, m_{1}-l_{1} \leq 0, m_{2}-l_{2}>0$,

(vi)2) $l_{1}-n_{1} \geq 0, l_{2}-n_{2}<0, m_{1}-l_{1} \leq 0, m_{2}-l_{2} \leq 0$,

(vil.3) $l_{1}-n_{1}<0, l_{2}-n_{2}<0, m_{1}-l_{1}>0, m_{2}-l_{2}>0$,

vi 4) $l_{1}-n_{1}<0, l_{2}-n_{2}<0, m_{1}-l_{1}>0, m_{2}-l_{2} \leq 0$,

vi.5) $l_{1}-n_{1} \geq 0, l_{2}-n_{2} \geq 0, m_{1}-l_{1} \leq 0, m_{2}-l_{2} \leq 0$,

vil6) $l_{1}-n_{1}<0, l_{2}-n_{2} \geq 0, m_{1}-l_{1}>0, m_{2}-l_{2} \leq 0$.

Let us consider, for example, the case (vil1). Then we have

$$
\begin{aligned}
d_{11}(m-n) & =\left|m_{2}-n_{2}\right| \\
& =\left(n_{2}-l_{2}\right)+\left(l_{2}-m_{2}\right) .
\end{aligned}
$$

By the assumption, we have $l_{2}-m_{2}<0$, which implies

$$
\begin{aligned}
d_{11}(m-n) & \leq\left(\left|l_{2}-n_{2}\right|-1\right)+1 \\
& \leq \max \left\{\left|l_{1}-n_{1}\right|,\left|l_{2}-n_{2}\right|-1\right\}+1 \\
& =d_{21}(l-n)+1 \\
& \leq d_{12}(m-l)+d_{21}(l-n)+1 .
\end{aligned}
$$
viii).

We can treat the other cases of (vil) similarly; also, the cases ( $(\mathbf{v})$, (vii), and

The similar arguments prove the inequality for $(i, j)=(2,1)$.

\section{Appendix D}

\section{D.1 Proof of Lemma 6.1}

Asymptotic behavior of II

We split $I I$ into four parts: $I I=I I_{1}+I I_{2}+I I_{3}+I I_{4}$, where

$$
I_{1}=\sum_{n_{2} \geq p+1} \sum_{m_{2} \geq p+1}, I I_{2}=\sum_{n_{2} \geq p+1} \sum_{m_{2} \leq p}, I I_{3}=\sum_{n_{2} \leq p} \sum_{m_{2} \geq p+1}, I I_{4}=\sum_{n_{2} \leq p} \sum_{m_{2} \leq p} .
$$

About $I I_{4}$, it is a sum of the following terms:

$$
\frac{\bar{\alpha}\left(\zeta_{+}(z, \theta)\right)}{\sqrt{8 z^{2}+1}} e^{i n \zeta_{+}(z, \theta)} \hat{q}_{1}(n) e^{-i m \zeta_{-}\left(z, \theta^{\prime}\right)}\left(\langle\hat{P}(n)|\hat{R}(z)| \hat{P}(m)\rangle \hat{q}(m) \hat{\eta}^{(0)}\left(z, \theta^{\prime}\right)\right)_{12},
$$

where $n_{2} \leq p$ and $m_{2} \leq p$. Then, by using (82), (77), (78), and (88), we have $I I_{4} \sim \mathcal{O}\left(N^{2\left(n_{2}+m_{2}\right)-3}\right) \sim \mathcal{O}\left(N^{4 p-3}\right)$. 
About $I I_{3}$, we note that it is a sum of (119)'s, where $n_{2} \leq p$ and $m_{2} \geq p+1$. By using (87), we have

$$
\begin{aligned}
& \left(\langle\hat{P}(n)|\hat{R}(z)| \hat{P}(m)\rangle \hat{q}(m) \hat{\eta}\left(z, \theta^{\prime}\right)\right)_{12} \\
& =\mathcal{O}\left(\langle z\rangle^{-2 d(n-m)-1}\right) \hat{q}_{1}(m) \frac{\alpha\left(\zeta_{-}\left(z, \theta^{\prime}\right)\right)}{\sqrt{8 z^{2}+1}}+\mathcal{O}\left(\langle z\rangle^{-2 d_{12}(n-m)-2}\right) \hat{q}_{2}(m) .
\end{aligned}
$$

From Lemma 5.1, we have

$$
d_{12}(n-m) \geq\left|n_{2}-m_{2}\right|
$$

since $n_{2}-m_{2} \leq 0 ;$ also,

$$
d(n-m) \geq\left|n_{2}-m_{2}\right|
$$

Then, by using (83), (121), and (122), we have $\mathcal{O}\left(N^{-2\left|n_{2}-m_{2}\right|-2}\right)$ as the asymptotic behavior of (120). Moreover, by using (82), (177), and (78), we have $I I_{3} \sim \mathcal{O}\left(N^{2\left(n_{2}+m_{2}\right)-2\left|n_{2}-m_{2}\right|-3}\right)$. Since

$$
\begin{aligned}
2\left(n_{2}+m_{2}\right)-2\left|n_{2}-m_{2}\right| & =2\left(\left(m_{2}-n_{2}\right)-\left|n_{2}-m_{2}\right|\right)+4 n_{2} \\
& \leq 4 n_{2},
\end{aligned}
$$

we have $I I_{3} \sim \mathcal{O}\left(N^{4 p-3}\right)$.

About $I I_{2}$, we note that

$$
d_{12}(n-m) \geq\left|n_{2}-m_{2}\right|-1
$$

since $n_{2}-m_{2} \geq 0$, which infers $I I_{2} \sim \mathcal{O}\left(N^{4 p-1}\right)$ in the same way as $I I_{3}$.

About $I I_{1}$, by using the resolvent equation

$$
\hat{R}(z)=\hat{R}_{>p}(z)-\hat{R}_{>p}(z) \hat{q}_{\leq p} \hat{R}(z)
$$

we split it into two parts: $I I_{1}=I I_{1,1}-I I_{1,2}$, where

$$
\begin{aligned}
& I I_{1,1}=\frac{\bar{\alpha}\left(\zeta_{+}(z, \theta)\right)}{\sqrt{8 z^{2}+1}} \sum_{n_{2} \geq p+1} e^{i n \zeta_{+}(z, \theta)} \hat{q}_{1}(n) \sum_{m_{2} \geq p+1} e^{-i m \zeta_{-}\left(z, \theta^{\prime}\right)} \\
& \cdot\left(\left\langle\hat{P}(n)\left|\hat{R}_{>p}(z)\right| \hat{P}(m)\right\rangle \hat{q}(m) \hat{\eta}^{(0)}\left(z, \theta^{\prime}\right)\right)_{12} \text {, } \\
& I I_{1,2}=\frac{\bar{\alpha}\left(\zeta_{+}(z, \theta)\right)}{\sqrt{8 z^{2}+1}} \sum_{n_{2} \geq p+1} e^{i n \zeta_{+}(z, \theta)} \hat{q}_{1}(n) \sum_{m_{2} \geq p+1} e^{-i m \zeta_{-}\left(z, \theta^{\prime}\right)} \\
& \cdot\left(\left\langle\hat{P}(n)\left|\hat{R}_{>p}(z) \hat{q}_{\leq p} \hat{R}(z)\right| \hat{P}(m)\right\rangle \hat{q}(m) \hat{\eta}^{(0)}\left(z, \theta^{\prime}\right)\right)_{12} \text {. }
\end{aligned}
$$

We know $\hat{R}_{>p}(z)$, since we have already computed $\hat{q}(n)$ for $n_{2}>p$ by the assumption, which means that $I I_{1,1}$ is a known term. 
$I I_{1,2}$ is a sum of the following terms:

$$
\begin{aligned}
& \frac{\bar{\alpha}\left(\zeta_{+}(z, \theta)\right)}{\sqrt{8 z^{2}+1}} e^{i n \zeta_{+}(z, \theta)} \hat{q}_{1}(n) e^{-i m \zeta_{-}\left(z, \theta^{\prime}\right)} \\
& \cdot\left(\left\langle\hat{P}(n)\left|\hat{R}_{>p}(z)\right| \hat{P}(k)\right\rangle \hat{q}(k)\langle\hat{P}(k)|\hat{R}(z)| \hat{P}(m)\rangle \hat{q}(m) \hat{\eta}^{(0)}\left(z, \theta^{\prime}\right)\right)_{12},
\end{aligned}
$$

where $n_{2} \geq p+1, k_{2} \leq p$, and $m_{2} \geq p+1$. By using (73), (8), and (87), we have

$$
\begin{aligned}
&\left(\left\langle\hat{P}(n)\left|\hat{R}_{>p}(z)\right| \hat{P}(k)\right\rangle \hat{q}(k)\langle\hat{P}(k)|\hat{R}(z)| \hat{P}(m)\rangle \hat{q}(m) \hat{\eta}^{(0)}\left(z, \theta^{\prime}\right)\right)_{12} \\
&= \mathcal{O}\left(\langle z\rangle^{-2 d(n-k)-1}\right) \hat{q}_{1}(k) \mathcal{O}\left(\langle z\rangle^{-2 d(k-m)-1}\right) \hat{q}_{1}(m) \frac{\alpha\left(\zeta_{-}\left(z, \theta^{\prime}\right)\right)}{\sqrt{8 z^{2}+1}} \\
&+\mathcal{O}\left(\langle z\rangle^{-2 d(n-k)-1}\right) \hat{q}_{1}(k) \mathcal{O}\left(\langle z\rangle^{-2 d_{12}(k-m)-2}\right) \hat{q}_{2}(m) \\
&+\mathcal{O}\left(\langle z\rangle^{-2 d_{12}(n-k)-2}\right) \hat{q}_{2}(k) \mathcal{O}\left(\langle z\rangle^{-2 d_{21}(k-m)-2}\right) \hat{q}_{1}(m) \frac{\alpha\left(\zeta_{-}\left(z, \theta^{\prime}\right)\right)}{\sqrt{8 z^{2}+1}} \\
&+\mathcal{O}\left(\langle z\rangle^{-2 d_{12}(n-k)-2}\right) \hat{q}_{2}(k) \mathcal{O}\left(\langle z\rangle^{-2 d(k-m)-1}\right) \hat{q}_{2}(m) .
\end{aligned}
$$

From Lemma 5.1, we have

$$
\begin{gathered}
d_{12}(n-k) \geq\left|n_{2}-k_{2}\right|-1, \\
d_{12}(k-n) \geq\left|k_{2}-n_{2}\right|, \\
d_{21}(k-m) \geq\left|k_{2}-m_{2}\right|-1,
\end{gathered}
$$

since $k_{2}-m_{2}<0$ and $n_{2}-k_{2}>0 ;$ also,

$$
\begin{gathered}
d(n-k) \geq\left|n_{2}-k_{2}\right|, \\
d(k-m) \geq\left|k_{2}-m_{2}\right| .
\end{gathered}
$$

Then, from Lemmas 5.3, 5.4 and 5.5, by using (83), (126), (127), (128), (129), and (130), we have $\mathcal{O}\left(N^{-2 \mid}\left|n_{2}-k_{2}\right|-2\left|k_{2}-m_{2}\right|-1\right)$ as the asymptotic behavior of (125). Moreover, by using (82), (177), and (78), we have $I I_{1,2} \sim \mathcal{O}\left(N^{2\left(n_{2}+m_{2}\right)-2\left|n_{2}-k_{2}\right|-2\left|k_{2}-m_{2}\right|-2}\right)$. Since

$$
\begin{aligned}
2\left(n_{2}-m_{2}\right)-2\left|n_{2}-k_{2}\right|-2\left|k_{2}-m_{2}\right|= & 2\left(\left(n_{2}-k_{2}\right)-\left|n_{2}-k_{2}\right|\right) \\
& +2\left(\left(m_{2}-k_{2}\right)-\left|m_{2}-k_{2}\right|\right)+4 k_{2} \\
\leq & 4 k_{2},
\end{aligned}
$$

we have $I I_{1,2} \sim \mathcal{O}\left(N^{4 p-2}\right)$.

\section{Asymptotic behavior of III}

We split $I I I$ into four parts: $I I I=I I I_{1}+I I I_{2}+I I I_{3}+I I I_{4}$, where

$$
I I I_{1}=\sum_{n_{2} \geq p+1} \sum_{m_{2} \geq p+1}, I I I_{2}=\sum_{n_{2} \geq p+1} \sum_{m_{2} \leq p}, I I I_{3}=\sum_{n_{2} \leq p} \sum_{m_{2} \geq p+1}, I I I_{4}=\sum_{n_{2} \leq p} \sum_{m_{2} \leq p} .
$$


About $I I I_{4}$, it is a sum of the following terms:

$$
e^{i n \zeta_{+}(z, \theta)} \hat{q}_{2}(n) e^{-i m \zeta_{-}\left(z, \theta^{\prime}\right)}\left(\langle\hat{P}(n)|\hat{R}(z)| \hat{P}(m)\rangle \hat{q}(m) \hat{\eta}^{(0)}\left(z, \theta^{\prime}\right)\right)_{22},
$$

where $n_{2} \leq p$ and $m_{2} \leq p$. Then, by using (77), (78), and (88), we have $I I I_{4} \sim \mathcal{O}\left(N^{2\left(n_{2}+m_{2}\right)-1}\right) \sim \mathcal{O}\left(N^{4 p-1}\right)$.

About $I I I_{3}$, it is a sum of (132)'s, where $n_{2} \leq p$ and $m_{2} \geq p+1$. By using (87), we have

$$
\begin{aligned}
& \left(\langle\hat{P}(n)|\hat{R}(z)| \hat{P}(m)\rangle \hat{q}(m) \hat{\eta}^{(0)}\left(z, \theta^{\prime}\right)\right)_{22} \\
& =\mathcal{O}\left(\langle z\rangle^{-2 d_{21}(n-m)-2}\right) \hat{q}_{1}(m) \frac{\alpha\left(\zeta_{-}\left(z, \theta^{\prime}\right)\right)}{\sqrt{8 z^{2}+1}}+\mathcal{O}\left(\langle z\rangle^{-2 d(n-m)-1}\right) \hat{q}_{2}(m) .
\end{aligned}
$$

From Lemma 5.1, we have

$$
d_{21}(n-m) \geq\left|n_{2}-m_{2}\right|-1,
$$

since $n_{2}-m_{2} \leq 0 ;$ also,

$$
d(n-m) \geq\left|n_{2}-m_{2}\right| .
$$

Then, by using (83), (134), and (135), we have $\mathcal{O}\left(N^{-2\left|n_{2}-m_{2}\right|-1}\right)$ as the asymptotic behavior of (133). Moreover, by using (77) and (78), we have $I I I_{3} \sim$ $\mathcal{O}\left(N^{2\left(n_{2}+m_{2}\right)-2\left|n_{2}-m_{2}\right|-1}\right)$. The estimate (123) implies $I I I_{3} \sim \mathcal{O}\left(N^{4 p-1}\right)$.

About $I I I_{2}$, we note that

$$
d_{21}(n-m) \geq\left|n_{2}-m_{2}\right|
$$

since $n_{2}-m_{2} \geq 0$, which infers $I I I_{2} \sim \mathcal{O}\left(N^{4 p-1}\right)$ in the same way as $I I I_{3}$.

About $I I I_{1}$, by using the resolvent estimate (124), we split it into two parts: $I I I_{1}=I I I_{1,1}-I I I_{1,2}$, where

$$
\begin{gathered}
I I I_{1,1}=\sum_{n_{2} \geq p+1} e^{i n \zeta_{+}(z, \theta)} \hat{q}_{2}(n) \sum_{m_{2} \geq p+1} e^{-i m \zeta_{-}\left(z, \theta^{\prime}\right)} \\
\cdot\left(\left\langle\hat{P}(n)\left|\hat{R}_{>p}(z)\right| \hat{P}(m)\right\rangle \hat{q}(m) \hat{\eta}^{(0)}\left(z, \theta^{\prime}\right)\right)_{22}, \\
I I I_{1,2}=\sum_{n_{2} \geq p+1} e^{i n \zeta_{+}(z, \theta)} \hat{q}_{2}(n) \sum_{m_{2} \geq p+1} e^{-i m \zeta_{-}\left(z, \theta^{\prime}\right)} \\
\cdot\left(\left\langle\hat{P}(n)\left|\hat{R}_{>p}(z) \hat{q}_{\leq p} \hat{R}(z)\right| \hat{P}(m)\right\rangle \hat{q}(m) \hat{\eta}^{(0)}\left(z, \theta^{\prime}\right)\right)_{22} .
\end{gathered}
$$

$I I I_{1,1}$ is a known term as before.

$I I I_{1,2}$ is a sum of the following terms:

$$
\begin{aligned}
& e^{i n \zeta_{+}(z, \theta)} \hat{q}_{2}(n) e^{-i m \zeta_{-}\left(z, \theta^{\prime}\right)} \\
& \cdot\left(\left\langle\hat{P}(n)\left|\hat{R}_{>p}(z)\right| \hat{P}(k)\right\rangle \hat{q}(k)\langle\hat{P}(k)|\hat{R}(z)| \hat{P}(m)\rangle \hat{q}(m) \hat{\eta}^{(0)}\left(z, \theta^{\prime}\right)\right)_{22},
\end{aligned}
$$


where $n_{2} \geq p+1, k_{2} \leq p$, and $m_{2} \geq p+1$. By using (73), (87), and (87), we have

$$
\begin{aligned}
\left(\left\langle\hat{P}(n)\left|\hat{R}_{>p}(z)\right| \hat{P}(k)\right\rangle \hat{q}(k)\langle\hat{P}(k)|\hat{R}(z)| \hat{P}(m)\rangle \hat{q}(m) \hat{\eta}^{(0)}\left(z, \theta^{\prime}\right)\right)_{22} \\
=\mathcal{O}\left(\langle z\rangle^{-2 d_{21}(n-k)-2}\right) \hat{q}_{1}(k) \mathcal{O}\left(\langle z\rangle^{-2 d(k-m)-1}\right) \hat{q}_{1}(m) \frac{\alpha\left(\zeta_{-}\left(z, \theta^{\prime}\right)\right)}{\sqrt{8 z^{2}+1}} \\
\quad+\mathcal{O}\left(\langle z\rangle^{-2 d_{21}(n-k)-2}\right) \hat{q}_{1}(k) \mathcal{O}\left(\langle z\rangle^{-2 d_{12}(k-m)-2}\right) \hat{q}_{2}(m) \\
\quad+\mathcal{O}\left(\langle z\rangle^{-2 d(n-k)-1}\right) \hat{q}_{2}(k) \mathcal{O}\left(\langle z\rangle^{-2 d_{21}(k-m)-2}\right) \hat{q}_{1}(m) \frac{\alpha\left(\zeta_{-}\left(z, \theta^{\prime}\right)\right)}{\sqrt{8 z^{2}+1}} \\
\quad+\mathcal{O}\left(\langle z\rangle^{-2 d(n-k)-1}\right) \hat{q}_{2}(k) \mathcal{O}\left(\langle z\rangle^{-2 d(k-m)-1}\right) \hat{q}_{2}(m) .
\end{aligned}
$$

From Lemma 5.1, we have

$$
\begin{gathered}
d_{21}(n-k) \geq\left|n_{2}-k_{2}\right|, \\
d_{21}(k-m) \geq\left|k_{2}-m_{2}\right|-1, \\
d_{12}(k-m) \geq\left|k_{2}-m_{2}\right|,
\end{gathered}
$$

since $n_{2}-k_{2}>0$ and $k_{2}-m_{2}<0$; also,

$$
\begin{gathered}
d(n-k) \geq\left|n_{2}-k_{2}\right|, \\
d(k-m) \geq\left|k_{2}-m_{2}\right| .
\end{gathered}
$$

Then, by using (83), (137), (138), (139), (140), and (141), we have $\mathcal{O}\left(N^{\left.-2\left|n_{2}-k_{2}\right|-2\left|k_{2}-m_{2}\right|-2\right)}\right.$ as the asymptotic behavior of (136). Moreover, by using (77) and (78), we have $I I I_{1,2} \sim \mathcal{O}\left(N^{\left.2\left(n_{2}+m_{2}\right)-2\left|n_{2}-k_{2}\right|-2\left|k_{2}-m_{2}\right|-2\right)}\right.$. The estimate (131) implies $I I I_{1,2} \sim \mathcal{O}\left(N^{4 p-2}\right)$.

\section{D.2 Proof of Lemma 6.2}

Asymptotic behavior of $I V$

We split $I V$ into four parts: $I V=I V_{1}+I V_{2}+I V_{3}+I V_{4}$, where

$$
I V_{1}=\sum_{n_{2} \geq p+1} \sum_{m_{2} \geq p+1}, I V_{2}=\sum_{n_{2} \geq p+1} \sum_{m_{2} \leq p}, I V_{3}=\sum_{n_{2} \leq p} \sum_{m_{2} \geq p+1}, I V_{4}=\sum_{n_{2} \leq p} \sum_{m_{2} \leq p} .
$$

About $I V_{4}$, it is a sum of the following terms:

$$
e^{i n \zeta_{+}(z, \theta)} \hat{q}_{1}(n) e^{-i m \zeta_{-}\left(z, \theta^{\prime}\right)}\left(\langle\hat{P}(n)|\hat{R}(z)| \hat{P}(m)\rangle \hat{q}(m) \hat{\eta}^{(0)}\left(z, \theta^{\prime}\right)\right)_{11},
$$

where $n_{2} \leq p$ and $m_{2} \leq p$. Then, by using (77), (78), and (88), we have $I V_{4} \sim \mathcal{O}\left(N^{2}\left(n_{2}+m_{2}\right)-1\right)=\mathcal{O}\left(N^{4 p-1}\right)$.

About $I V_{3}$, it is a sum of (142)'s, where $n_{2} \leq p$ and $m_{2} \geq p+1$. By using (87), we have

$$
\begin{aligned}
& \left(\langle\hat{P}(n)|\hat{R}(z)| \hat{P}(m)\rangle \hat{q}(m) \hat{\eta}^{(0)}\left(z, \theta^{\prime}\right)\right)_{11} \\
& =\mathcal{O}\left(\langle z\rangle^{-2 d(n-m)-1}\right) \hat{q}_{1}(m)+\mathcal{O}\left(\langle z\rangle^{-2 d_{12}(n-m)-2}\right) \hat{q}_{2}(m) \frac{\bar{\alpha}\left(\zeta_{-}\left(z, \theta^{\prime}\right)\right)}{\sqrt{8 z^{2}+1}} .
\end{aligned}
$$


From Lemma 5.1, we have

$$
d_{12}(n-m) \geq\left|n_{2}-m_{2}\right|
$$

since $n_{2}-m_{2}<0 ;$ also,

$$
d(n-m) \geq\left|n_{2}-m_{2}\right| .
$$

Then, by using (84), (144), and (145), we have $\mathcal{O}\left(N^{-2\left|n_{2}-m_{2}\right|-1}\right)$ as the asymptotic behavior of (143). Moreover, by using (77) and (78), we have $I V_{3} \sim$ $\mathcal{O}\left(N^{2\left(n_{2}+m_{2}\right)-2\left|n_{2}-m_{2}\right|-1}\right)$. The estimate (123) implies $I_{3} \sim \mathcal{O}\left(N^{4 p-1}\right)$.

About $I V_{2}$, by using the resolvent equation

$$
\hat{R}(z)=\hat{R}_{(>p,>p-1)}(z)-\hat{R}_{(>p,>p-1)}(z) \hat{q}_{(\leq p, \leq p-1)} \hat{R}(z),
$$

we split it into five parts: $I V_{2}=I V_{2,1}+I V_{2,2,1}+I V_{2,2,2,1}-I V_{2,2,2,2,1}-I V_{2,2,2,2,2}$, where

$$
\begin{aligned}
& I V_{2,1}=\sum_{n_{2} \geq p+1} e^{i n \zeta_{+}(z, \theta)} \hat{q}_{1}(n) \sum_{m_{2} \leq p-1} e^{-i m \zeta_{-}\left(z, \theta^{\prime}\right)} \\
& \cdot\left(\langle\hat{P}(n)|\hat{R}(z)| \hat{P}(m)\rangle \hat{q}(m) \hat{\eta}^{(0)}\left(z, \theta^{\prime}\right)\right)_{11} \text {, } \\
& I V_{2,2,1}=\sum_{n_{2} \geq p+1} e^{i n \zeta_{+}(z, \theta)} \hat{q}_{1}(n) \sum_{m_{2}=p} e^{-i m \zeta_{-}\left(z, \theta^{\prime}\right)} \\
& \cdot\left(\langle\hat{P}(n)|\hat{R}(z)| \hat{P}(m)\rangle\left(\begin{array}{cc}
\hat{q}_{1}(m) & 0 \\
0 & 0
\end{array}\right) \hat{\eta}^{(0)}\left(z, \theta^{\prime}\right)\right)_{11}, \\
& I V_{2,2,2,1}=\sum_{n_{2} \geq p+1} e^{i n \zeta_{+}(z, \theta)} \hat{q}_{1}(n) \sum_{m_{2}=p} e^{-i m \zeta_{-}\left(z, \theta^{\prime}\right)} \\
& \cdot\left(\left\langle\hat{P}(n)\left|\hat{R}_{(>p,>p-1)}(z)\right| \hat{P}(m)\right\rangle\left(\begin{array}{cc}
0 & 0 \\
0 & \hat{q}_{2}(m)
\end{array}\right) \hat{\eta}^{(0)}\left(z, \theta^{\prime}\right)\right)_{11}, \\
& I V_{2,2,2,2,1}=\sum_{n_{2} \geq p+1} e^{i n \zeta_{+}(z, \theta)} \hat{q}_{1}(n) \sum_{m_{2}=p} e^{-i m \zeta_{-}\left(z, \theta^{\prime}\right)} \\
& \cdot\left(\left\langle\hat{P}(n)\left|\hat{R}_{(>p,>p-1)}(z) \hat{q}_{\leq p-1} \hat{R}(z)\right| \hat{P}(m)\right\rangle\left(\begin{array}{cc}
0 & 0 \\
0 & \hat{q}_{2}(m)
\end{array}\right) \hat{\eta}^{(0)}\left(z, \theta^{\prime}\right)\right)_{11}, \\
& I V_{2,2,2,2,2}=\sum_{n_{2} \geq p+1} e^{i n \zeta_{+}(z, \theta)} \hat{q}_{1}(n) \sum_{m_{2}=p} e^{-i m \zeta_{-}\left(z, \theta^{\prime}\right)} \\
& \cdot \sum_{k_{2}=p}\left(\left\langle\hat{P}(n)\left|\hat{R}_{(>p,>p-1)}(z) \hat{P}(k)\left(\begin{array}{cc}
\hat{q}_{1}(k) & 0 \\
0 & 0
\end{array}\right) \hat{P}(k) \hat{R}(z)\right| \hat{P}(m)\right\rangle\right. \\
& \left.\cdot\left(\begin{array}{cc}
0 & 0 \\
0 & \hat{q}_{2}(m)
\end{array}\right) \hat{\eta}^{(0)}\left(z, \theta^{\prime}\right)\right)_{11} .
\end{aligned}
$$


$I V_{2,1}$ is a sum of (142)'s, where $n_{2} \geq p+1$ and $m_{2} \leq p-1$. From Lemma 5.1. we have

$$
d_{12}(n-m) \geq\left|n_{2}-m_{2}\right|-1,
$$

since $n_{2}-m_{2}>0$. Then, by using (84) and (147), we have $I V_{2,1} \sim \mathcal{O}\left(N^{4 p-3}\right)$ in the same way as $I V_{3}$.

$I V_{2,2,1}$ is also a sum of (142)'s, where $n_{2} \geq p+1$ and $m_{2}=p$. Then, by noticing that $\hat{q}_{2}(m)=0$ for $m_{2}=p$, we have $I V_{2,2,1} \sim \mathcal{O}\left(N^{4 p-1}\right)$ in the same way as above.

We know $\hat{R}_{(>p,>p-1)}(z)$, since we have already computed $\hat{q}(n)$ for $n_{2} \geq p+1$ and $\hat{q}_{2}(n)$ for $n_{2}=p$, which implies that $I V_{2,2,2,1}$ is a known term.

$I V_{2,2,2,2,1}$ is a linear combination of the following terms:

$$
\begin{aligned}
& e^{i n \zeta_{+}(z, \theta)} e^{-i m \zeta_{-}\left(z, \theta^{\prime}\right)} \\
& \cdot\left(\left\langle\hat{P}(n)\left|\hat{R}_{(>p,>p-1)}(z)\right| \hat{P}(k)\right\rangle \hat{q}(k)\langle\hat{P}(k)|\hat{R}(z)| \hat{P}(m)\rangle\left(\begin{array}{cc}
0 & 0 \\
0 & 1
\end{array}\right) \hat{\eta}^{(0)}\left(z, \theta^{\prime}\right)\right)_{11},
\end{aligned}
$$

where $n_{2} \geq p+1, k_{2} \leq p-1$, and $m_{2}=p$. By using (87), we have

$$
\begin{aligned}
& \left(\left\langle\hat{P}(n)\left|\hat{R}_{(>p,>p-1)}(z)\right| \hat{P}(k)\right\rangle \hat{q}(k)\langle\hat{P}(k)|\hat{R}(z)| \hat{P}(m)\rangle\left(\begin{array}{cc}
0 & 0 \\
0 & 1
\end{array}\right) \hat{\eta}^{(0)}\left(z, \theta^{\prime}\right)\right)_{11} \\
& =\mathcal{O}\left(\langle z\rangle^{-2 d(n-k)-1}\right) \hat{q}_{1}(k) \mathcal{O}\left(\langle z\rangle^{-2 d_{12}(k-m)-2}\right) \frac{\bar{\alpha}\left(\zeta_{-}\left(z, \theta^{\prime}\right)\right)}{\sqrt{8 z^{2}+1}} \\
& \quad+\mathcal{O}\left(\langle z\rangle^{-2 d_{12}(n-k)-2}\right) \hat{q}_{2}(k) \mathcal{O}\left(\langle z\rangle^{-2 d(k-m)-1}\right) \frac{\bar{\alpha}\left(\zeta_{-}\left(z, \theta^{\prime}\right)\right)}{\sqrt{8 z^{2}+1}} .
\end{aligned}
$$

From Lemma 5.1, we have

$$
\begin{gathered}
d_{12}(k-m) \geq\left|k_{2}-m_{2}\right|, \\
d_{12}(n-k) \geq\left|n_{2}-k_{2}\right|-1,
\end{gathered}
$$

since $k_{2}-m_{2}<0$ and $n_{2}-k_{2}>0$; also,

$$
d(n-k) \geq\left|n_{2}-k_{2}\right| .
$$

Then, from Lemmas 5.3, 5.4, and 5.5) by using (84), (150), (151), and (152), we have $\mathcal{O}\left(N^{-2\left|n_{2}-k_{2}\right|-2\left|k_{2}-m_{2}\right|}\right)$ as the asymptotic behavior of (149). Moreover, by using (77) and (78), we have $I V_{2,2,2,2,1} \sim \mathcal{O}\left(N^{2\left(n_{2}+m_{2}\right)-2\left|n_{2}-k_{2}\right|-2\left|k_{2}-m_{2}\right|}\right)$. The estimate (131) implies $I V_{2,2,2,2,1} \sim \mathcal{O}\left(N^{4 p-4}\right)$.

$I V_{2,2,2,2,2}$ is a linear combination of (148)'s, where $n_{2} \geq p+1, k_{2}=p$, and $m_{2}=p$. Then, by noticing $\hat{q}_{2}(k)=0$ for $k_{2}=p$, we have $I V_{2,2,2,2,2} \sim \mathcal{O}\left(N^{4 p-2}\right)$ in the same way as $I V_{2,2,2,2,1}$.

About $I V_{1}$, by using the resolvent equation (146), we split it into three parts: $I V_{1}=I V_{1,1}-I V_{1,2,1}-I V_{1,2,2}$, where

$$
\begin{aligned}
I V_{1,1}= & \sum_{n_{2} \geq p+1} e^{i n \zeta_{+}(z, \theta)} \hat{q}_{1}(n) \sum_{m_{2} \geq p+1} e^{-i m \zeta_{-}\left(z, \theta^{\prime}\right)} \\
& \cdot\left(\left\langle\hat{P}(n)\left|\hat{R}_{(>p,>p-1)}(z)\right| \hat{P}(m)\right\rangle \hat{q}(m) \hat{\eta}^{(0)}\left(z, \theta^{\prime}\right)\right)_{11},
\end{aligned}
$$




$$
\begin{aligned}
I V_{1,2,1}= & \sum_{n_{2} \geq p+1} e^{i n \zeta_{+}(z, \theta)} \hat{q}_{1}(n) \sum_{m_{2} \geq p+1} e^{-i m \zeta_{-}\left(z, \theta^{\prime}\right)} \\
& \cdot\left(\left\langle\hat{P}(n)\left|\hat{R}_{(>p,>p-1)}(z) \hat{q}_{\leq p-1} \hat{R}(z)\right| \hat{P}(m)\right\rangle \hat{q}(m) \hat{\eta}^{(0)}\left(z, \theta^{\prime}\right)\right)_{11}, \\
I V_{1,2,2}= & \sum_{n_{2} \geq p+1} e^{i n \zeta_{+}(z, \theta)} \hat{q}_{1}(n) \sum_{m_{2} \geq p+1} e^{-i m \zeta_{-}\left(z, \theta^{\prime}\right)} \\
& \cdot \sum_{k_{2}=p}\left(\left\langle\hat{P}(n)\left|\hat{R}_{(>p,>p-1)}(z) \hat{P}(k)\left(\begin{array}{cc}
\hat{q}_{1}(k) & 0 \\
0 & 0
\end{array}\right) \hat{P}(k) \hat{R}(z)\right| \hat{P}(m)\right\rangle\right. \\
& \left.\cdot \hat{q}(m) \hat{\eta}^{(0)}\left(z, \theta^{\prime}\right)\right)_{11} \cdot
\end{aligned}
$$

$I V_{1,1}$ is a known term as before.

$I V_{1,2,1}$ is a linear combination of (148)'s, where $n_{2} \geq p+1, k_{2} \leq p-1$, and $m_{2} \geq p+1$. Then we have $I_{1,2,1} \sim \mathcal{O}\left(N^{4 p-4}\right)$ in the same way as $I_{2,2,2,2,1}$.

$I V_{1,2,2}$ is also a linear combination of (148)'s, where $n_{2} \geq p+1, k_{2}=p$, and $m_{2} \geq p+1$. Then, by noticing $\hat{q}_{2}(k)=0$ for $k_{2}=p$, we have $I_{1,2,2} \sim \mathcal{O}\left(N^{4 p-2}\right)$ in the same way as above.

\section{Asymptotic behavior of $V$}

We split $V$ into four parts: $V=V_{1}+V_{2}+V_{3}+V_{4}$, where

$$
V_{1}=\sum_{n_{2} \geq p} \sum_{m_{2} \geq p+1}, V_{2}=\sum_{n_{2} \geq p} \sum_{m_{2} \leq p}, V_{3}=\sum_{n_{2} \leq p-1} \sum_{m_{2} \geq p+1}, V_{4}=\sum_{n_{2} \leq p-1} \sum_{m_{2} \leq p} .
$$

About $V_{4}$, it is a linear combination of the following terms:

$$
\frac{\alpha\left(\zeta_{+}(z, \theta)\right)}{\sqrt{8 z^{2}+1}} e^{i n \zeta_{+}(z, \theta)} e^{-i m \zeta_{-}\left(z, \theta^{\prime}\right)}\left(\langle\hat{P}(n)|\hat{R}(z)| \hat{P}(m)\rangle \hat{q}(m) \hat{\eta}^{(0)}\left(z, \theta^{\prime}\right)\right)_{21},
$$

where $n_{2} \leq p-1$ and $m_{2} \leq p$. Then, by using (81), (77), (78), and (88), we have $V_{4} \sim \mathcal{O}\left(N^{2\left(n_{2}+m_{2}\right)+1}\right) \sim \mathcal{O}\left(N^{4 p-1}\right)$.

About $V_{3}$, it is a linear combination of (153)'s, where $n_{2} \leq p-1$ and $m_{2} \geq$ $p+1$. By using (87), we have

$$
\begin{aligned}
& \left(\langle\hat{P}(n)|\hat{R}(z)| \hat{P}(m)\rangle \hat{q}(m) \hat{\eta}^{(0)}\left(z, \theta^{\prime}\right)\right)_{21} \\
& \sim \mathcal{O}\left(\langle z\rangle^{-2 d_{21}(n-m)-2}\right) \hat{q}_{1}(m)+\mathcal{O}\left(\langle z\rangle^{-2 d(n-m)-1}\right) \hat{q}_{2}(m) \frac{\bar{\alpha}\left(\zeta_{-}\left(z, \theta^{\prime}\right)\right)}{\sqrt{8 z^{2}+1}} .
\end{aligned}
$$

From Lemma 5.1, we have

$$
d_{21}(n-m) \geq\left|n_{2}-m_{2}\right|-1,
$$

since $n_{2}-m_{2}<0$; also,

$$
d(n-m) \geq\left|n_{2}-m_{2}\right| .
$$


Then, by using (84), (155), and (156), we have $\mathcal{O}\left(N^{-2\left|n_{2}-m_{2}\right|}\right)$ as the asymptotic behavior of (154). Moreover, by using (77), (78), and (81), we have $V_{3} \sim$ $\mathcal{O}\left(N^{2\left(n_{2}+m_{2}\right)-2\left|n_{2}-m_{2}\right|+1}\right)$. The estimate (123) implies $V_{3} \sim \mathcal{O}\left(N^{4 p-3}\right)$.

About $V_{2}$, by using the resolvent equation (146), we split it into five parts: $V_{2}=V_{2,1}+V_{2,2,1}+V_{2,2,2,1}-V_{2,2,2,2,1}-V_{2,2,2,2,2}$, where

$$
\begin{aligned}
& V_{2,1}=\frac{\alpha\left(\zeta_{+}(z, \theta)\right)}{\sqrt{8 z^{2}+1}} \sum_{n_{2} \geq p} e^{i n \zeta_{+}(z, \theta)} \hat{q}_{2}(n) \sum_{m_{2} \leq p-1} e^{-m \zeta_{-}\left(z, \theta^{\prime}\right)} \\
& \cdot\left(\langle\hat{P}(n)|\hat{R}(z)| \hat{P}(m)\rangle \hat{q}(m) \hat{\eta}^{(0)}\left(z, \theta^{\prime}\right)\right)_{21} \text {, } \\
& V_{2,2,1}=\frac{\alpha\left(\zeta_{+}(z, \theta)\right)}{\sqrt{8 z^{2}+1}} \sum_{n_{2} \geq p} e^{i n \zeta_{+}(z, \theta)} \hat{q}_{2}(n) \sum_{m_{2}=p} e^{-i m \zeta_{-}\left(z, \theta^{\prime}\right)} \\
& \cdot\left(\langle\hat{P}(n)|\hat{R}(z)| \hat{P}(m)\rangle\left(\begin{array}{cc}
\hat{q}_{1}(m) & 0 \\
0 & 0
\end{array}\right) \hat{\eta}^{(0)}\left(z, \theta^{\prime}\right)\right)_{21} \text {, } \\
& V_{2,2,2,1}=\frac{\alpha\left(\zeta_{+}(z, \theta)\right)}{\sqrt{8 z^{2}+1}} \sum_{n_{2} \geq p} e^{i n \zeta_{+}(z, \theta)} \hat{q}_{2}(n) \sum_{m_{2}=p} e^{-i m \zeta_{-}\left(z, \theta^{\prime}\right)} \\
& \cdot\left(\left\langle\hat{P}(n)\left|\hat{R}_{(>p,>p-1)}(z)\right| \hat{P}(m)\right\rangle\left(\begin{array}{cc}
0 & 0 \\
0 & \hat{q}_{2}(m)
\end{array}\right) \hat{\eta}^{(0)}\left(z, \theta^{\prime}\right)\right)_{21}, \\
& V_{2,2,2,2,1}=\frac{\alpha\left(\zeta_{+}(z, \theta)\right)}{\sqrt{8 z^{2}+1}} \sum_{n_{2} \geq p} e^{i n \zeta_{+}(z, \theta)} \hat{q}_{2}(n) \sum_{m_{2}=p} e^{-i m \zeta_{-}\left(z, \theta^{\prime}\right)} \\
& \cdot\left(\left\langle P(n)\left|\hat{R}_{(>p,>p-1)} \hat{q}_{\leq p-1} \hat{R}(z)\right| \hat{P}(m)\right\rangle\left(\begin{array}{cc}
0 & 0 \\
0 & \hat{q}_{2}(m)
\end{array}\right) \hat{\eta}^{(0)}\left(z, \theta^{\prime}\right)\right)_{21}, \\
& V_{2,2,2,2,2}=\frac{\alpha\left(\zeta_{+}(z, \theta)\right)}{\sqrt{8 z^{2}+1}} \sum_{n_{2} \geq p} e^{i n \zeta_{+}(z, \theta)} \hat{q}_{2}(n) \sum_{m_{2}=p} e^{-i m \zeta_{-}\left(z, \theta^{\prime}\right)} \\
& \cdot \sum_{k_{2}=p}\left(\left\langle\hat{P}(n)\left|\hat{R}_{(>p,>p-1)}(z) \hat{P}(k)\left(\begin{array}{cc}
\hat{q}_{1}(k) & 0 \\
0 & 0
\end{array}\right) \hat{P}(k) \hat{R}(z)\right| \hat{P}(m)\right\rangle\right. \\
& \left.\cdot\left(\begin{array}{cc}
0 & 0 \\
0 & \hat{q}_{2}(m)
\end{array}\right) \hat{\eta}^{(0)}\left(z, \theta^{\prime}\right)\right)_{21} .
\end{aligned}
$$

$V_{2,1}$ is a linear combination of (153)'s, where $n_{2} \geq p$ and $m_{2} \leq p-1$. From Lemma 5.1, we have

$$
d_{21}(n-m) \geq\left|n_{2}-m_{2}\right|,
$$

since $n_{2}-m_{2}>0 ;$ also,

$$
d(n-m) \geq\left|n_{2}-m_{2}\right| .
$$


Then, from (157) and (158), we have $V_{2,1} \sim \mathcal{O}\left(N^{2\left(n_{2}+m_{2}\right)-2\left|n_{2}-m_{2}\right|+1}\right)$ in the same say as $V_{3}$. The estimate (123) implies $V_{2,1} \sim \mathcal{O}\left(N^{4 p-3}\right)$.

$V_{2,2,1}$ is a sum of (153)'s, where $n_{2} \geq p$ and $m_{2}=p$. Then, by noticing that $\hat{q}_{2}(m)=0$ for $m_{2}=p$, we have $V_{2,2,1} \sim \mathcal{O}\left(N^{4 p-1}\right)$ in the same way as above.

$V_{2,2,2,1}$ is a known term as before.

$V_{2,2,2,2,1}$ is a linear combination of the following terms:

$$
\begin{aligned}
& \frac{\alpha\left(\zeta_{+}(z, \theta)\right)}{\sqrt{8 z^{2}+1}} e^{i n \zeta_{+}(z, \theta)} e^{-i m \zeta_{-}\left(z, \theta^{\prime}\right)}\left(\left\langle\hat{P}(n)\left|\hat{R}_{(>p,>p-1)}(z)\right| \hat{P}(k)\right\rangle\right. \\
& \left.\cdot \hat{q}(k)\langle\hat{P}(k)|\hat{R}(z)| \hat{P}(m)\rangle\left(\begin{array}{ll}
0 & 0 \\
0 & 1
\end{array}\right) \hat{\eta}^{(0)}\left(z, \theta^{\prime}\right)\right)_{21},
\end{aligned}
$$

where $n_{2} \geq p, k_{2} \leq p-1$, and $m_{2}=p$. By using (73), (8), and (87), we have

$$
\begin{aligned}
& \left(\left\langle\hat{P}(n)\left|\hat{R}_{(>p,>p-1)}(z)\right| \hat{P}(k)\right\rangle \hat{q}(k)\langle\hat{P}(k)|\hat{R}(z)| \hat{P}(m)\rangle\left(\begin{array}{cc}
0 & 0 \\
0 & 1
\end{array}\right) \hat{\eta}^{(0)}\left(z, \theta^{\prime}\right)\right)_{21} \\
& \sim \mathcal{O}\left(\langle z\rangle^{-2 d_{21}(n-k)-2}\right) \hat{q}_{1}(k) \mathcal{O}\left(\langle z\rangle^{-2 d_{12}(k-m)-2}\right) \frac{\bar{\alpha}\left(\zeta_{-}\left(z, \theta^{\prime}\right)\right)}{\sqrt{8 z^{2}+1}} \\
& \quad+\mathcal{O}\left(\langle z\rangle^{-2 d(n-k)-1}\right) \hat{q}_{2}(k) \mathcal{O}\left(\langle z\rangle^{-2 d(k-m)-1}\right) \frac{\bar{\alpha}\left(\zeta_{-}\left(z, \theta^{\prime}\right)\right)}{\sqrt{8 z^{2}+1}} .
\end{aligned}
$$

From Lemma 5.1, we have

$$
\begin{gathered}
d_{21}(n-k) \geq\left|n_{2}-k_{2}\right|, \\
d_{12}(k-m) \geq\left|k_{2}-m_{2}\right|,
\end{gathered}
$$

since $n_{2}-k_{2}>0$ and $k_{2}-m_{2}<0$; also,

$$
\begin{gathered}
d(n-k) \geq\left|n_{2}-k_{2}\right|, \\
d(k-m) \geq\left|k_{2}-m_{2}\right| .
\end{gathered}
$$

Then, by using (84), (161), (162), (163), and (164), we have $\mathcal{O}\left(N^{-2\left|n_{2}-k_{2}\right|-2\left|k_{2}-m_{2}\right|-1}\right)$ as the asymptotic behavior of (160). Moreover, by using (77), (78), and (81), we have $V_{2,2,2,2,1} \sim \mathcal{O}\left(N^{2\left(n_{2}+m_{2}\right)-2\left|n_{2}-k_{2}\right|-2\left|k_{2}-m_{2}\right|}\right)$. The estimate (131) implies $V_{2,2,2,2,1} \sim \mathcal{O}\left(N^{4 p-4}\right)$.

$V_{2,2,2,2,2}$ is a linear combination of (159)'s, where $n_{2} \geq p, k_{2}=p$, and $m_{2}=p$. Then, by noticing $\hat{q}_{2}(k)=0$ for $k_{2}=p$, we have $V_{2,2,2,2,2} \sim \mathcal{O}\left(N^{4 p-2}\right)$ in the same as way as $V_{2,2,2,2,1}$.

About $V_{1}$, by using the resolvent equation (146), we split it into three parts: $V_{1}=V_{1,1}-V_{1,2,1}-V_{1,2,2}$, where

$$
\begin{aligned}
V_{1,1}= & \frac{\alpha\left(\zeta_{+}(z, \theta)\right)}{\sqrt{8 z^{2}+1}} \sum_{n_{2} \geq p} e^{i n \zeta_{+}(z, \theta)} \hat{q}_{2}(n) \sum_{m_{2} \geq p+1} e^{-i m \zeta_{-}\left(z, \theta^{\prime}\right)} \\
& \cdot\left(\left\langle\hat{P}(n)\left|\hat{R}_{(>p,>p-1)}(z)\right| \hat{P}(m)\right\rangle \hat{q}(m) \hat{\eta}^{(0)}\left(z, \theta^{\prime}\right)\right)_{21}
\end{aligned}
$$




$$
\begin{aligned}
V_{1,2,1}= & \frac{\alpha\left(\zeta_{+}(z, \theta)\right)}{\sqrt{8 z^{2}+1}} \sum_{n_{2} \geq p} e^{i n \zeta_{+}(z, \theta)} \hat{q}_{2}(n) \sum_{m_{2} \geq p+1} e^{-i m \zeta_{-}\left(z, \theta^{\prime}\right)} \\
& \cdot\left(\left\langle\hat{P}(n)\left|\hat{R}_{(>p,>p-1)}(z) \hat{q}_{\leq p-1} \hat{R}(z)\right| \hat{P}(m)\right\rangle \hat{q}(m) \hat{\eta}^{(0)}\left(z, \theta^{\prime}\right)\right)_{21}, \\
V_{1,2,2}= & \frac{\alpha\left(\zeta_{+}(z, \theta)\right)}{\sqrt{8 z^{2}+1}} \sum_{n_{2} \geq p} e^{i n \zeta_{+}(z, \theta)} \hat{q}_{2}(n) \sum_{m_{2} \geq p+1} e^{-i m \zeta_{-}\left(z, \theta^{\prime}\right)} \\
& \cdot \sum_{k_{2}=p}\left(\left\langle\hat{P}(n)\left|\hat{R}_{(>p,>p-1)}(z) \hat{P}(k)\left(\begin{array}{cc}
\hat{q}_{1}(k) & 0 \\
0 & 0
\end{array}\right) \hat{P}(k) \hat{R}(z)\right| \hat{P}(m)\right\rangle\right. \\
& \left.\cdot \hat{q}(m) \hat{\eta}^{(0)}\left(z, \theta^{\prime}\right)\right)_{21} .
\end{aligned}
$$

$V_{1,1}$ is a known term as before.

$V_{1,2,1}$ is a sum of the following terms:

$$
\begin{aligned}
& \frac{\alpha\left(\zeta_{+}(z, \theta)\right)}{\sqrt{8 z^{2}+1}} e^{i n \zeta_{+}(z, \theta)} e^{-i m \zeta_{-}\left(z, \theta^{\prime}\right)} \\
& \cdot\left(\left\langle\hat{P}(n)\left|\hat{R}_{(>p,>p-1)}(z)\right| \hat{P}(k)\right\rangle \hat{q}(k)\langle\hat{P}(k)|\hat{R}(z)| \hat{P}(m)\rangle \hat{q}(m) \hat{\eta}^{(0)}\left(z, \theta^{\prime}\right)\right)_{21},
\end{aligned}
$$

where $n_{2} \geq p, k_{2} \leq p-1$, and $m_{2} \geq p+1$. By using (73), (87), and (87), we have

$$
\begin{aligned}
& \left(\left\langle\hat{P}(n)\left|\hat{R}_{(>p,>p-1)}(z)\right| \hat{P}(k)\right\rangle \hat{q}(k)\langle\hat{P}(k)|\hat{R}(z)| \hat{P}(m)\rangle \hat{q}(m) \hat{\eta}^{(0)}\left(z, \theta^{\prime}\right)\right)_{21} \\
& \sim \mathcal{O}\left(\langle z\rangle^{-2 d_{21}(n-k)-2}\right) \hat{q}_{1}(k) \mathcal{O}\left(\langle z\rangle^{-2 d(k-m)-1}\right) \hat{q}_{1}(m) \\
& \quad+\mathcal{O}\left(\langle z\rangle^{-2 d_{21}(n-k)-2}\right) \hat{q}_{1}(k) \mathcal{O}\left(\langle z\rangle^{-2 d_{12}(k-m)-2}\right) \hat{q}_{2}(m) \frac{\bar{\alpha}\left(\zeta_{-}\left(z, \theta^{\prime}\right)\right)}{\sqrt{8 z^{2}+1}} \\
& \quad+\mathcal{O}\left(\langle z\rangle^{-2 d(n-k)-1}\right) \hat{q}_{2}(k) \mathcal{O}\left(\langle z\rangle^{-2 d_{21}(k-m)-2}\right) \hat{q}_{1}(m) \\
& \quad+\mathcal{O}\left(\langle z\rangle^{-2 d(n-k)-1}\right) \hat{q}_{2}(k) \mathcal{O}\left(\langle z\rangle^{-2 d(k-m)-1}\right) \hat{q}_{2}(m) \frac{\bar{\alpha}\left(\zeta_{-}\left(z, \theta^{\prime}\right)\right)}{\sqrt{8 z^{2}+1}}
\end{aligned}
$$

From Lemma 5.1, we have

$$
\begin{gathered}
d_{21}(n-k) \geq\left|n_{2}-k_{2}\right|, \\
d_{12}(k-m) \geq\left|k_{2}-m_{2}\right|, \\
d_{21}(k-m) \geq\left|k_{2}-m_{2}\right|-1,
\end{gathered}
$$

since $n_{2}-k_{2}>0$ and $k_{2}-m_{2}<0$; also,

$$
\begin{gathered}
d(n-k) \geq\left|n_{2}-k_{2}\right|, \\
d(k-m) \geq\left|k_{2}-m_{2}\right| .
\end{gathered}
$$

Then, by using (84), (167), (168), (169), (170), and (171), we have $\mathcal{O}\left(N^{-2\left|n_{2}-k_{2}\right|-2\left|k_{2}-m_{2}\right|-1}\right)$ as the asymptotic behavior of (166). Moreover, by using (81), (777), and (78), we have $V_{1,2,1} \sim \mathcal{O}\left(N^{2\left(n_{2}+m_{2}\right)-2\left|n_{2}-k_{2}\right|-2\left|k_{2}-m_{2}\right|}\right)$. The estimate (131) implies $V_{1,2,1} \sim \mathcal{O}\left(N^{4 p-4}\right)$.

$V_{1,2,2}$ is a linear combination of (165)'s, where $n_{2} \geq p, k_{2}=p$, and $m_{2} \geq$ $p+1$. Then, by noticing $\hat{q}_{2}(k)=0$ for $k_{2}=p$, we have $V_{1,2,2} \sim \mathcal{O}\left(N^{4 p-2}\right)$ in the same way as $V_{1,2,1}$. 


\section{References}

[1] R. Beals and R. R. Coifman. Multidimensional inverse scatterings and nonlinear partial differential equations. In Pseudodifferential operators and applications (Notre Dame, Ind., 1984), volume 43 of Proc. Sympos. Pure Math., pages 45-70. Amer. Math. Soc., Providence, RI, 1985.

[2] K. M. Case and M. Kac. A discrete version of the inverse scattering problem. J. Mathematical Phys., 14:594-603, 1973.

[3] A. H. Castro Neto, F. Guinea, N. M. R. Peres, K. S. Novoselov, and A. K. Geim. The electronic properties of graphene. Rev. Mod. Phys., 81(1):109162, Jan 2009.

[4] Fan R. K. Chung. Spectral graph theory, volume 92 of CBMS Regional Conference Series in Mathematics. Published for the Conference Board of the Mathematical Sciences, Washington, DC, 1997.

[5] L. D. Faddeev. Uniqueness of solution of the inverse scattering problem. Vestnik Leningrad. Univ., 11(7):126-130, 1956.

[6] L. D. Faddeev. Inverse problem of quantum scattering theory. ii. Journal of Mathematical Sciences, 5:334-396, 1976. 10.1007/BF01083780.

[7] I. M. Gel'fand and B. M. Levitan. On the determination of a differential equation from its spectral function. Izvestiya Akad. Nauk SSSR. Ser. Mat., 15:309-360, 1951. Amer. Math. Soc. Transl. (2) 1 (1955), 253-304.

[8] J. González, F. Guinea, and M. A. H. Vozmediano. The electronic spectrum of fullerenes from the Dirac equation. Nuclear Phys. B, 406(3):771-794, 1993.

[9] H. Isozaki and E. Korotayev. Inverse problems, trace formulae for discrete Schrödinger operators. ArXiv e-prints, March 2011.

[10] Hiroshi Isozaki. Inverse scattering theory for Dirac operators. Ann. Inst. H. Poincaré Phys. Théor., 66(2):237-270, 1997.

[11] Hiroshi Isozaki. Inverse spectral theory. In Topics in the theory of Schrödinger operators, pages 93-143. World Sci. Publ., River Edge, NJ, 2004.

[12] Tosio Kato and S. T. Kuroda. The abstract theory of scattering. Rocky Mountain J. Math., 1(1):127-171, 1971.

[13] Motoko Kotani, Tomoyuki Shirai, and Toshikazu Sunada. Asymptotic behavior of the transition probability of a random walk on an infinite graph. J. Funct. Anal., 159(2):664-689, 1998.

[14] Peter Kuchment and Olaf Post. On the spectra of carbon nano-structures. Comm. Math. Phys., 275(3):805-826, 2007. 
[15] S. T. Kuroda. Scattering theory for differential operators. I. Operator theory. J. Math. Soc. Japan, 25:75-104, 1973.

[16] S. T. Kuroda. Scattering theory for differential operators. II. Self-adjoint elliptic operators. J. Math. Soc. Japan, 25:222-234, 1973.

[17] V. A. Marčenko. On reconstruction of the potential energy from phases of the scattered waves. Dokl. Akad. Nauk SSSR (N.S.), 104:695-698, 1955.

[18] E. Mourre. Absence of singular continuous spectrum for certain selfadjoint operators. Comm. Math. Phys., 78(3):391-408, 1980/81.

[19] Adrian I. Nachman and Mark J. Ablowitz. A multidimensional inversescattering method. Stud. Appl. Math., 71(3):243-250, 1984.

[20] R. G. Newton. The gel'fand-levitan method in the inverse scatteering problem. In J. A. Lavita and Dordrecht J. P. Marchand, D.Reidel, editors, Scattering Theory in Mathematical Physics, 1974.

[21] R. G. Novikov and G. M. Khenkin. The $\bar{\partial}$-equation in the multidimensional inverse scattering problem. Uspekhi Mat. Nauk, 42(3(255)):93-152, 255, 1987.

[22] Michael Reed and Barry Simon. Methods of modern mathematical physics. $I V$. Analysis of operators. Academic Press [Harcourt Brace Jovanovich Publishers], New York, 1978.

[23] Michael Reed and Barry Simon. Methods of modern mathematical physics. III. Academic Press [Harcourt Brace Jovanovich Publishers], New York, 1979. Scattering theory.

[24] Gordon W. Semenoff. Condensed-matter simulation of a three-dimensional anomaly. Phys. Rev. Lett., 53(26):2449-2452, Dec 1984.

[25] Ricardo Weder. Characterization of the scattering data in multidimensional inverse scattering theory. Inverse Problems, 7(3):461-489, 1991. 